Illinois State University

ISU ReD: Research and eData

Theses and Dissertations

$10-2-2021$

\title{
Pulling Back the Carpet on the Non-Tenure Track Faculty Experience: Perceptions of Support in Postsecondary Education
}

Rachel E. Smith

Illinois State University, merachsmith@yahoo.com

Follow this and additional works at: https://ir.library.illinoisstate.edu/etd

\section{Recommended Citation}

Smith, Rachel E., "Pulling Back the Carpet on the Non-Tenure Track Faculty Experience: Perceptions of Support in Postsecondary Education" (2021). Theses and Dissertations. 1505.

https://ir.library.illinoisstate.edu/etd/1505

This Dissertation is brought to you for free and open access by ISU ReD: Research and eData. It has been accepted for inclusion in Theses and Dissertations by an authorized administrator of ISU ReD: Research and eData. For more information, please contact ISUReD@ilstu.edu. 


\section{PULLING BACK THE CARPET ON THE NON-TENURE TRACK FACULTY \\ EXPERIENCE: PERCEPTIONS OF SUPPORT \\ IN POSTSECONDARY EDUCATION}

RACHEL E. SMITH

221 Pages

This dissertation explores the perceptions of part-time and full-time non-tenure track (NTT) faculty at a Midwestern university with a focus on teaching. As NTT faculty are increasingly utilized to instruct a majority of the undergraduate courses in U.S. postsecondary education understanding their experience is essential to their integration into the institutions they represent. Little is known about the perceptions of NTTs regarding their views of preparedness and support to fulfill the expectations of their positions. This dissertation uses a qualitative grounded theory design to give voice to this new majority and in turn, provides insight to assist university campuses in developing and retaining NTT faculty. This study provides a holistic view of the experiences of both full and part-time NTT faculty from three distinct departments and includes perspectives from administrators, union representatives, and faculty developers. The findings not only affirm what is currently known in the literature but also provide new insight into the world of NTT faculty. The author argues that universities must consider a new model to support the changed professoriate, one with collaboration and support for and with both professional NTT and TT faculty.

KEYWORDS: Non-Tenure Track Faculty; Non-Tenure Track Perceptions; Professional Development; Evaluation 


\title{
PULLING BACK THE CARPET ON THE NON-TENURE TRACK FACULTY \\ EXPERIENCE: PERCEPTIONS OF SUPPORT \\ IN POSTSECONDARY EDUCATION
}

RACHEL E. SMITH

\begin{abstract}
A Dissertation Submitted in Partial Fulfillment of the Requirements for the Degree of DOCTOR OF EDUCATION

School of Teaching and Learning ILLINOIS STATE UNIVERSITY
\end{abstract}


(C) 2021 Rachel E. Smith 


\section{PULLING BACK THE CARPET ON THE NON-TENURE TRACK FACULTY \\ EXPERIENCE: PERCEPTIONS OF SUPPORT \\ IN POSTSECONDARY EDUCATION}

RACHEL E. SMITH

COMMITTEE MEMBERS:

Erin Mikulec, Chair

Tracy Mainieri

Kyle Miller 


\section{ACKNOWLEDGMENTS}

I want to acknowledge and thank my committee members for all your support and guidance throughout this journey. While I have learned so many things along the way a significant conclusion is that to be successful in education, as in life, you must surround yourself with intelligent and supportive women. I am grateful for this lesson.

I also owe a debt to my husband for making sure I ate and slept during my adventure that is a doctoral program entered into midlife during a global pandemic. Without you, I would be grossly deficient in sleep and at least 10lbs slimmer. I truly am full in more ways than one. To my children, I hope I have modeled the value of lifelong learning. These three men mean more to me than life itself. I thank the Lord for blessing me richly with them in my life. I am blessed to have amazing family, friends, coworkers, and pets that supported me along the way.

To Grandpa Casey, thank you for always believing in me. You can now call me Doctor.

R.E.S. 


\section{CONTENTS}

Page

ACKNOWLEDGMENTS

TABLES

vii

FIGURES

viii

CHAPTER I: INTRODUCTION

Background of the Study

Definition of Terms

Statement of the Problem

Purpose of the Study

Research Questions

Overview of Study

Introduction

Historical Perspectives of Faculty in Higher Education

The Non-Tenure Track Experience

Who are NTT Faculty?

Policies and Procedures

Sociopolitical Factors impacting NTT

NTT Pedagogical Preparation

Orientation, Evaluation, and NTT Professional Development

Conclusion 
$\begin{array}{ll}\text { Research Questions } & 39\end{array}$

$\begin{array}{ll}\text { Interpretivist Paradigm } & 40\end{array}$

Grounded Theory 41

$\begin{array}{lr}\text { Research Design } & 43\end{array}$

$\begin{array}{lr}\text { Participants } & 43\end{array}$

$\begin{array}{lr}\text { Data Sources } & 56\end{array}$

$\begin{array}{ll}\text { Semi-Structured Interviews } & 57\end{array}$

$\begin{array}{ll}\text { Artifacts } & 60\end{array}$

$\begin{array}{ll}\text { Memos } & 61\end{array}$

Data Analysis $\quad 63$

$\begin{array}{lr}\text { Ethics } & 68\end{array}$

$\begin{array}{ll}\text { Positionality } & 69\end{array}$

$\begin{array}{ll}\text { Trustworthiness and Limitations } & 70\end{array}$

$\begin{array}{ll}\text { Conclusion } & 71\end{array}$

CHAPTER IV: FINDINGS

$\begin{array}{ll}\text { Introduction } & 73\end{array}$

$\begin{array}{ll}\text { The NTT Network } & 74\end{array}$

Don’t They Have Orientations? 76

Lead Instructors/Program Coordinators $\quad 82$

“It's NTT Teaching NTT” $\quad 84$

Seek It Out or Be Lost $\quad 88$

"I Have a Friend" 
"It's Kind of Up to The Chemistry" 93

“So, Where My Office Is Located” 95

Identity: Things Aren’t Always What They Seem 96

The Identity of Less Than: "Just NTT” 97

"I don't really see the categories" 107

$\begin{array}{ll}\text { The Role of NTTs } & 109\end{array}$

$\begin{array}{ll}\text { Alumni: "Piece of Cake" } & 115\end{array}$

$\begin{array}{ll}\text { Essential Specialists } & 118\end{array}$

$\begin{array}{ll}\text { Representation } & 120\end{array}$

Evaluations: What's the Point? 123

$\begin{array}{ll}\text { Evaluation: Process Transcends Purpose } & 124\end{array}$

Retention: “I Really Don’t Know”

Development and Growth: Coaching for Improvement 134

Professional Development: Both A Means and An End 136

Student feedback: I Always Read It All 140

Value: It's Not All About Money 142

Inclusion: "I Just Never Felt Like a Part of The Team” 144

Stability: "I Don’t Know if I'm Gonna Have a Job” 147

Recognition: "I Just Want to Be Loved” 150

$\begin{array}{ll}\text { Better at My Job } & 151\end{array}$

$\begin{array}{ll}\text { Conclusion } & 157\end{array}$

CHAPTER V: DISCUSSION 159

$\begin{array}{ll}\text { Introduction } & 159\end{array}$ 


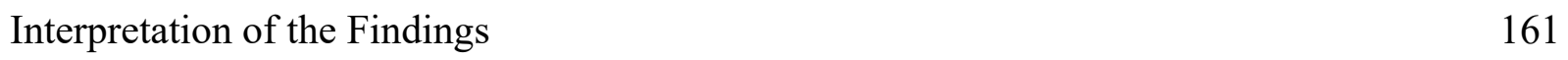

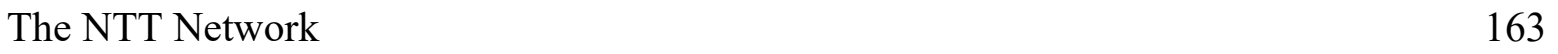

$\begin{array}{ll}\text { Identity } & 165\end{array}$

$\begin{array}{lr}\text { Evaluations } & 171\end{array}$

$\begin{array}{ll}\text { Value } & 172\end{array}$

$\begin{array}{ll}\text { Implications for Practice } & 176\end{array}$

$\begin{array}{ll}\text { Acceptance and Inclusion } & 178\end{array}$

$\begin{array}{ll}\text { Attitudes and language } & 178\end{array}$

$\begin{array}{ll}\text { Invite } & 179\end{array}$

$\begin{array}{ll}\text { Basic resources } & 180\end{array}$

$\begin{array}{ll}\text { Involvement } & 180\end{array}$

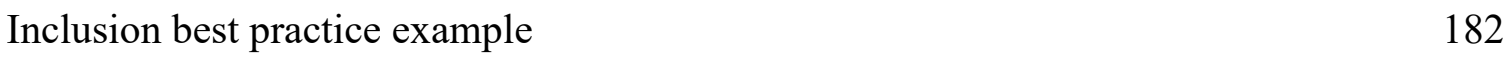

Onboarding and Professional Development 182

What alumni NTTs teach us $\quad 183$

$\begin{array}{ll}\text { Mentorship } & 184\end{array}$

$\begin{array}{ll}\text { Onboarding best practice example } & 185\end{array}$

Professional development best practice example $\quad 186$

$\begin{array}{ll}\text { Expectations and Evaluations } & 186\end{array}$

$\begin{array}{ll}\text { Explicit communication } & 187\end{array}$

$\begin{array}{ll}\text { Expectations and evaluations best practice example } & 189\end{array}$

Recommendations for Future Research 190

$\begin{array}{ll}\text { Limitations } & 191\end{array}$

$\begin{array}{ll}\text { Conclusion } & 193\end{array}$ 
REFERENCES

APPENDIX A: INTERVIEW QUESTIONS NTT FACULTY

APPENDIX B: INTERVIEW QUESTIONS UNIVERSITY EMPLOYEES

APPENDIX C: INTERVIEW QUESTIONS FACULTY DEVELOPERS

APPENDIX D: LABOR UNION REPRESENTATIVE QUESTIONS 


\section{TABLES}

Table

Page

1. Department Administrators 47

2. NTT Participants Descriptive Characteristics by Department, Full/Part-Time, Status and Gappa and Leslie Categories $\quad 48$

3. NTT Participants Alumni Status 53

4. NTT Participants Gender $\quad 54$

5. Faculty Developers 55 


\section{FIGURES}

Figure $\quad$ Page

1. Role of NTT Faculty by Department 114

2. Carpet and Floor Visual Representation Analogy Public and Hidden Transcripts 162 (“Carpet pulled back", n.d.) 


\section{CHAPTER I: INTRODUCTION \\ Background of the Study}

In the 1990's United States (U.S.) public higher education began facing budgetary cuts and decreases in state funding (Kezar, 2012). During this time, higher education began relying more heavily on Non-Tenure Track (NTT) faculty to fill the gaps in the faculty ranks caused by retirements and the economic crisis. Paired with retirements and a need to deliver coursework institutions turned to an NTT workforce to solve the problem. NTT positions cost departments less than Tenure Track (TT) lines (Zhang \& Liu, 2010). Another enticement of utilizing NTTs for teaching positions at universities is the flexibility of short-term contracts in a rapidly changing work environment (Baldwin \& Wawrzynski, 2011). NTT positions allow institutions to hire based on specialty training without the commitment and length of employment TT positions yield. Regardless of the cause, postsecondary institutions began utilizing a contingent workforce at a rapid rate.

Estimates range from $50 \%$ to $70 \%$ of faculty in U.S. higher education being categorized as NTT (Bergman, 2011; Holler, 2014; Kezar, 2014; Hensely, 2016). This is a large change in the type of educators employed at universities that once had up to $75 \%$ TT faculty (Gehrke \& Kezar, 2015). TT faculty are now the minority in higher education (Kezar, \& Maxey 2012; Sorcenelli, 2007; Champlin \& Knoedler, 2017). The model of the professoriate whereas NTT would fill in for one course temporarily and TT faculty would focus primarily on research has been flipped (Kezar \& Holcombe, 2015). The majority of undergraduate courses offered in postsecondary education are now being delivered by NTT faculty, the professoriate has changed. The increase in NTT positions is not unique to research-centered institutions. NTT faculty are being utilized for their expertise as well as efficient and economical contracts in institutions with 
teaching emphasis as well (Baldwin \& Chronister, 2001). For example, in 2009 community colleges in the U.S. employed more than 400,000 faculty; 70\% were classified as NTT. At fouryear public institutions with over 701,000 faculty; 69\% were classified as NTT faculty (Knapp, Kelly-Reid, \& Ginder, 2010). Postsecondary institutions in the U.S. vary by emphasis. Some institutions focus on research, others on liberal arts and specialized instruction, and some on general education. Public institutions offer a variety of majors and focus on a generalized education with upper-division specialization courses (Shaw, 2004). This study will focus on a large Midwestern public university with a teaching focus. R1 institutions have a research focus and often use graduate students as the contingent workforce to teach courses, thus they employ less NTT faculty (Davis, 2017). Regardless of an institution's focus, all are comprised of a heterogeneous blend of tenure-track (TT) and non-tenure-track (NTT) faculty. Regardless of the type of institution, both full and part-time NTT faculty, are used heavily to deliver undergraduate courses. This study will be limited to both full and part-time NTT positions at a four-year public institution with an emphasis on teaching undergraduate students.

\section{Definition of Terms}

Many of the terms utilized in this dissertation are common in U.S. higher education. However, assuming the meanings of terms can be a hindrance in communication. Especially, considering over 50 different categorical labels can be found that describe NTT employment (Berry, 2005; Kezar, 2012). For example, adjunct, contingent, part-time, interim, and temporary are all terms used to describe employees hired to teach on a course-by-course basis. Much of the literature uses these terms interchangeably. Inconsistent terminology in the literature and common usage can be confusing. It is therefore important to define terms in this study to provide clarity for the reader. 
Postsecondary Education. Postsecondary education and higher education will be used interchangeably to mean schooling beyond high school. These broad terms include both two-year community colleges as well as four-year universities. This study focuses on a four-year postsecondary university whose primary focus is teaching with a secondary emphasis on research and service.

Faculty. The employees hired by an institution of postsecondary education to impart knowledge to students, regardless of the employees' tenure status. This paper defines faculty as a body of educators in postsecondary education inclusive of both TT and NTT employees. See also professoriate.

Tenure-Track Faculty. Tenure Track Faculty (TT) faculty are employees in higher education characterized by a threefold expectation of teaching, research, and service. The trajectory for advancement in these positions is a tiered ladder system of promotional expectations that varies with the type of institution (Bland, Center, Finstad, Risbey, \& Staples, 2006). Postsecondary institutions are categorized by their research focus; those with the most intense research focus are categorized as research one (R1) meaning research is the number one priority at this institution. The continuum continues with research two (R2) having a moderate focus on research and concluding with research three (R3) indicating schools with a moderate focus on research and a high emphasis on teaching. This classification system was determined by the Carnegie Commission on Higher Education in 1971 to support research in higher education by providing clarity in terms (McCormick, 2001). Universities have complex systems of promotion and retention for TT faculty based on their ability to perform in the three areas of research, teaching, and service. This study will define TT faculty as those with full-time appointments that advance 
on a track that can culminate in a tenured position within the institution. Tenured faculty usually hold the ranks of full, associate, and assistant professors.

Non-Tenure-Track Faculty. In postsecondary education, non-tenure-track (NTT), and sometimes referred to as adjunct or contingent, positions are hired primarily to teach. NTT positions provide instruction in general education and teach the bulk of lower-division undergraduate courses (Scott \& Danley-Scott, 2015). This paper will use the term non-tenure track to describe faculty hired specifically to deliver courses on a limited-term inclusive of both full and part-time appointments. Graduate students hold a separate category of adjuncts in their expectations to gain teaching experience and earn tuition waivers as well as other benefits and thus are being excluded from the definition of NTT in this paper. Additionally, NTT hired to be administrators or as researchers such as is common in R1 institutions are excluded from this definition for this study. NTT is the term that is the most recognizable in the literature and includes both part-time and full-time employees whose roles are primarily teaching (Kezar \& Sam, 2010).

Career Ender. A term used by Gappa and Leslie (1993) to categorize NTT faculty who are retired and teaching courses to fill in on a temporary basis. These NTT do not intend on working full-time.

Specialist. A term used by Gappa and Leslie (1993) to describe NTT with discipline-specific expert knowledge. These individuals may teach full-time or have a career in a related profession and teach contingently.

Freelancer. A term used by Gappa and Leslie (1993) to describe NTT faculty that choose to teach part-time for the benefit of having a flexible schedule. 
Aspiring academic. A term used by Gappa and Leslie (1993) to categorize NTT faculty that are in search of full-time academic appointments. These NTT may hold terminal degrees and may teach courses at multiple institutions to piece together a wage-earning job.

Professoriate. A term used to define educators collectively in postsecondary education to impart knowledge to students, regardless of the employees' tenure status. Thus, the terms faculty and professoriate are defined for this paper as the body of educators of an institution of postsecondary education inclusive of both TT and NTT employees and will be used interchangeably. See also, faculty.

Professional Development. Programs designed to improve upon a faculty member's instructional skill are commonly referred to as faculty development. Many universities have centers, departments, or offices dedicated to the cause of acclimating and training faculty for the various aspects of their positions. University centers for teaching and learning have been charged with a more holistic view of development to address the needs of faculty work including not only teaching but areas such as organization, research, and citizenship (Amundsen et al., 2005). This study will use the term professional development to encompass both faculty development specific to pedagogy as well as a more holistic offering of resources to expand a faculty member's skill needed in higher education. This would include not only programs, but also texts, mentors, online resources, and other organizational supports. The work of de Saxe Zerden, Ilinitch, Carlston, Knutson, Blesdoe, and Howard (2015) emphasizes the term professional development to explain the skills necessary for NTT faculty to complete their complex roles as educators in all aspects of their professional lives.

Faculty Developers. Faculty developers are the individuals charged with organizing courses, centers, and workshops to meet the continuing educational needs of the faculty. Individuals often 
work in offices titled centers for teaching and learning, faculty improvement, or academic development. The term academic development often includes programmatic offerings such as orientations, life balance, and other foci in addition to pedagogy (Amundsen et al., 2005). However, the literature frequently does not distinguish between the terms academic and faculty development, thus they are used interchangeably.

Pedagogical Preparation. The term pedagogy refers to skill in teaching methods (Hativa, Barak \& Simhi, 2001). Pedagogical preparation includes coursework, training, independent study, readings, and other tasks that faculty pursue for the purpose of improving or informing their teaching practice.

\section{Statement of the Problem}

Given postsecondary education's reliance on NTT faculty to deliver courses it is worthy to evaluate their perceived preparedness as educators. With such a large number of NTTs teaching, are they prepared to perform all aspects of their positions? Further, how can the institution fully support the pedagogical development of NTT faculty? Much of the discussion in the literature regarding NTT faculty focuses on pay equity, working conditions, job protection, and the exploration of how the inversion of the professoriate occurred with little focus on the experiences of NTT faculty themselves (Feldman \& Turnley, 2001; Fuller, Brown, \& Smith, 2017). With such a large number of NTT instructors fulfilling the role of teaching in higher education, research on their perceptions and pedagogical preparation is lacking. NTT faculty have become essential to the educational institutions in which they work but are often treated as outsiders, not given resources and support to succeed (Davis, 2017; Hensely, 2016; Hoeller, 2014). Additionally, NTTs are often do not receive training to do all aspects of their positions yet are retained based on evaluations as if they should have this knowledge. Exploring the 
opinions and perceptions of NTT faculty regarding their teaching and preparation is valuable in understanding the NTT experience and in turn assisting in the development of programs to meet the needs of this eclectic group.

NTTs are not required to have terminal degrees as a minimum qualification of employment. However, many scholars agree that doctoral education simply does not adequately prepare faculty to teach (Adams, 2002; Pace, 2017; Schmid et al., 2016). NTTs vary greatly in the level of formal pedagogical preparation from none to in-class observations, to graduate teaching assistant positions, and terminal pedagogical degrees (Fuller, Brown, \& Smith, 2017). Institutions seeing the gap in training for faculty as a whole have developed centers for continuing professional development. These departments offer training that motivated faculty can attend on topics such as classroom skills, working with diverse learners, and assessment of learning (Eddy, 2010). However, much of this learning is self-directed and optional with more intrinsic than extrinsic motivation. Without formal training, we teach in ways that reflect our character, values, and our own past experiences with teaching (Weimer, 2004). Faculty draw upon knowledge as well as prior experience when teaching and could benefit from on-the-job training Olesen and Hora (2013). Formalizing the process in which faculty are orientated and evaluated could assist not only the NTT employee but also the administration and faculty developers.

Faculty developers charged with equipping professors in pedagogical development throughout their careers as well as position preparation and orientation when faculty are first hired may benefit from a better understanding of the unique needs of this new majority. Information from the NTT faculty themselves on the level of pedagogical preparation they have upon hire and what supports are perceived as needed would provide direction to target training 
and experiences to best meet the needs. For example, McClenny and Arnsparger (2014) found that NTT faculty in community colleges have fewer opportunities to participate in professional development, however, if given the appropriate supports NTTs could increase their skill at teaching. Exploring what specific supports are wanted and/or needed for NTTs at four-year teaching-focused institutions has implications not only for the NTT faculty but also for their students.

Additionally, insight into how NTT faculty are evaluated and utilize these evaluations to inform teaching adds to the current limited scholarly work in this area. UW-Madison's early career faculty professional development programming is an example of a best practice model for faculty pedagogical development. In this program, first-year faculty are introduced to methods of instruction and assessment to infuse into their teaching practice (Schmid et. al, 2016). These programs however focus on TT faculty and do not meet the unique and diverse needs that are a part of the NTT experience.

Most professions provide some type of job training to ensure job tasks are understood and completed in a quality manner, this is not always the case with NTT faculty. Kezar (2013b) interviewed 107 NTTs in 25 departments from three different institutions and found evaluations and orientations varied widely from none being offered at all to extensive comprehensive and collaborative programs. Universities are relying heavily on a workforce to educate undergraduate students without intimate knowledge of the supports needed for those employees to fulfill the expectations of their positions. In summary, there are a large number of NTTs, they are often given limited to no training in preparation for the complexity of their positions yet are retained based on evaluations as if they should have this knowledge. 


\section{Purpose of the Study}

This study strives to give voice to the NTT faculty in higher education at a large, fouryear Midwestern teaching-focused state university. The emphasis of the study will be to examine the perceptions of NTTs in terms of their feelings regarding pedagogical preparation to teach at the postsecondary level and the support they receive or do not receive, to do this effectively. The goal of the study is intended to make recommendations for potential future pedagogical supports and affirm those methods which are currently perceived as beneficial by the NTT faculty. Identifying perceived faculty preparation has implications not only to the NTT employee, but also to faculty developers, and administrative stakeholders that strive to provide ongoing support and keep up with hiring trends in postsecondary education.

\section{Research Questions}

This study will explore NTT faculty opinions and perceptions of their experiences at a Midwestern state teaching university. The following questions will be addressed:

RQ 1: How pedagogically prepared did NTTs feel when they began teaching at the postsecondary level?

1a. What types of previous experiences or pedagogical training inform NTT perceptions of preparedness?

RQ 2. How do NTT faculty describe the pedagogical support they receive in their teaching roles?

2a. What is the role of professional development in supporting NTT faculty teaching?

2b. How are evaluations used to inform NTT faculty teaching and retention?

2c. What additional supports do NTT faculty desire to improve their teaching? 


\section{Overview of Study}

Chapter I explained changes in the professoriate in postsecondary education. This chapter established research questions and defined terms that will be utilized throughout the study as well as explained the problem and purpose of the study.

Chapter II outlines the history and changing nature of the professoriate to provide context. This chapter examines the current empirical research and literature concerning the NTT faculty teaching experience, professional development, and teaching evaluations. Further, the role of professional development is explained in a postsecondary context. Lastly, the theoretical framework which informs the study will be discussed.

Chapter III outlines the research design for this project. This study uses predetermined descriptive categories to describe the sample and further explore the data based on NTT participant characteristics. This chapter describes the qualitative semi-structured interview methodology used. Interviews were conducted to gain firsthand perceptions of the subjects. Grounded Theory methodology was used to analyze data. Issues of trustworthiness, triangulation, member checking, and bias are included along with the limitations of the study.

Chapter IV presents the results of the study. This includes understandings from interviews and artifacts. Interviews with NTT serve as the primary datum. Interviews with faculty developers, administrators, union representatives, and corresponding documents serve to aid in interpreting the context of the data.

Chapter V presents the conclusions which can be drawn from the research and interpretations in relation to the current literature. The chapter also suggests areas of future research and discusses limitations. 


\section{CHAPTER II: REVIEW OF THE LITERATURE}

\section{Introduction}

This chapter will synthesize the existing literature on the NTT experience, the importance of evaluation to inform teaching practice, and the role of professional development in providing training opportunities. The chapter begins with a historical explanation of the change in higher education from a tenured system to an NTT model to provide context. Historical contextualization describes the current state of the professoriate in postsecondary education, the environment in which NTT faculty are immersed, and broadly the need for this study. Next, the chapter explores the NTT experience including responsibilities in the areas of procedural knowledge, pedagogical preparation, and sociopolitical factors. This section will explore the relevant research that provides insight into what is common among NTTs in higher education. Exploring the varied expectations of NTTs helps to create a picture of the day-to-day expectations as well as the tacit cultural assumptions involved in being an NTT faculty member. These tacit assumptions are dynamic and are impacted by the intersections of complex issues such as discipline area, cultural assumptions, and sociopolitical factors. The sociopolitical factors along with procedural and pedagogical preparation have implications for NTTs in the area of evaluation.

Evaluation and its impact on the NTT experience will be discussed through a review of the recent literature. Evaluation will be explored specifically for its relevance in informing teaching practice for NTTs, but also as a form of assessment for retention and rehire. The use of evaluation of NTTs is complex, varied, and holds nuanced implicit and explicit implications for the NTT faculty member. 
Once the NTTs evaluation experiences have been discussed, the literature review will turn to investigate the role of professional development in higher education. Understanding professional development and the potential impact on the preparation of NTTs will serve as background to position the research project to have direct applicability for postsecondary institutions. The foundation of the study begins with the historical context in which NTTs emerged as a majority faculty type in higher education.

\section{Historical Perspectives of Faculty in Higher Education}

Educators in U.S. postsecondary education are a demographically and professionally complex and varied group (Kezar \& Sam, 2010). This section will provide a brief background of the professoriate to aid in understanding the formations of this eclectic group. In explaining organizational culture Schein (2016) emphasizes a group's shared history as impactful in understanding the current context. History will help frame the change in higher education from a traditional tenured system to an NTT model relying heavily on the employment of NTT faculty to deliver undergraduate courses. This context provides background for the study.

The standard view of a university professor is one of respect, security, and status (Champlin \& Knoedler, 2017). TT faculty members have work expectations of research, teaching, and service that comprise the traditional TT model (Kezar, 2013; Finkelstein \& Schuster, 2011). However, this has not always been the case. Thelin (2011) notes the history of TT faculty in the U.S. lies in tutoring and moral education. Clergy often held these temporary positions while awaiting appointments to churches (Kezar \& Maxey, 2016). According to Thelin (2011), the original colonial colleges were founded by Puritans and thus the work of the faculty was essentially moral Christian education intended to ensure proper social and religious etiquette for the elite who would inherit finances, businesses, and political positions. Postsecondary 
education began as a glorified finishing school. The university changed with the culture and over time established specific work expectations for faculty. Between 1890 and 1910 the professoriate became associated with holding discipline-specific content knowledge (Thelin, 2011). Having such knowledge gained from specific research transformed the role of the faculty to one holding higher prestige. Essentially faculty became content experts. Research was tied with this expertise which led to faculty job security.

Gradation of ranks tied with specific universities for employment security through a tenure and promotion process and academic freedom were all formalized in the early 1900s (Thelin, 2011). These concepts began to formalize the role of the professor. The American Association of University Professors (AAUP) was founded in 1915 to protect the professoriate and preserve academic freedom (History AAUP). The AAUP introduced the ideas of faculty governance for faculty to have a role in decisions made by the university and to protect faculty who may teach or research controversial subjects. The traditional tenure model of teaching, research, and service was born out of the formalization of the professoriate. The introduction of the GI Bill required that the traditional model be reconsidered. It is at this point in the history of the professoriate that the NTT faculty roles were introduced deviating from the traditional tenure model.

Officially entitled the Servicemen's Readjustment Act of 1944, the GI bill provided educational benefits based on military service. Many universities doubled their enrollment with returning soldiers and diverse students as a result of the Civil Rights movement (Thelin, 2011; Kezar, 2014). The increase in enrollment necessitated an expansion of the professoriate. According to Bland et al (2006) NTT appointments were used to supplement the faculty ranks with an increase in full-time NTT hires between 1975 and 1993 of 88\%, while TT decreased 9\% 
for the same period. Federal legislation in the 1970s and 1980s had a direct impact on the retirement age of TT faculty, causing a rise in incentive base retirement programs for TT faculty which also contributed to the increase in NTT faculty hires (Baldwin \& Chronister, 2001). Postsecondary education faced large budget cuts and faculty retirements in the 1990s which continued the reliance on NTT positions (Kezar, 2012). Several authors have sought to find the cause for the increase in NTT and decrease in TT appointments.

The literature provides several options in seeking the cause of the increase in NTT faculty including financial, flexible, specialized training, and fluctuations in enrollment. Zhang \& Liu (2010) believe financial motivations prevailed as NTT appointments cost departments less money than TT lines. Baldwin and Chronister (2002) agree with the economic motivation and add flexibility in hiring as a motivation. Short-term contracts allow for the hiring of expertise and specialized talent without a commitment to long-term employment (Baldwin \& Wawrzynski, 2011). Flexible hiring allows for universities to select practitioners, retirees, or those with specialty certifications such as a Certified Public Accountant (CPA) in an Accounting Department to teach with real-world experience. Often hiring an NTT retiree frees up TT faculty to focus on research of other projects. Kezar \& Gehrke (2014) add the inability to have TT positions approved and increased enrollment to the list of forces that drove the NTT hiring increases. The lack of approval in hiring is typically financially motivated but may also be related to university politics. One such political decision relates to increases in student enrollment. Increased enrollments are often due to market changes or shifts in demands for specific majors and fluctuate greatly (Kezar, 2014). Hiring NTT faculty allows for last-minute decisions in educators to cover courses when enrollment is up. Conversely, when enrollment has waned in a major, the NTT position is not continued for the subsequent year or semester. Thus, 
flexibility to supplement the teaching load with NTT faculty, without going through the lengthy process to hire a TT position appeals to administrators. Regardless of the cause, postsecondary institutions began utilizing a contingent workforce at a rapid rate.

NTT faculty are the new faculty majority accounting for $50-70 \%$ of the professoriate in U.S. postsecondary institutions (Bergman, 2011; Holler, 2014; Kezar, 2014; Hensely, 2016). This trend of increase in NTT faculty appointments alongside a decrease in TT did not go unnoticed, however, finding specific and current data on both full and part-time NTT groups proves difficult. The National Center for Education Statistics conducted the National Study of Postsecondary Faculty (NSOPF) sporadically in 1988, 1993, 1999, and 2004, to provide a profile of all postsecondary faculty (National Center for Education Statistics, 2018). This comprehensive survey was intended to provide demographic statistics, professional background information, and data on workload and job satisfaction in higher education (National Center for Education Statistics, 2018). The survey spotted the trend in the increase of NTT positions; however, it had had very limited results specific to NTT faculty positions in detail thus giving only a limited view of the faculty as a whole.

The survey included public, private, not-for-profit two and four-year postsecondary institutions in the U.S with accreditation by the U.S. Department of Education, making it extremely comprehensive. Additionally, the survey had a control from the Integrated Postsecondary Education Data System (IPEDS) faculty survey conducted annually also by the National Center for Education Statistics (National Center for Education Statistics, 2018). This control allowed researchers to account for any discrepancy in the data. Researchers did note a discrepancy in how faculty numbers were reported. For example, some universities provided full-time equivalent data rather than actual headcount. Another error is found in the reporting of 
the number of part-time faculty, typically considered NTT. This group was often misreported due to confusion of the definition of appointment type. This would mean the numbers of faculty in the study overall would be over or underrepresented. Considering, IPEDS and NSOPF have differing definitions of faculty positions, with IPEDS classifying based on primary responsibility and NSOPF categories based on any responsibility it becomes even more difficult to distinguish which faculty were truly in TT versus NTT roles at the time of survey (National Center for Education Statistics, 2018). This is a common frustration in research regarding NTT positions.

The literature often makes no distinctions between NTT positions teaching one course sporadically versus full-time career NTT or retirees, making it difficult to draw conclusions in both the number of positions as well as explore the differences in the NTT experiences by appointment type. Cross \& Goldenberg (2009) note another complication in studies on NTT is a lack of uniformity in terms used to classify NTT. Thus, literature uses a blend of terms including contingent, adjunct, non-tenure track, lecturer, instructor, temporary, and many others. As the NOSPF is no longer being funded nor conducted the University of California, Los Angeles Higher Education Research Institute (HERI) faculty survey conducted every three years including 20,771 full-time undergraduate faculty from 143 baccalaureate institutions becomes a valuable source (Stolzenberg et al, 2017). The HERI focuses on full-time undergraduate teaching faculty, however, this study excludes part-time NTTs. The survey included demographic information as well as how faculty spend their time, preferred methods of teaching, and perception of campus climate and topics such as stressors (Stolzenberg et al, 2017). The study does provide some insights on the professoriate, of which NTTs are a part. Specifically, the study serves as a profile pointing out the following: racial discrimination as a source of stress, faculty roles in developing critical thinking skills among students, and the abundance of 
NTT teaching remedial courses as well as professional development (Stolzenberg et al, 2017). However, this study is limited in specific information to the NTT experience as it is quantitative in nature which does not allow for the in-depth narratives possible through qualitative research. Additionally, the full results are available only to those who purchase the data, limiting its usefulness and availability for novice researchers.

Lacking a readily available national survey requires researchers to rely upon individual studies to assist in understanding the demographic characteristics of the professoriate. Arguably the most extensive source of information on the characteristics of the professoriate is the work of Kezar (2018), who has coordinated extensive research including a Delphi project on changing faculty and student success. This project surveyed faculty, deans, provosts, and policymakers to determine the future of the blended professoriate. The Delphi contributes to what is known about the professoriate, but also provides insights specific to NTTs. The study includes information that NTT faculty including both part and full-time make up to $70 \%$ of all faculty in nonprofit postsecondary institutions (Kezar \& Maxey, 2015). The research of Kezar and Maxey (2015) explores a changing professoriate caused by a variety of factors that resulted in a haphazard and scattered response to staffing issues in higher education. The goal of the Delphi was to begin conversations of alternative models to the TT/NTT system that exists today. Kezar and Maxey (2015) outline historical changes that led to a majority NTT model as well as problems that have arisen out of the existing model, but ultimately turn to the future of faculty roles in higher education. NTTs fill about 70\% of instructional positions nationwide (Brown Barnes, 2017). Since it is estimated that three out of every four new faculty hires are an NTT the traditional TT system is no longer exists in U.S. higher education (Kezar \& Gehrke, 2014). 
As suggested by Champlin and Knoedler (2017) the traditional view of the esteemed TT professor juggling research, teaching and service now represent the minority of faculty in higher education. Regardless of the cause of the increase in NTT faculty, this new majority is a complex group to study due to a lack of consistency in definitions and nonuniformity in government reporting methods. The new majority of the professoriate may or may not be engaged in research and service, however, they are teaching the bulk of undergraduate courses in postsecondary higher education. The steady employment of NTT positions both part and full-time shows no signs of decreasing. Providing information on the inversion of the professoriate and the lack of qualitative information available from national studies affirms the need for the current research to contribute to what can be known about the new faculty majority. In an attempt to better understand the characteristics of this new majority, the next section will synthesize the current literature on the NTT experience.

\section{The Non-Tenure Track Experience}

This section will explore the experience of being in an NTT faculty position in U.S. postsecondary education, including the areas of procedural knowledge, pedagogical preparation, and sociopolitical factors. This section will discuss the unique aspects of holding an NTT appointment and explore differences and similarities that are currently known among this diverse group. This section will seek to explain what is currently known about the NTT faculty experience to position the proposed study to fill the gap in what is currently known about the NTT experience.

\section{Who are NTT Faculty?}

The literature uses over 50 different terms to describe faculty hired specifically to teach undergraduate courses on a limited term including both those hired part and full-time (Kezar, 
2012). As noted, a complication in studies on NTTs is a lack of uniformity in terms used to classify NTTs (Cross \& Goldenberg, 2009). This makes it a challenge to parse out differences between part-time and full-time NTTs as some studies lump both groups together. These studies make no distinction between the NTTs hired to fill in for a retirement one semester versus the career NTTs who choose not to pursue a terminal degree or the NTTs hoping to gain experience to market themselves into a TT line. The individuals described would have vastly different motivations, backgrounds, and needs concerning preparation, development, and evaluation. Additionally, there is no current standard policy in postsecondary education in reporting the data of appointment types within the professoriate (Fuller, Brown, \& Smith, 2017). Further individual research studies establish their own methodology and definitions and may choose to classify adjunct, temporary and full-time NTTs as one position type. To be fair, this study also has a working definition of NTT as faculty hired specifically to deliver courses on a limited term, regardless of the number of courses taught. However, the experience of the NTTs may vary based on the length of their contract. As Davis (2017) points out a challenge in the literature is the assumption that all contingency is the same. This assumption perpetuates misunderstanding among the quality and image of the NTT employee.

As early as 1978 with the work of H.P. Tuckman researchers struggled to understand NTT workers. Tuckman proposed four main categories of part-time professors with unique characteristics including Semi-retired, Graduate students, Hopeful full-timers, Fullmooners, Partmooners, and Part Unknowners (Tuckman, 1978). In their seminal work, Gappa and Leslie (1993) expanded upon Tuckman's work and separated NTTs into the following subcategories: Career Enders, Specialists, Freelancers, and Aspiring Academics. This stratification of NTTs helps to distinguish some differences in the working conditions and clusters the Tuckman 
categories into manageable groupings. Career Enders are retirees teaching courses to fill in on a temporary basis, not intended on working full-time. Specialists are NTTs with discipline-specific expert knowledge. These individuals may teach full-time or have a career in a related profession and teach contingently. Freelancers choose to teach part-time for the benefit of flexible schedules. Lastly, Aspiring Academics are actively in search of full-time academic appointments. These NTTs may hold terminal degrees and may teach courses at multiple institutions to piece together a wage-earning job (Davis, 2017; Gappa \& Leslie, 1993). NTTs have grown rapidly and represent a diverse group with differing motivations for employment. Muncaster (2011) sought to update the Gappa and Leslie findings by adding two additional categories: Road Scholars and Minimalists. The additional category of Road Scholars describes individuals who teach for many institutions at one time while the term Minimalists is used for those that teach only two courses a year, are not retired, and have no aspirations for further employment in or outside of higher education. These supplementary categories have appeal in further supporting the distinctions in NTT faculty positions in recent years, however further research is needed to include these into the accepted literature. It is of note that recent news exposing part-time NTTs working at multiple institutions and living near the poverty line would be in support of Muncaster's Road Scholar classification. However, it could also be argued that these individuals fall into the Aspiring Academic category. Additionally, with the increase of online course delivery, a new category of Digital Scholars may be warranted. Fuller, Brown, and Smith (2017) support the conclusion to utilize Gappa and Leslie's classifications but suggest additional distinction is needed in the categories to accommodate the inherent differences between full and part-time NTTs. A clear labeling system helps to classify and communicate information about this heterogeneous and growing group. Ultimately the work of Gappa and 
Leslie is not only clear and accurate but also most widely accepted and will be utilized in this study as well. These classifications will serve as a springboard to help study how participants frame their role and employment experience. Using Gappa and Leslie's framework will bring consistency to a topic that is often disjointed.

The government watchdogs, the United States Government Accountability Office (GAO), noted NTTs play a large role in postsecondary education. The GAO report (Brown Barnes, 2017) summarizes what is known about both full-time and part-time NTTs from 9 postsecondary institutions in Georgia, North Dakota, and Ohio and includes information on the roles of NTTs, the economic circumstances of NTTs employment, and advantages/disadvantages of this type of work. Through administrator interviews and 21 NTT faculty discussion groups Brown Barnes, (2017) found disadvantages in the NTT positions such as heavy teaching loads with low compensation and often no health or retirement benefits as well as job instability, limited career advancement, limited input into institutional decision making, and lack of institutional support. Next, the report listed several advantages of NTT employment such as flexibility of schedules, ability to focus on teaching, opportunity to work with students. It is important to note that all campuses have different working conditions and attempting to generalize or make assumptions that all NTTs have the same experience would be too simplistic. However, without information on this large and heterogeneous group, it is difficult to meet their needs. In an attempt to better understand factors that influence the NTT experience, it will be broken into three areas: policies and procedures, sociopolitical factors, and pedagogical preparation. While these three areas are not exhaustive to the NTT experience, they do represent the trajectory of the current literature regarding NTT faculty. 


\section{Policies and Procedures}

Where to park, how to make photocopies, when to order textbooks, guidelines on office hours, how to order office supplies, scheduling of course times, and entering grades into course management systems are all tasks that can be considered procedural knowledge related to NTT faculty work. This type of knowledge is impacted by the institution and departmental policies. For example, in compliance with the Americans with Disabilities Act, all U.S. postsecondary institutions have policies related to academic accommodations for students with disabilities. However, the procedure varies from campus to campus in how students self-disclose, what offices respond, and in turn how faculty are made aware of accommodations. Policy and procedure are intertwined information that is often conveyed to new employees during orientations. However, it has been well documented that both part-time and full-time NTTs may receive no formalized orientation and are often lacking resources to complete procedural tasks (Hart, 2011; Kezar \& Sam, 2010; Ott \& Cisneros, 2015). A lack of resources and knowledge is reported by full-time and part-time NTTs alike. However, Ott and Cisneros (2015) found that full-time NTTs desired a more professionalized work environment consistent with their continuous employment status.

Kezar (2013a, 2013b) notes that both policy and resources affect NTT faculty performance. In using HERI data to evaluate policies and practices that impact part-time NTTs job satisfaction, Eagan, Jaeger, and Grantham (2015) found that procedural supports such as computers and office space are important to part-time NTTs and aid in feelings of respect and professionalism. Having adequate working conditions, being valued for teaching contributions, and having input into policies through faculty governance would serve to integrate NTTs into the campus culture (Kezar, 2012). 
Both part and full-time NTTs are limited in their involvement with decisions on campus, often referred to as shared governance. Olson (2009) explains shared governance as collaborative communication. This does not necessarily mean every campus decision is voted upon by all constituents. On the contrary, shared governance is an opportunity to contribute and be a part of the decision-making process at different levels within the university. Faculty become a part of governance by being a part of decisions related to curriculum and policies impacting the academics of the institution. This involvement in turn leads to a sense of belonging, value, and contribution to the university as a whole (Kezar,2012). There is very little research on the role of NTTs in university decision-making. Gappa and Leslie (1993) found that within the 18 institutions they studied, part-time NTTs have a desire to be more involved in governance, but that actual involvement is minimal. Baldwin and Chronister (2001) evaluated full-time NTTs and found varying levels of involvement in committees and governance, but again a desire by the full-time NTTs to be more involved with their institutions in this manner. Part-time NTTs are often excluded from involvement in governance including attendance at departmental meetings (Baldwin \& Chronister, 2001; Gappa \& Leslie, 1993). Lack of involvement has several possible motivations including the speculation that NTTs do not want to be involved due to lack of connectivity with the institution, time constraints juggling multiple jobs, and fear of losing their teaching positions if committees they serve on make decisions that are viewed unfavorably by the administration (Morrison, 2008). Other reasons NTT faculty would want to be involved but do not participate include exclusion by university policy, such as having representation in much lower percentages than TT, and the social exclusion brought on by feeling undervalued (Gehrke \& Kezar, 2015; Morrison, 2008). Part-time NTTs are often denied participation in faculty governance (Schmidt, 2013). However, in a recent study, 115 doctoral-granting institutions' 
web-based artifacts were reviewed by Jones, Hutchens, Hulbert, Lewis, and Brown (2017) and found that full-time NTTs are often included in faculty governance through representation on Faculty Senate. It would appear that in the gap of time between these studies full-time NTTs have been included at least in Faculty Senate at the institutions studied. This speaks to doctoral institutions adapting to the new current majority. Jones et al. (2017) did note that the policies reviewed were ambiguous and thus evaluating the practice on campus would be needed to determine if NTTs faculty are actually participating in governance. The only determination made was that full-time faculty could participate through having a seat available on Faculty Senate. Alleman and Haviland (2016) found that NTTs felt limited agency and respect in the workplace and ultimately want to have a voice in campus decisions at multiple levels. NTT faculty may have a seat at the table but not feel welcome to sit down.

Kezar (2013b) interviewed over 100 part-time and full-time NTTs regarding their perception of departmental and university policies and how these policies impacted their performance, noting when participants brought up policies as having a negative or positive impact on their performance. Kezar found negative policies impacting NTTs included: "scheduling classes, lack of curriculum input, learning resources, feedback, and lack of learning infrastructures" (Kezar, 2013b, p.582), and positive policies were the same categories in the reverse. The negative policies were found more often with part-time NTTs. For example, NTTs may not know what they are teaching until weeks or days before the beginning of the academic term. Gappa and Leslie (1993) note incidents of course assignments mere days before the start of the term leaving limited time for preparation. This is an example of how procedure impacts pedagogy. Not knowing what course topic will be taught impacts one's ability to prepare any materials in advance. A further example of exclusion in procedural knowledge impacting 
performance is many NTTs reveal they do not have input into textbook selection (Kezar, 2013b).

As the text selection in undergraduate coursework is often tied with the course development, exclusion in this procedure also limits pedagogical preparation. The seemingly insignificant omissions in the procedure may have a long-lasting impact on pedagogical preparation and in turn on NTT faculty evaluations. The NTT instructor may appear to the students to be unprepared which could be due to a late hiring process and not necessarily a lack of ability or organization. These examples demonstrate a larger phenomenon of how including or excluding NTTs in procedures and policies has an impact on their productivity. While tasks such as selecting textbooks and scheduling preferences are explicit components of the NTT experience some factors are more implicit, these include sociopolitical factors.

\section{Sociopolitical Factors impacting NTT}

Every workplace has nuances that are implied behavioral expectations and social norms. The tacit assumptions in the NTT faculty environment go beyond cultural implications. Culture is defined as the knowledge, beliefs, customs, and habits of groups (Schein, 2016). While universities, departments, and groups of employees certainly develop work culture, the term does not fully represent the tensions present in a politically charged environment. Instead, the term sociopolitical will be used to embrace the meaning of a system that has both sociocultural and political aspects. This term more fully encompasses postsecondary higher education. Bolman and Deal (2013) point out that organizations that have limited resources and distinct power structures may best be characterized through a political framework rather than merely focusing on culture. Postsecondary institutions with diminished funding employ higher numbers of NTTs (Davis, 2017). This alone would fit the definition provided by Bolman and Deal, however, universities also have clearly defined power structures regarding faculty governance, policy, and procedure. 
The sociopolitical environment in which the new NTT faculty enters higher education is one with tensions of unclear workplace expectations and ambiguous identities.

Mehta (2013) astutely states that how problems are framed impacts not only the solutions pursued but also the power distribution of who has a voice in solving the problem. The perceived problem of an increase in NTTs was researched primarily by TT faculty and framed as a deficit model. Research regarding NTT faculty began in this deficit model, trying to determine the number, the cause of the increase, and the competency of NTT faculty. In 2007 Umbach asked the question, "How Effective Are They?", this seminal work epitomizes the sociopolitical factors impacting the NTT experience. Citied in countless literature reviews, Umbach concludes that NTT faculty are less effective educators than their TT counterparts (Umbach, 2007). TT researchers became concerned with the increase in NTT faculty and embraced the deficiency framework provided by Umbach. Being concerned that TT employment was in danger due to the increase in NTT hires, studies began to explore the causes of the increase in NTTs (Champlin \& Knoedler, 2017). This line of research added to an already separatist culture.

A hierarchy of advancement exists in postsecondary institutions for TT faculty. When supplementing with peripheral NTTs, these positions often fall at the bottom of the sociopolitical runs of the ladder. Indeed Umbach's (2007) choice of 'us' and 'them' terminology demonstrates the 'othering' of NTT's. An important clarification in Umbach's findings is that while he concluded part-time NTTs were less effective than TTs in teaching undergraduate courses, fulltime NTTs were found to be equally as effective as TT faculty. Furthermore, as both Umbach (2007) and Champlin and Knoedler (2017) point out, the differences found in teaching in this study may be directly related to adverse working conditions, lack of professional development, and a failure of postsecondary institutions to acclimate NTTs to the expectations of their 
positions. Kezar and Maxey (2013) also point to the job structure as the deficit model impacting NTT's rather than a lack of ability.

The dualistic system of TT and NTT faculty is apparent both in practice and in literature. Ott and Cisneros (2015, p.16) summarize the sentiment best in their statement, "NTT faculty are an overlooked population both as individuals on campus and collectively in the scholarship." While it is not always the case at every university, often NTTs are not regarded as equal members of the faculty ranks within the framework of the university. NTTs report their work experience as being left out or feeling like second-class citizens (Davis, 2017; Gappa \& Leslie, 1993; Kezar, 2012). Hart (2011) found that a lack of resources contributed to a sense of alienation. Supportive and collegial workspaces can add a sense of job satisfaction for all faculty regardless of appointment type (Gappa \& Leslie, 1993; Merrit Boyd, 2016). The sociopolitical factors that intermingle in postsecondary education create a tightrope environment where NTT faculty are desired for their ability and willingness to teach on short notice for lower wages, but not fully embraced and supported by the system itself. This is not necessarily due to negative perceptions from TT faculty, but the systemic barriers created by a two-part system of us and them. Ott and Cisneros (2015) quantitative study of full-time NTT faculty confirms that NTT faculty have perceived lower levels of fair treatment in the workplace and both personal and professional satisfaction compared to their TT counterparts. NTT faculty attribute this directly to lack of participation in campus governance, lack of social inclusion less procedural support, and few teaching resources (Kezar, 2012; Ott \& Cisneros, 2015). The NTT experience can be summarized as classed and influenced directly by administrative preference of favor or indifference (Hart (2011). Postsecondary administrators could choose to view NTT positions as 
a necessary temporary solution and thus not invest in supports or provide support in terms of pedagogical and procedural resources.

Sociopolitical culture is influenced by tacit assumptions and implied expectations contributing to the ambiguous nature of the workplace in postsecondary education. Schein (2016) outlines three levels to an organization's culture: artifacts, espoused beliefs and values, and underlying assumptions. These levels can be used to study the sociopolitical environment of the university and clarify the NTT experience. Artifacts include the visible tangible items that can be seen and heard including language (Schein, 2016). The language we choose to describe events or characterize positions sends implicit messages. Davis (2017) points out that at least two of the terms used to describe NTT are engrained with messages of separate and unstable by their very definitions. The word contingent is defined as "likely but not certain to happen" or "subject to chance" and the definition of adjunct is "something joined... but not essentially a part of it" (Davis, 2017, p. 6). The very title of the job is a reminder that NTT faculty are not something. Specifically, not tenure-track. The infrastructure of postsecondary education was designed for TT faculty. Positions that were viewed as temporary did not require permanent language or support systems. However, as evidenced by the rapid growth in the use of NTT faculty, these positions are no longer a temporary solution. Levin and Shaker (2011) agree that the terminology used to describe NTT faculty both full and part-time is considered exclusionary and derogatory, a reminder that NTTs are not truly a part of the professoriate. This was confirmed in their study of 18 full-time NTT faculty in English departments across multiple postsecondary institutions. They found that NTTs had dualistic orientations as prestigious experts in the classroom and feel diminished in their professional capacity among TT colleagues due to their lower status in the hierarchal system of the professoriate (Levin \& Shaker, 2011). The language used to describe 
NTT faculty is an artifact within postsecondary education that demonstrates the beliefs, environment, and value of and for the NTT faculty. Having access to resources such as computers and offices is another artifact that sends messages of value, importance, or unimportance to NTT employees. Organizations invest time and money in what they value. Much of this is intertwined with a university's beliefs. Schein's (2016) second level of understanding of an organization lies in espoused beliefs and values.

Organizational beliefs and values can be found in policies and procedures, both what is promoted and what is permitted. Schein (2016) explains these value systems as the foundation on which institutions build socially acceptable group norms. Beliefs and values are found in the constructs that are foundational to the core of the agency. Postsecondary education has norms built upon the expectations of teaching, research, and service. The primary value of importance to the professoriate is academic freedom. The American Association of University of Professors (AAUP) established the principles of academic freedom in 1940 with few changes since. At its base, academic freedom allows educators and students at publicly funded universities to teach and research without fear of repercussion (Poch, 1993). However, academic freedom is not a belief afforded to TT and NTT faculty alike (Kezar, 2013a). In part, due to how NTT faculty acquire their positions they may not be aware of the concept of academic freedom. Additionally, as Thompson (2003) points out positions that are reappointed from year to year often have few rights without collective bargaining and may face nonappointment without justification. The reliance on administration for appointment on a semester or annual basis shifts the relationship from one of freedom to one of necessity. Reichman and Scott (2019) cite cases of part-time NTT faculty having contracts canceled for voicing their concerns with the curriculum. It is because of academic freedom that faculty governance has importance, faculty are an integral part of the 
decision-making regarding the curriculum and decisions regarding academics. Full-time NTTs may be allowed to participate in faculty governance but not feel welcome, equally represented, or valued in doing so and part-time faculty may be excluded entirely (Gehrke \& Kezar, 2015; Morrison, 2008). NTT faculty are not afforded protections held as a fundamental right for TT faculty through academic freedom, however, they often have some opportunities to participate in faculty governance, but do not avail themselves of this. The question arises as to why? The answer may lay in the third level of culture Schein (2016) defines as tacit or underlying assumptions.

Tacit assumptions are implied or assumed norms based on core beliefs and values often evidenced through artifacts (Schein, 2016). These assumptions are difficult for outsiders to understand as there may be conflicting implicit and explicit messages. Things are not always what they seem on the surface. Further, social interactions have layers of meaning. Each social discourse is steeped in what Scott (1990) terms 'public transcripts', essentially the way dominant and subordinate parties interact in public spaces. These interactions are layered with 'hidden transcripts', the behind-the-scenes social nuances that represent the actual power struggle between groups (Scott, 1990). Regardless of appointment type, faculty navigate the workplace for the hidden patterns, appropriate social cues, and expectations of their roles. These assumptions pose a challenge for NTT faculty who are faculty but not fully a part of the organization's sociopolitical culture. Schein (2016) explains "The power of culture comes about through the fact that the assumptions are shared and, therefore, mutually reinforced" (p. 31). NTTs may be excluded from aspects of the professoriate due to scheduling, workload, job expectations, or explicit exclusion based on inaccurate assumptions. As a result, NTT faculty may not have opportunities to fully acclimate to the postsecondary environment. 


\section{NTT Pedagogical Preparation}

Despite being hired to teach, postsecondary institutions have no requirement that faculty be trained educators (Barney, 2019; Hansen, 2013). Designed to impart information related to discipline-specific knowledge and research skills, many scholars agree that doctoral education simply does not adequately prepare faculty to teach (Adams, 2002; Pace, 2017; Schmid, GillianDaniel, Kraemer, \& Kueppers, 2016). Faculty vary greatly in level of preparation from none to in-class observations, to graduate teaching assistant positions. The Center for Community College Student Engagement (2014) reports that in community colleges $11 \%$ of part-time and $18 \%$ of full-time NTT hold a doctoral degree. Unfortunately, comparable data for four-year institutions could not be replicated. The GAO report attempted to analyze educational attainment for NTT faculty, but the data was insufficient to include, thus the report states NTT have substantially fewer terminal degrees than TT faculty (Brown Barnes, 2017). The difference in degree requirements for hire would anticipate this result. Additionally, NTTs may teach with a Mater's level credential. Lacking specific data, it can only be stated that some NTTs hold doctoral degrees. Having a terminal degree, however, is an insufficient standard to measure pedagogical preparation for any faculty member. Postdoctoral programs that prepare faculty for careers in higher education have an emphasis on research and service but lack adequate preparation in pedagogical practices (Ahn, 2018; McKee \& Tew, 2013; Weimer, 2004). Most doctoral programs provide very little or no coursework and experience in pedagogy and teaching competency instead focusing on research (Cahn, 2008; Schmid et al., 2016). Graduate curriculums typically do not have room for pedagogical preparation in addition to rigorous courses on discipline-specific material and research methodology, however, in-depth research on doctoral program students teaching preparation is largely missing from the conversation (Barney, 
2019). The assumption is the training gained by pursuing a discipline-specific advanced degree is sufficient minimum qualification for teaching.

Lack of pedagogical training is not a new phenomenon. B.F. Skinner noted sarcastically in 1956 that "college teaching is the only profession for which there is no professional training" (p. 221). The fact that faculty are hired with content-specific degrees and no pedagogical training harkens to the early days of teacher education being viewed as low work. During this time, it was a commonly held belief that anyone can teach (Piccanio \& Spring, 2013). If the work of teaching, communicating, and conveying information is not a true profession the only essential training to teach would be discipline-specific content knowledge. Content knowledge is essential to the professoriate, but for those that strive to teach, pedagogy is crucial as well. That is not to say that those without educational degrees are incompetent teachers. Quite the contrary, succeeding in a profession despite formalized training demonstrates perseverance. While there are studies that evaluate student persistence when taking courses from TT versus NTT faculty, the literature is silent on the differences in pedagogical preparation specific to appointment type (Ran \& Xu, 2019). Studies demonstrate that faculty apply knowledge from self-observation, student feedback, and modeling skills observed from teachers they had when they were students to inform their pedagogical practices (Hativa, Barak \& Simhi, 2001, Weimer, 2004). Without formal training in pedagogy, faculty teach in ways that reflect their character, values, and preferences from past experiences with teaching (Weimer, 2004). Drawing on discipline-specific knowledge and blending it with pedagogy based on personal preference and experience may not prove effective. At best, this approach would be segmented and time-consuming as instructors use experience to find impactful methods. Oleson and Hora (2013) found that faculty use a variety of past experiences to inform their teaching, including modeling, and emphasize that 
instructional choices should not be simplified to a single process. Certainly, NTTs hired based on professional experience incorporate life experiences into their pedagogy as well as contentspecific knowledge.

NTTs vary greatly in their knowledge of pedagogy and are often hired due to either discipline-specific degrees and/or career experience in a specified field of study (Cahn, 2008). Teaching primarily introductory courses with large numbers of students full-time NTTs teach more courses than their TT counterparts (Baldwin \& Chronister, 2001; Merritt Boyd, 2016). With a position focused on teaching, pedagogy and preparation are foundational skills necessary for NTT faculty. Using the National Study of Postsecondary Faculty (NSOPF) to compare teaching strategies between full-time TT and full-time NTT faculty Baldwin and Wawrzynski (2011) explored how faculty used subject-centered and learning-centered teaching strategies, concluding that there are differences in teaching strategies across environments and between faculty appointment types. Full-time NTT faculty approach their teaching in similar ways to TT educators, however, part-time NTT educators did not use similar methods, this was attributed to the circumstances specific to appointment type (Baldwin \& Wawrzynski, 2011). This suggests that environmental factors such as resources available to NTTs may have an impact on pedagogy. Overall, there is insufficient data in the NSOPF, and the question as to how the NTT faculty perceive their preparation is currently unanswered. Umbach (2007) agreed that full-time NTTs and part-time NTTs differed in preparation with full-time NTTs spending more time preparing for class than TTs or part-time NTTs. Considering the primary duty of the full-time NTTs position is teaching and they may not be engaged in research or working outside of the university as their TT or part-time NTT colleagues are, this is not a surprising finding. 
The literature specific to NTTs is inconclusive on the effectiveness of NTTs as educators and often fails to distinguish between full and part-time appointments. Michel, Chadi, Jimenez, and Campbell, (2018) sought to determine if the classification of faculty appointment was the distinguishing factor in student achievement related to teaching and found that other factors such as class size, discipline, and departmental culture impact student experience more than tenure status. Essentially, context matters. The types of employment experiences faculty have regardless of tenure status impacts teaching. It is, therefore, adventitious for the administration to consider the orientation, evaluation, and professional development provided to faculty, specifically the new majority, NTT faculty. The NTT experience is a blend of pedagogical preparation, procedural knowledge, and sociopolitical factors that impact the day-to-day life of the NTT faculty member. How NTTs merge their past experiences, discipline-specific training, modeling, and knowledge acquired through orientation, evaluation, and opportunities for professional growth directly relates to their ability to acclimate to their roles in postsecondary education.

\section{Orientation, Evaluation, and NTT Professional Development}

Policies and procedures vary widely amongst institutions in the process of orientating, evaluating, and providing professional development to NTT faculty. The spectrum of experience varies from none to full inclusion in all aspects of faculty personnel operations. Most jobs include training on the duties and expectations to be performed. However, both part-time and full-time NTTs often do not receive formalized orientation and thus lack knowledge of resources to complete procedural tasks (Hart, 2011; Kezar \& Sam, 2010; Ott \& Cisneros, 2015).

Orientations serve to convey information to employees regarding institutional values, workplace expectations, and procedures for evaluation among other pertinent information to succeed in a new position. Historically, NTT faculty have been considered temporary and thus did not 
receive the same onboarding experience as their TT colleagues (Gappa \& Leslie, 1993; Umbach 2007). Researchers recommended support to NTT faculty include systematic performance reviews, professional development, and supports such as orientations (Baldwin \& Chronister, 2001; Gappa \& Leslie, 1993).

Performance reviews include both student evaluation and administrator appraisal of performance. Evaluations have an integral role in contract renewal for NTT faculty whose appointments are short in duration. Often NTTs are hired on a semester or annual basis with a potential for renewal. Thus, evaluation data becomes critical to NTTs that are unsure of reappointment. Additionally, faculty of all status types use results of evaluations to inform their teaching. The feedback provided allows faculty to determine areas for growth and future professional development. Evaluation data involves not only student feedback but may also include observation of teaching. Unfortunately, Kezar (2013b) conveys stories of administrators going through the motions by providing critiques to meet the required assessment for NTTs without substance, resulting in evaluations in name only. Baldwin and Chronister (2011) evaluated criteria for full-time NTTs evaluations including categories related to teaching expertise and affirm that evaluations are used specifically for relevancy in informing teaching practice for the NTT faculty member as well as a form of assessment for retention and rehire. Merrit Boyd's (2016) qualitative study of full-time NTT faculty showed that having unclear roles made it difficult for full-time NTTs to meet required contract renewal criteria. Essentially, being unsure of what the position expectations are makes it difficult to meet them and causes insecure work environments. To confirm this, Figlo, Schapiro, and Sorter (2015) found that NTTs that have higher job security perform better in areas of teaching. Despite their importance, few NTT faculty experience clear expectations, substantive feedback, or any evaluations (Bland et al, 
2006; Kezar, 2013b). The presence and quality of evaluations vary widely between campuses and even within the institution (Waltman, Bergon, Hollenshead, Miller, \& August, 2012). If NTTs are to remain in positions teaching in universities, then support should be provided for evaluation procedures as well as pedagogical support in the form of professional development.

Professional development includes not only orientations but also opportunities for growth in all aspects of one's role as a faculty member. Professional development and training may be available to NTT faculty but may not be offered at times that are conducive to the NTT that is a Specialist holding another full-time job outside the university or the NTT Freelancer working for multiple institutions (Baldwin \& Chronister, 2001, Keezar, 2012). A limited number of studies explore professional development specifically for NTTs. Noting the complex nature of NTT faculty positions and the variant definitions of professional development, de Saxe Zerden et al, recommend faculty learning communities as a cohort method of professional development (2015). While learning communities have limitations of time commitments, support from peers could speak to connecting NTT faculty to their colleagues and the institution. The institution may not invest in part-time NTTs as they may not view them as a permeant part of the university, however, researchers have suggested that development opportunities for both full and part-time NTTs cannot only aid in developing skills, but also contribute to a sense of belonging, professionalism, and value (Eagan, 2015; Kezar, 2013; Levin \& Shaker, 2011). Determining professional development needs for NTTs may prove as diverse as the individuals themselves, however, not trying may prove detrimental to the university as a whole. Orientations, teaching evaluations, and ongoing opportunities for professional development have all been suggested as best practices in acclimating NTT faculty to their positions in postsecondary education (Kezar, 2012). 


\section{Conclusion}

To understand the complexities of the NTT faculty experience it is necessary to first explain the current university structure that relies heavily on the NTT appointment. This literature review first explored the history of the professoriate. This historical perspective aids in providing a rich description of the context in which the phenomena will be explored. This information is important in qualitative studies aimed at exploring the inner experiences and meanings of the participants (Corbin \& Strauss, 2015). Qualitative researchers rely on the participants' point of view historically and socially to inductively create meaning from phenomena observed (Creswell \& Creswell, 2018). The literature used in Grounded Theory best informs and contextualizes the study (Goulding, 2008). Much of the literature regarding NTT faculty centers around the causality and the numbers of NTT appointments and is quantitative (Bergman, 2011; Holler, 2014; Kezar, 2014; Hensely, 2016). The literature pertinent to the experience of being an NTT faculty member is limited and can be categorized by knowledge and functions of both procedural and pedagogical nature within sociopolitical constructs. While job duties such as teaching and attending faculty meetings can be clearly delineated, the sociopolitical factors impacting NTTs are more nuanced. This study will strive to gain an understanding of the context-bound and value laden experiences of NTT faculty work (Goulding, 2008). The work of Gappa and Leslie (1993) helps to provide insight into the variety of full and part-time NTTs. Considering the rapid growth in the utilization of NTT positions, universities were underprepared to provide adequate supports in orientation, evaluation, and professional development. Current research has focused on the number of NTT positions, job demands, and pay equity (Feldman \& Turnley, 2001; Fuller, Brown, \& Smith, 2017). Limited literature is available in the realm of understanding the perspectives of the NTTs, their experience, and 
expectations of NTT positions in the context of their environment. This study will fill a gap in the exigent literature by providing a perspective from the NTT faculty themselves regarding their preparation, pedagogical support, and evaluation. This information could be used to bolster the university support systems for an ever-growing segment of the faculty ranks. 


\section{CHAPTER III: METHODOLOGY}

The previous two chapters provided the background for the study and outlined how the research fits within the wider literature. This chapter outlines the methods used to accomplish the task of representing the NTT faculty experience specific to their perceptions on pedagogical preparation and support inclusive of professional development and evaluations. The research questions were evaluated through an interpretivist paradigm using Grounded Theory methodology. The methodology section outlines the participant selection, data collection, instrumentation, data analysis, and addresses limitations in trustworthiness and bias.

\section{Research Questions}

The research questions that guided the study are outlined below.

RQ 1: How pedagogically prepared did NTT faculty feel when they began teaching at the postsecondary level?

1a. What types of previous experiences or pedagogical training inform NTT perceptions of preparedness?

RQ 2. How do NTT faculty describe the pedagogical support they receive in their teaching roles?

2a. What is the role of professional development in supporting NTT faculty teaching?

2b. How are evaluations used to inform NTT faculty teaching and retention?

2c. What additional supports do NTT faculty desire to improve their teaching?

To date, little is known about the pedagogical preparation of NTTs and their perceptions regarding this aspect of their positions. Much of the current literature regarding NTT faculty 
perceptions focuses on how NTTs contend with poor working conditions, lack of support, and/ or economic inequity (Banasik \& Dean, 2016, Baldwin \& Wawrzynski, 2011). A gap in the current research on NTTs exists relevant to NTT faculty perceptions of their experiences specific to pedagogical preparation. Umbach (2007) examined the effectiveness of NTTs teaching methods in working with undergraduate students but did not inquire to the NTT faculty themselves regarding their perceptions on preparedness. This study served to fill this gap with the end goal of providing insight into NTT faculty experiences and needs regarding teaching. Previous experience, pedagogical training, and pedagogical support are combined as foundational building blocks NTT faculty use to inform their teaching. The interpretivist paradigm frames this study with a constructivist epistemology. This chapter will outline the interpretivist paradigm, the use of Grounded Theory, and the research design of the study. Finally, the chapter will discuss ethics and trustworthiness.

\section{Interpretivist Paradigm}

The underlying assumption of this study was that NTT faculty construct new knowledge based upon previous knowledge. Interpretation is the means of this construction (Hussein, Hirst, Salyers, \& Osuji, 2014)). Researchers rely on the participants' point of view, historically and socially, to inductively create meaning from phenomena observed (Creswell \& Creswell, 2018). In this study, NTT faculty perceptions formed from their previous experiences and/or pedagogical training can help to illuminate how NTTs perceived their preparedness to teach in postsecondary education. Knowledge generated from this study can help make sense of how past experiences and professional development intersect with current teaching. In turn, NTT faculty are evaluated and retained based on their teaching. Thus, the concepts are interrelated. 
The interpretivist paradigm aided in understanding the context in which the NTT faculty experience teaching and preparation. This paradigm postulates that multiple value-laden realities exist within sociopolitical constructs and is closely associated with Grounded Theory (Goulding, 1998). Interpretivism evaluates ways of knowing including language, gestures, actions, and expressions, and is useful in exploring the artifacts, espoused beliefs and values, and underlying assumptions that NTTs use to contextualize their preparedness and pedagogical supports (Goulding, 1998; Schein, 2016). The working construct in this study was that faculty incorporate knowledge gained from past experiences into future iterations of similar activities. People seek meaning in their experiences and learn from one experience to the next. As this study relied on the participants' responses to create interpretations, Grounded Theory guided the inquiry.

\section{Grounded Theory}

Grounded Theory is a qualitative methodology of inquiry that allows the researcher to enter a situation without predisposed theories, thus allowing the researcher to develop explanations based on the data gathered (Corbin \& Strauss, 2015). Using this methodology, the researcher attempts to understand the world of the participant by studying their beliefs and experiences. The theories developed are 'grounded' in the experiences of the research participants based on multiple data points. Seeing as the aim of this study was to explore perceptions of NTT faculty regarding preparedness and considering the lack of current research on perceptions for this group, Grounded Theory was most suited. Grounded Theory can help researchers explore phenomena not yet discovered including how participants experience and discover meaning (Corbin \& Strauss, 2015) It is of note that in true Grounded Theory one is creating new theoretical foundations where little is already known (Goulding, 2008). A new theory may or may not emerge as a result of a Grounded Theory study; however, this would lend 
the study to using modified Grounded Theory. The modification of Grounded Theory is one in which the researcher utilizes Grounded Theory in both process and methods, but the research does not necessarily culminate in a new theory. In these cases, a collection of key concepts will be presented that emerged from the data. The result of this study suggests a shift in the current model of the professoriate where NTTs are supported as professionals for the merits of their positions and not compared to their TT colleagues.

Hallberg (2006) suggests that Grounded Theory be used fluidly. One example of such fluidity is the process of constant comparison. The researcher evaluates data gathered continually throughout the study. This formative approach can be utilized to make modifications in the study during the process. This allows for discovery during the research study through the unfolding of events. For example, this study proposed to discover NTTs' perceptions regarding their preparedness to teach and pedagogical supports. It became apparent during interviews that the supports requested by NTTs expanded beyond pedagogy. Thus, the researcher began asking about supports in general as well as those related to pedagogy. Embracing Grounded Theory allowed for an in-depth view into the NTT experience overall and not just limited to pedagogy. This methodology produced rich insights into the world of the NTTs.

Grounded Theory is best used when little is known about a phenomenon (Bowen, 2008). Currently, little is known about the perceptions and experiences of the NTT faculty member regarding pedagogical preparation and desired pedagogical supports. Grounded Theory offered an opportunity to gather thick descriptions and detailed data. The depth of this data was then systematically coded for understanding. Hussein, Hirst, Salyers, and Osuji (2014) note advantages of using Grounded Theory such as creativity, rich and deep data, and systematic collection and analysis of data that brings rigor to the process. The exploration of perceptions 
aligns with Grounded Theory and thus it was utilized to guide the research process and its design. The research design including data collection, instruments, participant selection, and method of data analysis follows.

\section{Research Design}

\section{Participants}

Studies of NTTs are better able to make recommendations if they focus on a particular institutional type, are mindful of disciplinary differences, and distinguish demographic differences that may impact the NTT experience (full versus part-time appointments, for example) (Kezar, 2013b). In terms of institutions, four-year teaching-focused universities with tighter budgets use NTTs more often than privately funded or R1 institutions (Kezar, 2013b). For this reason, this study was limited to a state-funded Midwestern university with a teaching focus. According to Planning, Research, and Policy Analysis (2019), the number of TT faculty was 744 and the number of NTTs was logged at 628 inclusive of both full and part-time. It is unclear if this number includes graduate assistants. Additionally, participants were identified by department, comparing NTTs against one another in the same discipline. Demographic differences in NTTs were classified using the Gappa and Leslie (1993) categories of Career Ender, Aspiring Academic, Freelancer, and Specialist rather than focusing solely on part and full-time status.

Three unique departments in three different academic colleges were selected for this study. Departments were selected based on the number of NTTs used making the probability of participation greater. For example, department C currently uses 48\% NTT faculty (Planning, Research, and Policy Analysis). This represents the largest number of NTTs on campus. This 
department has gradually utilized NTTs to teach the majority of its lower division and general education courses. Additionally, department $\mathrm{C}$ offers a large number of courses that are general education, and thus most undergraduates at the institution would have contact with this department. Department $\mathrm{C}$ is in the College of Arts and Sciences. To be attentive in selecting participants from divergent disciplines, additional academic programs were selected for participation one in the College of Applied Sciences (department A) and one from the College of Education (department B). Department A is one of the largest on campus and hires both part and full-time NTTs. Department B is one of the signature programs of the university and thus has an important presence on campus. These three colleges represent a variety of academic foci. A sample of three departments provided a large enough group of participants for saturation of the data to occur. Creswell and Creswell (2018) explain that in qualitative research the number of participants is not as relevant as the quality of the information gathered from the participants. Thus, this study interviewed participants until clear themes and commonalities were found that begin to repeat known as saturation. A total of 15 NTTs, five from each department were interviewed.

Gappa and Leslie (1993) found that part-time/full-time status may have an impact on the teaching experience and thus both appointment types were included in the participant pool. Graduate students were excluded from the study participants. The researcher requested administrators in each department to provide departmental rosters of NTTs that were teaching at least one course at the time of interviews. The researcher then emailed potential NTT faculty participants from departmental rosters and requested interviews. As NTT faculty tend to be a tight-knit group, the researcher planned to request that interviewees suggest additional names of colleagues that would be willing to participate in the study. Gaining participants by 
recommendation from current participants is known as a snowball sampling technique. The participants would collectively grow in number based on the previous participant recommendation. Subsequent participants are then asked to identify others like themselves that may be suitable for the study (Merriam \& Tisdell, 2016). Snowball sampling would increase the potential list of participants but as the response rate was high this method proved unnecessary. The first five NTT respondents from each department were scheduled for interviews. Additional names were maintained in the event that further data were needed. This was not used, as the researcher believed saturation was achieved through the five participants in each department.

Additionally, study participants included university employees that hire, evaluate, or work closely with NTTs, labor union representatives, and faculty developers. These groups were selected to provide secondary data and serve as points of triangulation. While NTTs provided primary data on their perspectives, administrators, labor union representatives, and faculty developers supplied information that was used to fully understand the sociopolitical environment in which the NTTs work. Additionally, administrators assisted in insights regarding policies and procedures including information on evaluations and retention procedures.

University employees that are charged with the task of selection, evaluation, or working closely with NTT faculty were interviewed from each department. These individuals were selected based on the tasks performed within the department, regarding management functions with NTTs. As departmental policies may vary on how NTT faculty are selected and retained, the researcher contacted each department individually and inquired as to the person or persons tasked with the above-mentioned responsibilities. Additionally, personal contacts and recommendations from colleagues were used to ensure the responsible and appropriate individuals were selected. One administrator was interviewed from each department $(\mathrm{N}=3)$ 
providing insights into NTTs evaluations, hiring, retention procedures as well as requests for supports received from NTTs both pedagogical and procedural. These participants contributed to the NTT story of procedural and sociopolitical factors influencing how NTTs described the support they receive in their teaching roles.

Another group of individuals who contributed to the details of the NTTs story was faculty developers. Faculty developers provide resources to meet the continuing educational needs of the faculty as a whole, including those faculty in NTT positions. Faculty developers within the faculty development center and those employed within the Department of Human Resources were contacted to explore pedagogical supports offered to NTT faculty. Faculty developers provided information to contribute to a deeper understanding of the NTTs' experience of preparedness to teach and the role of professional development in supporting NTTs' teaching. The faculty development center administrator was contacted to determine the most appropriate person to provide information on campus-wide development offerings for NTTs. The same selection method was followed for the department of Human Resources. Using this selection method, four faculty developers were contacted for interviews, two from Human Resources and two from the Faculty Development Center.

Lastly, the NTTs in this study are represented by a labor union. The researcher contacted all the labor union board members via email and inquired as to the most appropriate person(s) to interview. A union representative would add depth and detail to understanding the sociopolitical environment impacting NTTs employed at this institution. The emails went unanswered. Telephone requests occurred as a follow-up. One union representative responded for an interview. All other union representatives contacted either did not respond or declined to be in the study. The union representative interviewed did provide information regarding formalized 
procedures on evaluation, retention, and pedagogical support as well as thick descriptions of the sociopolitical environment. However, with only one respondent there was insufficient data. Further, to protect this individual's anonymity, they are not directly quoted in the findings. However, their perspectives are included through memos. Memos from the interview with this individual were used along with artifacts publicly available from the labor union to ensure this perspective was included.

A total of twenty-three participants were interviewed for this study including administrators and NTTs from three distinct disciplines as well as faculty developers and labor union representative. The three departments used in this study have several commonalities and differences. First, each department was unique in its discipline and thus would have contentspecific degrees required by its faculty. Second, each department fell into a different college, or organizational unit of similar departments clumped together for reporting structure. Departments represent unique disciplines within education, arts and sciences, and applied sciences and technology The similarities were in the fact that each department employed both TT and NTT faculty. The NTT faculty are both full and part-time. Departments were given a random alphabetical identifier and will be referenced throughout the study as Departments A, B, and C. Table 1

Department Administrators
Department
Administrator

$\begin{array}{ll}\text { A } & \text { Pat } \\ \text { B } & \text { Alex } \\ \text { C } & \text { Casey }\end{array}$


Table 1 shows the pseudonyms assigned for the three department administrators interviewed.

Due to the small sample size, gender-neutral names and pronouns will be used to further protect anonymity.

NTT faculty in the study included both full and part-time employees from three distinct disciplines. Graduate students were excluded from the sample. An equal number of NTTs was represented from each department, for a total of 15 NTTs.

Table 2

NTT Participants Descriptive Characteristics by Department, Full/Part-Time, Status and Gappa and Leslie Categories

\begin{tabular}{|c|c|c|c|c|}
\hline Department & Pseudonym & Full/Part-time & Status & Categories \\
\hline A & Isabella & Full & $\mathrm{N}$ & Specialist \\
\hline $\mathrm{A}$ & Sandra & Full & $\mathrm{Y}$ & Specialist \\
\hline A & Jada & Full & $\mathrm{Y}$ & Specialist \\
\hline A & Gretchen & Full & $\mathrm{Y}$ & Aspiring Academic \\
\hline A & Sara & Full & $\mathrm{Y}$ & Specialist \\
\hline B & Marcus & Full & $\mathrm{N}$ & Career Ender \\
\hline B & Vanessa & Part & $\mathrm{N}$ & Aspiring Academic \\
\hline B & Jake & Part & $\mathrm{N}$ & Specialist \\
\hline B & Maya & Part & $\mathrm{N}$ & Freelancer \\
\hline B & Maria & Full & $\mathrm{N}$ & Freelancer \\
\hline $\mathrm{C}$ & Latoya & Full & $\mathrm{Y}$ & Specialist \\
\hline $\mathrm{C}$ & Dan & Full & $\mathrm{N}$ & Specialist \\
\hline
\end{tabular}

(Table Continues) 
(Table Continued)

$\begin{array}{ccccc}\text { C } & \text { Gina } & \text { Full } & \text { Y } & \text { Aspiring Academic } \\ \text { C } & \text { Jason } & \text { Full } & \text { Y } & \text { Freelancer } \\ \text { C } & \text { Juanita } & \text { Full } & \text { Y } & \text { Freelancer }\end{array}$

Table 2 provides pseudonyms for participants in the study along with their full or part-time status, contractual status, and self-identified Gappa and Leslie (1993) categories. Participants were given the option to select a pseudonym. Most chose to use a pseudonym assigned by the researcher. Five NTTs were interviewed from each department. NTTs in the study included 12 (80\%) full-time and three (20\%) part-time. As there are large variances in how part versus fulltime is determined both in the literature and by participants, NTTs that held a 1.0 full-time equivalent (FTE) position at the time of interview as was self-identified were classified as fulltime. FTE varies from semester to semester for NTTs that have not reached the negotiated title of status (described next). The number of courses assigned to an NTT faculty member is based on need as is identified by administrators' prediction of course enrollments and the number of TT faculty available to teach in each semester. Any participant without status could change from full to part-time or not be employed by the university in subsequent semesters. Full and part-time status is fluid for NTT faculty therefore the title of status was most relevant in considering job security for NTTs and will be described next.

Eight $(53 \%)$ of the NTTs had received the title of status. Status is a longevity benefit at this institution that is negotiated by the NTT labor union. According to the labor union contract (Agreement, 2017), once the NTT employee has worked eight consecutive semesters the employee receives a pay increase and has additional job security. This security comes in the form 
of prioritization for class assignments before their non-status peers. Essentially, there is an order to be followed in course assignments. If the department administrator does not have a TT faculty to teach a course, they would first hire a qualified status NTT employee in their department, and then last a non-status NTT employee would be offered the class. Thus, obtaining status is the equivalent of the completion of an eight-semester probationary period that results in consistent course assignments. Seven (46\%) of the participants did not have status. Not having status means the NTT faculty member is not guaranteed work from semester to semester.

Lastly, the categories defined by Gappa and Leslie (1993) outlined in chapter one guided participant characteristics. These categories were used due to the large variance in how the terms part and full-time are defined in the literature and by individual NTTs. The Gappa and Leslie (1993) categories served as an important classification system in identifying the NTTs into groups with similar perspectives.

The researcher read definitions of the Gappa and Leslie (1993) categories and participants were asked to select one primary categorization that they felt best represented them based on their identity at the time of the interview. If the participant felt they could fall into one or more categories, they were prompted to select a primary category. Again, this was based on perception at the time of the interview only as NTTs may change from one category to another as perspective and motivations for employment change.

Representing the smallest number in the study was one (6\%) Career Ender. Career Enders were defined as NTT faculty who are retired and teaching courses to fill in temporarily. These NTTs do not intend on working full-time. Career Ender, Marcus, retired from a full-time position off-campus as a practitioner in his discipline and fit the definition well. 
Aspiring Academics are defined as NTT faculty that are in search of full-time academic appointments, may hold terminal degrees, and may teach courses at multiple institutions to piece together a wage-earning job. Three (20\%) individuals in the study held terminal degrees or were working towards completion of terminal degrees consistent with the definition of Aspiring Academics. While other NTTs in the study had terminal degrees, these three stated that they were actively pursuing TT positions and thus the description of Aspiring Academic appealed to them. All the participants that identified with this classification assumed this meant TT faculty appointment and did not focus on the part of the definition working at multiple institutions.

A total of four participants (26\%) were classified as Freelancers defined as NTT faculty that choose to teach part-time for the benefit of having a flexible schedule. Freelancers might teach at multiple institutions. While three individuals selected the category Freelancer due to working at multiple institutions, one participant did not feel he fit any of the categories cleanly. However, when describing his work, he explained that he chose to work part-time at multiple institutions. As this description is most consistent with the title Freelancer the researcher placed him in that category. The Freelancers either taught at multiple institutions or worked full-time in a nonacademic position off-campus in addition to their full or part-time NTT faculty role on campus.

Seven (47\%) participants identified as Specialists defined as NTTs with disciplinespecific expert knowledge. These individuals by Gappa and Leslie definition may teach full-time or have a career in a related profession and teach contingently. The participants in the study that identified as Specialists focused on the expert knowledge portion of the definition. There were participants in each of the three departments in the study that held degrees specifically in pedagogy, education, or teaching and learning. Participants with pedagogical degrees have 
specialized knowledge, at least as teaching professionals. However, the pedagogical degree was not used for classification, rather participants self-selected a descriptor from the defined list of categories. It was originally believed that having a pedagogical degree would be significant in the study, however, the security of status proved more relevant than having a teaching degree. Additionally, there were participants with teaching degrees that did not identify as Specialists. The defining factor for Specialists was possessing the content specific expert knowledge in their specific discipline relevant to the courses they have taught at the university.

Numerically, this study represents a sample of the NTT faculty on campus with Specialists being the largest group and Career Enders being the smallest. Career Enders and Aspiring Academics have definitions connected to life milestones of retirement and dissertations and thus make these two categories the most easily identifiable for participants to classify and identify. Specialists identified most with discipline-specific expert knowledge component of the definition. Freelancers focused on the multiple institutions component of the definition rather than the part-time or motivation of flexible schedule portions of the definition. All three administrators concurred that there were individuals in each of the Gappa and Leslie (1993) categories reflected in their departments NTT faculty. Administrators stated that Career Enders would be the least number represented and Specialists the highest. Faculty developers stated that they provided services for NTTs matching all definitions and they agreed that the Specialists would be the most prevalent and Career Enders the least. The labor union information also reflected that NTTs on this campus fit into all categories but the union representative interviewed identified Specialists as the smallest number.

Alumni status emerged as an important defining characteristic in the NTT experience as will be discussed in the findings. 
Table 3

NTT Participants Alumni Status

\begin{tabular}{|c|c|c|}
\hline Department & Pseudonym & Alumni \\
\hline A & Sandra & $\mathrm{N}$ \\
\hline A & Jada & $\mathrm{Y}$ \\
\hline A & Gretchen & $\mathrm{N}$ \\
\hline $\mathrm{A}$ & Isabella & $\mathrm{Y}$ \\
\hline A & Sara & $\mathrm{Y}$ \\
\hline B & Marcus & $\mathrm{Y}$ \\
\hline B & Vanessa & $\mathrm{N}$ \\
\hline B & Jake & $\mathrm{N}$ \\
\hline B & Maya & $\mathrm{Y}$ \\
\hline B & Maria & $\mathrm{N}$ \\
\hline $\mathrm{C}$ & Latoya & $\mathrm{Y}$ \\
\hline $\mathrm{C}$ & Dan & $\mathrm{Y}$ \\
\hline $\mathrm{C}$ & Gina & $\mathrm{Y}$ \\
\hline $\mathrm{C}$ & Jason & $\mathrm{Y}$ \\
\hline $\mathrm{C}$ & Juanita & $\mathrm{Y}$ \\
\hline
\end{tabular}

Table 3 reflects NTT participants' alumni status organized by department. Ten (66\%) of the 15 NTT participants were alumni of the institution in which the research occurred. This included either undergraduate or graduate work at the masters or doctoral level. Currently enrolled students that had yet to complete a degree with the institution were not categorized as alumni. 
While alumni status was not predicted as a relevant characteristic it emerged as a relevant characteristic in how NTTs become accustomed to their workplace.

Table 4

NTT Participants Gender

Department Pseudonym Gender

A Sandra $\quad$ F

A Jada $\quad$ F

A Gretchen $\quad F$

A Isabella $\quad \mathrm{F}$

A Sara $\quad$ F

B Marcus M

B Vanessa $\quad$ F

B Jake M

B Maya $\quad$ F

B Maria $\quad F$

C Latoya $\quad$ F

C Dan M

C Gina $\quad$ F

C Jason M

C Juanita $\quad$ F

Table 4 reflects NTT participants' gender organized by department. Eleven (73\%) of the participants self-identified as female and four (26\%) as male. While gender was not specific to the study's research questions. It was a characteristic that was noted to consider during the 
analysis stage and potential differences in responses. However, based on participant responses, gender did not appear to play a prominent role in impacting the NTT experience.

The final group of participants interviewed was faculty developers. These individuals organize courses and workshops to meet the continuing professional development needs of the faculty including both TT and NTT appointments.

Table 5

Faculty Developers

Department $\quad$ Faculty Developer

$\begin{array}{cc}\text { Human Resources } & \text { Karen } \\ \text { Human Resources } & \text { Tyler } \\ \text { Faculty Development Center } & \text { Janelle } \\ \text { Faculty Development Center } & \text { Sue }\end{array}$

Four faculty developers participated in interviews as is reflected in table 5. Two participants provided professional development services on behalf of the Human Resources Department. Human Resources focuses on a benefits orientation for all TT and eligible NTT faculty.

Eligibility is defined as those that meet the state's eligibility requirements for insurance purposes. Additionally, an optional acculturation orientation is offered to all faculty. This Human Resources program provides background and culture on the university. Two participants worked in the Faculty Development Center offering professional development in the form of continuing education. The Faculty Development Center coordinates a fall orientation for all faculty as well as ongoing development. The ongoing development focuses on technology such as how to use 
the universities course management system as well as pedagogical assistance and classroom management.

Lastly, the entire labor union board were contacted for interviews. One board member agreed to participate in the study. The researcher chose not to give this participant a name or include their direct information in the study to protect their anonymity. Instead, information from this participant is presented in aggregate with union artifacts and memos. A total of twenty-three individuals participated in this study. As the research questions focused on NTTs' perceptions as well as support, selecting participants that are NTTs as well as those in critical roles to provide or decline support aided in a thorough analysis of the NTT experience.

\section{Data Sources}

Data includes the pieces of information that are collected, observed, and analyzed in an environment that are of interest to provide insight into a phenomenon or experience. Merriam and Tisdell (2016) characterize data as ordinary bits until someone takes interest and finds patterns. For this study, semi-structured interviews, and document reviews, along with descriptive information were used to collect the bits of information that gave insight into the research questions.

Data for this study included twenty-three interviews, artifacts, and memos. The NTT faculty interviews served as primary data sources to gather NTTs' perspectives. Interviews with faculty developers, administrators, union representatives as well as artifacts serve as secondary data to assist with triangulation. NTT interviews aided in answering research questions specific to NTT perceptions (R1 \& R2 and all sub-questions). Administrator interviews were helpful in determining the types of supports offered to NTTs, how evaluations are used to determine retention and supports most often requested by NTTs (R2 and sub-questions). Regarding R1a, 
the types of training that inform NTTs' perceptions, administrators contributed by discussing the level of preparation they prefer in new hires. Faculty developers were most insightful in R2a the role of professional development in supporting NTTs. The interviews with faculty developers were compared with those of administrators and NTTs to make sense of how professional development is used and perceived by NTTs. The union representative interview and artifacts including the labor union agreement and department-level appointment and evaluation documents were used to inform research question $2 \mathrm{~b}$, how evaluations are used to inform teaching and retention.

\section{Semi-Structured Interviews}

The researcher conducted twenty-three semi-structured interviews during the course of the study. Interviews occurred with Administrators, faculty developers, NTTs, and a union representative as were described in the participant section. Interviews took place face to face, telephone, and zoom, selected by the participants. The interviews began with descriptive openended questions and proceed to semi-structured questions based on scripts approved by the Institutional Research Board (IRB) (Appendix A, B, \& C). While it was not possible to predict all the questions that were asked during the interview, some structure was provided based on the theoretical framework and research focus of the study. Descriptive questions included items such as when the person began working at the institution and classification for NTTs based on the Gappa and Leslie (1993) categories. Administrators, faculty developers, and a union representative were asked similar questions with the variation 'how would you classify the NTTs that work in your area'.

Semi-structured interviews allow individual participants to share their interpretations and perceptions of their experiences (Merriam \& Tisdell, 2016). Semi-structured interviews provide 
flexibility in using a predetermined list of topics but allow the interviewer to probe with followup questions (Corbin \& Strauss,2015). This method also allows participants to discuss items they feel are relevant but focused on a prescribed topic. This interview method is consistent with the goals of the study. While this method did generate unneeded information, it also allowed participants the flexibility to follow up with any information they thought relevant to the phenomena. Having a list of topics served to focus the interview (Appendix A, B, \& C). For example, all participants were asked about evaluations. Some chose to explain the procedure for NTT faculty evaluations only and others expanded upon their answers to describe how evaluations are used, the merits of evaluation, their preferences and emotions about evaluations, and the like. Using follow-up probes the researcher was able to note deeper explanations of phenomena.

To develop rapport, the interview began with predetermined descriptive categories. Starting with general information helps participants to relax and open up before more complex questions are asked. As a current NTT faculty member, the researcher connected with other NTTs based on the number of years taught and common experiences. This comradery proved assistive in helping participants share their stories and experiences. Predetermined descriptive categories from Gappa and Leslie (1993) also aided in structure and added context to the participants' experiences and data analysis. For example, full-time NTTs encountered different orientation and departmental preparation than part-time NTT faculty. Descriptive information was asked regarding the number of courses, the number of postsecondary institutions, and the disciplines in which the participant currently teaches. Participant classification into Gappa and Leslie's (1993) categories not only aided in describing the sample but also were used to further 
interrogate the data based on the current prevailing categories of Career Ender, Specialist, Freelancer, and Aspiring Academic.

Topics for the semi-structured interview centered around understanding the experience of teaching on the non-tenure track. Interview questions were generated based on the research questions and were designed to aid in triangulation of the data. The NTT faculty questions asked about perception of preparedness at hire, support in teaching, professional development, and evaluations. Interview questions for administrators mirrored those of the NTT faculty for consistency but gathered information from the lens of an individual charged with hiring, training, and evaluating NTTs. To add a third perspective to understanding the NTT faculty experience, faculty developers were asked similar descriptive questions, but the topics of these interviews focused on orientation, pedagogical support, and professional development. Finally, as the NTTs on this campus are represented by a labor union, a representative was interviewed to determine additional insights relevant to the study. This participant was asked very similar questions (Appendix D) but provided perspective through the lens of the union.

Follow-up probes for all four groups were related to procedural, pedagogical, and sociopolitical experiences that impact the NTTs' ability to integrate into the postsecondary environment. These probes allowed Grounded Theory to be used to discover new insights. For example, the question "What support did you receive to help you in your teaching role at the university?" was often answered with procedural or sociopolitical insight rather than specific to pedagogy. Interview questions were then altered slightly to ensure participants could answer not only with pedagogical support but also with any supports they felt they had perceived as relevant. This approach produced a deeper understanding of the experiences of the participants. 
All interviews were audio-recorded and transcribed for coding. Summaries were emailed to participants for member checking to ensure the accuracy of content and intent.

\section{Artifacts}

In addition to interviews, data was collected via a document review of artifacts. Artifacts can serve as reliable sources of information, especially when inquiring into formalized policies and procedures (Merriam \& Tisdell, 2016). The researcher inquired during the interviews with administrators as to any procedural, pedagogical, or sociopolitical artifacts that could assist in understanding the NTT experience within the scope of the research questions. These documents included items relevant to evaluation, retention, and pedagogical support including the labor union contract and individual departmental documentation on appointment, salary, promotion, and tenure policies. Following the guidelines from Merriam and Tisdell (2016) artifacts were reviewed for authenticity, completeness, and relevance to the study. Artifacts analyzed included the labor union agreement, departmental evaluation documents and appointment, salary, promotion, and tenure policy documents. The labor union contract was available on the universities' Human Resources website and provided insights into evaluation processes and purposes as well as orientation, promotion, and retention procedures. For example, a section of the labor union agreement regarding orientations was informative in learning that the university and labor union agree that a departmental orientation is to occur as well as a general university orientation. The excerpt from the agreement follows.

Section 10.05: Departmental Orientation for New NTT Faculty Members The Department will provide new NTT faculty members with information about departmental operations, procedures, and expectations within the new NTT faculty 
member's first thirty (30) days of employment. This information will be in addition to the general University orientation provided by Human Resources.

This portion of the contract shed light on stated expectations and allowed the researcher to inquire of administrators and NTTs to their lived experiences for comparison. This data worked together to further add to triangulation.

Each administrator interviewed was asked to provide departmental artifacts related to NTT hiring, evaluation, and reappointment. All three departments had appointment, salary, promotion, and tenure (ASPT) policy documents for their TT faculty. Department A provided one such document for the NTT faculty. This document aided in understanding the power hierarchy in Department A as well as information on evaluation and retention. Administrators in Departments B and C provided samples of departmental form letters, emails, and evaluator forms that helped to communicate the evaluation process for NTT faculty. Lastly, information that contributed to understanding current onboarding and orientations for NTTs included an outline from the administrator in Department B and one from a faculty developer in Human Resources. Artifacts from each of the three departments and faculty developers served as additional points of data and contributed to triangulation in the study.

\section{Memos}

Lastly, memos were used as a point of data. The researcher wrote a memo on each interview as well as used memoing to reflect on key insights as the study progressed. Additionally, memos were written reflecting on key artifacts such as the labor union agreement. Memos were used to reflect, organize ideas, and question concepts. Memoing is a way to recall details that may otherwise be forgotten about past events. It serves for reflection and aids in triangulation through ensuring coherent themes (Creswell \& Creswell,2018). Memos in this 
study ranged in length from a few sentences to multiple pages. Considering the interviews began over a year from the writing of this chapter, they have proven invaluable sources of data. To demonstrate the contents of memos two examples, follow. The first reflects how reflection can occur. This memo was written as the interviews were concluding.

August 1, 2020

There seem to be so many stories here. What is the one that needs to be told?

What do you need to feel prepared? Valued? Vested? The power struggles and insecurity play into the reality of needs. If you don't feel safe and secure, where do you turn?

You love this job and want it and want more so you will tolerate being heaped on or not getting enough when there isn't formal support the NTT turn to each other. Why? what's going on?

This memo shows the researcher grappling with the data and questioning motivations and what the meanings are to the information gathered. The second memo is longer and demonstrates how memos were used to aid in clarifying themes that emerged from the data. This memo was written after interviews while coding data.

March 20, 2021

Lacking a formalized orientation and mentorship program both PT and FT NTT form tight informal networks to support one another. This appears to have grown out of necessity. While this support fills the need for some, others may still be left out. Especially in departments that have hierarchies beyond the TT/NTT division. Some areas have reported further demarcation of NTT who are PT vs NTT that are FT and even NTT that teach general education courses versus courses in the major. 
The NTT compares personal experiences in an effort to navigate the unspoken expectations of employment at the university. While universities do not treat FT and PT NTT the same there are also great variances from department to department and in the types of unspoken expectations. NTTs also notice inconsistencies both within and across departments and this adds to the feeling of insecurity. For example, a course with multiple sections is often perceived as inconsistent in curricular delivery. Another example is some departments omitting NTTs from faculty meeting invites and others have an expectation that they should attend. The informal network shares this information and is unsure what it means. Do I have to attend if my friend in another program doesn't go to their meetings? Further, if I do these things do I increase or decrease my chances of being hired back next semester.

It is unclear if these are cultural creations of individual disciplines or tacit assumptions. However, when lacking explicit information from supervisors the NTTs attempt to understand their role on campus through observation and conversation with one another. Is this typical of how people acclimate to new environments? Or unique for NTTs?

This second memo demonstrates the process of narrowing themes. Corbin and Strauss (2015) suggest memos can be used to outline the findings of a study. Memos were used in this study to clarify concepts and organize findings.

\section{Data Analysis}

The data were analyzed both inductively and deductively. Corbin and Strauss (2015) explain that no researcher enters a situation with a true blank slate even using Grounded Theory. Thus, the themes that have emerged from the literature including the Gappa and Leslie (1993) categories of NTT, desired supports, evaluation, orientation, professional development, 
preparation at hire, and sociopolitical factors were used to deductively evaluate the interview transcripts. Creswell and Creswell (2018) discuss that utilizing Grounded Theory allows researchers to use both predetermined codes as well as those that emerge from the participants. Inductive coding was used to look for common themes.

The transcription of the interviews was uploaded into NVivo qualitative computer program for assistance with organization while inductively coding. Documents were also uploaded and coded for content analysis and cataloged for organization in NVivo. Corbin and Strauss (2015) discuss computer programs as helpful to qualitative researchers in organizing and maintaining data. A list of pseudonyms was maintained and utilized to protect anonymity. Artifacts were logged and coded utilizing NVivo to assist with organization and coding this information as soon as transcripts were ready.

Using Grounded Theory and constant comparison the researcher inductively coded transcripts and artifacts for themes in a systematic manner. Open coding looks for patterns in the themes from respondents (Corbin \& Strauss, 2015). Careful consideration was used to view the overall intent and theme of each interview and artifact while developing a codebook. Preliminary codes were developed by reviewing each interview for its overall meaning inquiring of the participants' intentions. Transcripts were reviewed line by line looking for patterns of meaning. This process is known as open coding and was the preliminary step in handling the data. Additionally, memos were written on each participant to ensure an overall understanding of the data. Inductive codes were then compared to the deductive codes and merged as was appropriate. Once all interviews were complete the researcher read each interview independently and wrote a memo on the interview. This ensured a constant comparison was occurring as well as assisted with not losing an individual in the group. 
Inductive coding then progressed from open to axial and selective coding. Axial coding is the process of making connections between the open codes, looking for relationships, context, or conditions upon which the phenomena appear in clusters (Corbin \& Strauss, 2015). During this phase, the researcher looked for patterns and regrouped or combined codes for axial coding. Deductive codes were used to aid in regrouping and reorganizing codes. The coding process progressed fluidly between inductive and deductive coding. For example, the code 'essential Specialists' emerged in a process of evaluating the data through the deductive code of Specialist. This code was based on the Gappa and Leslie (1993) categories of NTTs. However, as NTTs described the types of work they perform it became apparent that some NTTs held additional qualifications beyond the definition of Specialist. However, the code 'Just NTT' had also emerged showing how NTTs often felt they were treated as less than or only NTT faculty compared to their TT counterparts. The exception was specific Specialists that acknowledged that they were regarded in higher esteem and respect due to their qualifications. Dan explained why he chose the category of Specialist,

The reason specialists stood out to me a little bit was that the only reason I was hired (because I don't have a master's degree right now) was because I used to work (specific profession). It's a specialty type of career. And it's that's also a specific major of the (specific) department. So, [the reason] they picked me to do it was because of my experience, my work experience that they brought me in to do that.

Dan's identity as a Specialist was directly connected to an essential skill. The researcher then reviewed all the Specialists' transcripts inductively for discussion of additional training or certifications that set them apart. In this way, the processes of inductive and deductive coding 
were used in tandem. Several smaller ideas were also combined into a larger category. For example, 'essential Specialists' and 'Just NTT' were combined as aspects of NTTs identity. While axial coding the researcher verified definitions of codes and became aware of code drift. Creswell and Cresswell (2018) encourage researchers to constantly compare data against codes and write memos to guard against drift or wandering away from original definitions or meanings. Following methods from Corbin and Strauss (2015), the researcher looked for phenomenon, causation, and context within axial codes. These codes were used to create a codebook to reevaluate the interviews and artifacts. Once themes emerged and appeared to generate no new information the data was considered saturated. The codebook includes code definitions of the resulting themes and can be viewed in Appendix F.

These thematic codes were then appraised again, to find clear stories or selective codes. During this step graphical representations proved helpful. For example, comparing the three departments using a Venn diagram resulted in identifying clear similarities and differences. The themes that connected were grouped with arrows and brackets and eventually, a pattern emerged. Peer debriefing aiding in the clarity of patterns and themes. These analytic conversations helped to clarify phenomena observed by the researcher, such as the 'NTT network'. It is within selective coding that a theory or model emerges. In this study modified Grounded Theory produced a collection of key concepts.

Member checking was used to verify major findings. Creswell and Creswell (2018) explain member checking does not involve sending the entire interview transcript to a participant, instead, themes or major findings are confirmed with participants. As the study emerged member checking was used to allow participants to comment on findings and to aid the researcher in informing the data. Participants were emailed a summary of their interviews and 
were asked to verify that the summary reflected the interview. The researcher requested the participants affirm accuracy or reply with any corrections or changes. Some participants requested word changes that clarified the intent behind their statements. Goulding (2008) notes the importance of constant comparison, similarities and differences in cases, and core category saturation before the identification of theory. All participants verified summaries of their interviews. Additionally, participants were sent a follow-up email to verify the pronouns they wished to have used with their pseudonym to ensure the researcher was appropriately representing participants' choices. This also allowed the researcher to probe for patterns that may be influenced by the demographic of gender. The follow-up with NTTs also included verification on participants' alumni and longevity statuses at the university as these had emerged as relevant in the study. This process demonstrates another example of the use of Grounded Theory as alumni status and longevity were not originally considered relevant in the study, but later emerged as relevant during the coding process.

The researcher developed a memo book within NVivo to ensure accurate reflection for positionality and contribute to consistency, reliability, and validity (Corbin \& Strauss, 2015). A memo book was helpful with data analysis in the fact that thoughts change over time. Tracking the progress of conceptual developments early helped to observe progress in the project. The code and memo books were updated regularly. The data was reviewed in entirety to ensure the stories were intact and reflected the tone and intent of the participants. The researcher noted relevant body language, sarcasm, eye contact, and other gestures that are often lost in interviews once the words are transcribed. For example, several participants laughed sarcastically during interviews. This was noted in the transcript as 'chuckled'. This notation aided in recall when reviewing interviews up to a year after they took place. 
Negative cases were noted in memos to be considered. Corbin and Strauss (2015) outline negative cases as data that does not fit the pattern that has emerged. Such cases may be helpful to aid researchers in exploring alternative theories or explanations to a phenomenon. For example, the researcher presented probing questions to NTTs regarding supports that could aid in their feeling of value. All of the NTTs in the study responded with specific support requests, except Jason. He responded, "I mean, it's If, if I feel valued, no one can take that away from me. If I don't feel value, no one can give that to me, it needs to come from me." Jason's response demonstrates that his worldview on the term 'valued' differed from the other NTTs. Finding a negative case reminded the researcher to explore support not only as what the university can provide but also reflect on the preconceived concepts the individual NTT faculty hold regarding their value as employees and the concept of value in general. Jason's contribution in a negative case was essential in the process. Goulding (2008) cautions against rigid rules within the use of Grounded Theory but emphasizes the systematic process grown out of interpretivism that can serve to study social phenomena, such as those in this study.

\section{Ethics}

Institutional Review Board (IRB) approval was sought and acquired prior to collecting any data. The IRB process included one of the ethical standards in human research subjects and all applicable state and federal laws were adhered to. Steps were taken to ensure participants were aware of their rights, including utilizing informed consent (Appendix E). Interview protocols were adhered to, and data is represented as a whole to protect the anonymity of participants. Selecting participants from differing colleges and departments as well as using pseudonyms when reporting data assisted with anonymity. Being aware of positionality, the 
researcher guarded against commiserating with fellow NTTs on issues not related to IRBapproved protocols during semi-structured interviews. This researcher chose not to include any information that could lead directly to an individual participant and have repercussions on employment.

\section{Positionality}

Agee (2009) discusses research questions emerging from a researcher's passions and interests in particular topics. Certainly, this researcher's connection to the study lies in being a member of the NTT faculty for over 15 years. The connection of being an insider began the process of inquiry. Being an insider could be viewed as a limitation, however, being a part of a group allows for an intimate understanding of the data gathered and maybe an advantage (Rios, 2018). NTTs tend to be a tight-knit community bound by common experience. This was advantageous to the researcher in that NTTs opened up about sensitive topics such as job insecurity once they discovered the researcher was also NTT faculty.

Additionally, Berger (2015) suggests that NTT faculty should research other NTTs as insiders to explore problems they themselves have experienced. During the researcher's time as a full-time NTT Specialist at two different institutions, many of the phenomena discussed by the NTTs in this study were observed. Berger (2015) further explains that experiences can both help and hinder analysis. Corbin and Strauss (2015) discuss the interplay between researcher and participants. Having a common connection with NTTs was a benefit for the researcher in relating to participants and in coding the data into meaningful themes. However, it is of note, that themes arose that the researcher had not experienced or predicted.

Several steps were taken to ensure researcher bias did not occur and the conclusions can be considered trustworthy. First, memos were written throughout the process to record the 
researcher's thoughts and impressions. Memos assist with the transparency and reliability of the data (Corbin \& Strauss, 2015). The researcher practiced reflexivity throughout the process. This reflexivity allowed the researcher to monitor bias. Second, member checking was utilized to determine the accuracy of the findings. Verifying information and modifying interviews as requested by interviewees ensured the intent of the interviews was recorded. Third, throughout this process, the researcher had prolonged engagement with NTTs and was able to provide thick descriptions of their experiences. Such thick descriptions are indicative of qualitative studies and aid the reader in determining transferability. The NTTs descriptions were compared with those from faculty developers, administrators, and union representatives to ensure a clear picture of the NTT experience on this campus was represented. Lastly, the researcher included several peer reviewers and engaged in ongoing analytical discussions to ensure bias was not occurring. The aim was to contribute to the emerging literature of a growing group of employees while honoring their lived experience

\section{Trustworthiness and Limitations}

Every study has limitations, and these will be explained here along with a discussion on the trustworthiness of the data collected. Careful attention to the participant selection process, data collection, and instruments speak to the trustworthiness or credibility of the data. Data can be viewed as more authentic through using multiple data points known as triangulation (Ary, Cheser Jacobs, Sorenson, \& Razavieh, 2010). In addition to interviews with NTTs, this study included interviews with participants representing differing points of view and foci including administrators, faculty developers, and labor union representatives. The review of artifacts and field notes in the form of memos adds to the triangulation of the data. Multiple data sources were used to find emerging themes, also adding to triangulation. Triangulation validates data by 
using more than two sources, comparison, and crosschecking to ensure research findings match reality (Merriam \& Tisdell, 2016). Though objective truth may never truly be found, the researcher increased trustworthiness by using four independent groups of subjects to contribute to themes. Additionally, artifacts added depth to the data. Merriam and Tisdell (2016), suggest not only multiple artifacts but also approaching data with multiple theories. This process occurred while memoing and coding both inductively and deductively.

This audit trail establishes dependability in the study as others may review the decisions being made by the researcher throughout the research process. The memos served not only for reflection but also to allow the representation of progress in the study. The researcher discussed salient points with a peer assessor to ensure clear demarcation of themes. These conversations were essential and became documented memos as well.

The study is limited in the fact that its focus is specific to one publicly funded teachingfocused institution and is therefore not representative of all postsecondary institutions that utilize NTT faculty. Further, given the scope of the study, it was not possible to interview all NTTs, faculty developers, and administrators, and thus the experiences discussed will be representative of only the participants of the study. Additionally, data from the labor union was limited due to the low response rate in interview participants. However, the intent of qualitative studies is not that of generalizability. What has been lacking in the scholarship of NTTs is information as to 'why' which can best be addressed with qualitative measures such as those used in this study.

\section{Conclusion}

Using Grounded Theory methodology, this study included 23 participant interviews and a review of artifacts and memos. NTT faculty interviews from three distinct disciplines served as 
the primary data source and were triangulated with interviews including administrators, faculty developers, labor union representatives, and memos. The data were analyzed in a fluid process of deductive and inductive coding. The intention of the researcher was to code deductively and then approach the codes inductively as a secondary step. However, inductive codes quickly emerged as interview transcripts were complete. The process, therefore, was much more fluid using deducting and inductive coding interchangeably. The process culminated with open, axial, and selective coding and was represented in a codebook (Appendix F). To ensure trustworthiness the researcher used an audit trail and reflexivity along with peer debriefings. This process led to distinct themes that emerged from the data suggesting a collection of key concepts that provide insight into the NTT experience to be discussed in the following chapter. 


\section{CHAPTER IV: FINDINGS}

\section{Introduction}

The purpose of this study was to examine the perceptions of NTTs in terms of their feelings regarding pedagogical preparation to teach at the postsecondary level and the support they receive or do not receive, to do this effectively. Issues of preparedness and support of NTT faculty have grown in importance with the increase in universities using an NTT workforce to deliver undergraduate education. Chapter 3 outlined the methodology used to gather data from interviews and artifacts. Chapter 4 presents the analysis of the data based on participants' answers during the interviews and the documents reviewed. The discussion will include the emergence of key findings that surfaced as themes were identified within the data. These findings emerged as a result of using Grounded Theory methodology to answer the following research questions:

RQ 1: How pedagogically prepared did NTT faculty feel when they began teaching at the postsecondary level?

1a. What types of previous experiences or pedagogical training inform NTT perceptions of preparedness?

RQ 2. How do NTT faculty describe the pedagogical support they receive in their teaching roles?

2a. What is the role of professional development in supporting NTT faculty teaching?

2b. How are evaluations used to inform NTT faculty teaching and retention?

2c. What additional supports do NTT faculty desire to improve their teaching? 
The research questions were used to analyze the data. The software program NVivo aided in the organization of the 23 interviews and available artifacts. This information was first deductively coded for themes based on the literature. Next, an inductive process of open, axial, and selective coding was used as is described by Corbin and Strauss (2015). Constant comparison was employed at each step to further sift the data until clear themes emerged. A codebook (Appendix F) reflects code definitions. The resulting themes that emerged from the data give voice to the NTT experience and provide answers to the research questions posed. Using Grounded Theory four primary themes were identified. These themes include the NTT network, identity: things aren't always what they seem, evaluations: what's the point and value: it's not about the money. Insights from each of the research questions will be discussed including visual representations and quotations from participants.

\section{The NTT Network}

The researcher defined the NTT network as informal sources of support that NTT faculty sought from friends, proximity, or colleagues both TT and NTT faculty. There was a phenomenon observed where NTTs who are not provided clear expectations, onboarding, assigned mentors, or information on evaluation and professional development seek out this information from a network of friends, coworkers, and other NTTs to acclimate to the university environment. NTTs in this study referenced the importance of colleagues and connections that were created informally to gather the needed information regarding onboarding, evaluation, professional development, and integration into campus culture. While the university does have orientations, these are optional for NTT faculty who are often unaware of their existence or unclear if the orientations are intended for TT faculty only. Lacking formalized support such as

procedural and cultural expectations of their departments and assigned mentors to ask questions, 
the NTT faculty created their own networks of connections to aid in acclimating to the university and their roles on campus. These informal NTT networks are forged from necessity and take the form of friendships, proximity, chemistry, self-reliance, and a bond formed from being in a similar situation. Administrator Alex explains the informal way NTTs are trained for their roles, "I think a lot of that is passed on sort of indirect". Within this theme of the NTT network is the relational connection to others that was important to supporting new NTTs. Gretchen, a full-time Aspiring Academic explained the importance of informal relationships, As NTT's we don't really have a faculty mentor like a lot of the TT faculty do. So, you kinda just have to rely on asking others for understanding things like you know how does getting your contract signed work and you know how to things like getting status work and some of those other pieces.

Gretchen is describing relying on colleagues for information and guidance in many aspects of her position. She mentions procedural, evaluation, and relational support that are components of being a member of the faculty. The NTT network is used to aid NTTs information needed in the performance of many aspects of their positions.

When digging deeper into the NTT network the researcher found five unique ways the network is formed. First, NTT faculty reported feeling like they need to help other NTT colleagues because they were lost when they were hired. There is a giving back to the group that occurs. You were new once and an NTT faculty member helped you, in turn, you will help the new person to acclimate. Second, taking the initiative and asking. The NTT faculty who doesn't have a co-worker in the office by proximity or preexisting relationships need to take the initiative to ask questions. Third, NTT faculty have friends that already work at the institution. These friends are from a personal network from living in the community and knowing people that work 
on campus. They rely on these friends, regardless of the department they work in to be primary sources of information. Fourth, there is a chemistry of just clicking with someone and feeling comfortable asking questions. This comes from being willing to help one another and having an affinity for the person. Some people just get along well with one another. Once they have developed a bond, they continue to go to that person for information and departmental questions. Fifth, proximity, where your office is located or who you run into in the hallway by nature of your schedule, you ask these people for help. This is like becoming best friends with your neighbor when you were a child. It's merely a relationship of convenience based on location. NTT faculty are succeeding in integrating into the campus despite not having formal supports. Acclimation and integration are occurring through informal networks. However, the NTTs, administrators, and faculty developers also described a variety of formal orientations and onboarding opportunities that will be described next.

\section{Don't They Have Orientations?}

Optional orientations are offered at the university level organized by the Faculty Development Center, Benefits and Human Resource offices. Additionally, one department held an optional orientation specifically for NTT faculty. The University Faculty Development Center offers an orientation in August for new faculty. The orientation introduces attendees to the mission of the university, demographics of the student body, and resources such as the Faculty Development Center, the library, and the course management system. This orientation also includes a welcome reception of all new faculty at the university president's home. This program is open to both TT and full-time NTT employees. While the NTT that attended this daylong training found it helpful, others did not hear of the orientation due to being considered parttime their first semester hired. Some NTTs felt the orientation was meant for only TT faculty 
due to the title using the term faculty and did not attend. The term faculty is sometimes used to mean both NTT and TT faculty and at other times is used for TT faculty only. Thus, if NTT faculty are not specifically mentioned, it is unclear to the NTTs if they are welcomed.

Additionally, NTTs may be hired late into the summer after invitations have gone out for the orientation or are hired for the spring semester only and completely miss the orientation. Dan is a Specialist that teaches part-time in two departments enough courses to be a full-time NTT faculty member. Dan's contracts vary from no courses some semesters to full-time course load in other semesters. He shared a time when he was called in only 3 days before the semester started, obviously being unaware of the faculty orientation.

The representative for the labor union explained the orientation is standard and intended for all faculty, but some NTTs may be excluded as it is the responsibility of individual departments to communicate the availability of this orientation. The faculty developers themselves agree that NTTs are a difficult group to ensure they are included in orientations, especially part-time NTTs as departments may neglect to include NTTs on the invitation list. Further, as Dan explained, he chose to not attend some meetings his first semester hired as he was unsure if his appointment would be a 'one off'. Essentially, unsure of the length of his employment, orientation did not seem necessary. NTTs that are unsure if they will be hired for more than one semester may not find it a valuable investment of their time to attend training for a job that appears limited to one semester. Further, NTTs that work jobs off-campus, such as Freelancers and Specialists, may be unavailable to attend orientations due to schedule conflicts with outside employers and not attend an optional session. These orientations were only available in person until the Covid-19 pandemic. As the study took place during the pandemic, faculty developers acknowledged that not having orientations available in online formats was 
exclusionary. The university plans to provide online orientation modules in the future that will aid in minimizing the impact of schedule conflicts.

This is a similar finding to the benefits orientation. Benefits orientations are available to the NTT faculty, but due to the nature of how full and part-time appointments are defined, some NTT would not have access to the information. Karen, a faculty developer in the Benefits office explains that for university benefits there is a clear definition of full and part-time based on benefits-eligible including health and retirement. Essentially, in a fall semester, NTTs teaching a $100 \%$ contract would be eligible for benefits and thus contacted to attend the benefits orientation. This is referred to as full-time equivalent or FTE. Karen explains the complexity of determining FTE,

Where it becomes complicated with the NTTs is how we average them. So, if you hire on in the fall, but only on a four-and-a-half-month contract, we have to take the FTE of that contract and divide it in half, because you're only working half of the normal academic year. So, if you are 50\%, you feel like you're a 50\% employee. But if your contract is only for one semester, you're only $25 \%$.

This arrangement appears logical, if you will not be eligible for benefits, you would not be offered the information. The difficulty lays in the fact that information provided at this orientation goes beyond that of just benefits. While the part-time NTT would not be concerned with insurance offerings unavailable to them, they are missing out on information pertaining to wellness, parking, campus recreation, and other items that assist in acclimating an individual to the culture and resources of the university that is discussed during the benefits orientation. Additionally, department administrators may be assuming all NTT faculty receive this 
information at orientation. One administrator, Casey, responded this way when asked about NTTs orientations,

They typically have an orientation in the office just in terms of the facilities. They participate in the new faculty orientation at the university level.

All three administrators interviewed assumed the NTT faculty in their departments received information from a university-wide orientation. However, as described above that is not the case. Part-timers, Freelancers, and Specialists that work off-campus and those that were not made aware of the orientations as well as those hired spring only may not attend the university orientation. Excluding part-time NTT positions could be problematic because many of the fulltime NTTs in this study evolved from being part-time. Administrators may make the false assumption that the newly converted full-time NTT faculty member was trained during their part-time NTT appointment.

During the research, it was discovered the university offers an optional acculturation orientation to full-time employees using the FTE definition. This means, that an employee may not be considered full-time to attend a benefits orientation but could be full-time and thus invited to an acculturation orientation. This optional orientation begins with the university's history and then proceeds to explain the culture of the institution. Faculty developer Tyler has been presenting these sessions for several years and described the orientation as somewhere between a TedTalk and a PowerPoint. This orientation includes details on the universities mission, vision, and values. Tyler is enthusiastic and passionate as he rapidly explained the importance of feeling connected to the university. The orientation allows for small group interaction through shared activities, videos, and connections to campus including Q \& A. The group discusses ways they have seen their office demonstrate individualized attention or ideas they have for demonstrating 
individualized attention. Other components that allow for group cohesion include eating in a student dining area, photos with the mascot, and videos that showcase students. Participants have opportunities to process meanings of each component of the orientation and to be connected to the content. Tyler estimated that about $10 \%$ of NTT faculty attend. The NTTs in this study stated they had a desire to gain insights into the cultural expectations and sociopolitical nuances of the department in which they will be teaching, however only one person had attended the acculturation orientation.

The labor union contract references orientations (Agreement, 2017) stating the department will provide information about operations, procedures, and expectations within the first 30 days of employment. This orientation is to be at the departmental level and the labor contract specifically states this as separate from the human resources orientation. Of the three departments interviewed, one provided an NTT faculty-specific orientation that could be viewed as including operations, procedures, and expectations. The other two departments have no formal departmental NTT faculty orientation. All three departments referenced office support staff that assist NTTs in finding mailboxes, photocopies, and other procedural items and provide directions in wayfinding for the building. Department B offers departmental NTT faculty-specific orientation in the fall including information on departmental philosophy as well as practical information such as library use. The optional full-day event called the NTT kickoff includes 1520-minute presentations from campus entities designed to provide information that supports NTTs' teaching practice. The NTT faculty that attended this orientation agreed it was extremely beneficial to aid in their acclimation and adjustment to their department. Full-time Freelancer Maria explained that she can tell a difference between herself attending the optional orientations and her friends that did not. 
I felt like that it really helps understand helped me get at the idea of the culture and all that and if I didn't go to that I would've been lost and I have friends that chose not to go to that, and they seek me out for information that I learned at those orientations.

While Maria sees the benefits, the departmental orientation is new and seasoned NTTs voiced that they have been on campus long enough to acclimate on their own. Maya is a part-time Freelancer who has worked for three different postsecondary institutions for approximately nine years. She explained she also felt it was unnecessary to attend if she was not going to be teaching.

I did not go to it because when I look at the agenda, I saw at that point in time I've been teaching there long enough I uh oh I have known you know like I knew some of the answers or I know that was one time I didn't go and another time it was when I wasn't assigned to a class. Uhm, I didn't really want to go to this training because this particular semester I'm not assigned you know a course.

Maya's comment demonstrates that it is difficult to be motivated to invest time in training for a position that may only be temporary. NTTs hired in more recent years have more opportunities for orientations available. Orientation experiences among the NTTs interviewed varied greatly from none to office tours to multiple in-depth sessions. The differences lay in the individual departments, the year the employee was hired, and in their full or part-time status their first semester. The commonality among the NTTs was a desire at hire to acclimate and be included in a new environment. Jason is a Freelancer that has taught full-time NTT for approximately 20 years at multiple institutions. Jason's response is representative of NTTs that did not receive orientations. 
I never sat down for a here is what you need to do. I never had any sort of orientation or mentorship as far as university procedures. No, no, nothing. No, whenever everyone is just, it was assumed that I would seek it out as I needed it, which is a little odd.

Jason characterizes his experience as odd, however, this was a common experience mentioned by many demonstrating a feeling of isolation. They were on their own to learn their place in the department and on campus. Sara is a full-time Specialist with over ten years of teaching experience. She is one of the NTTs that received a tour of the departmental office for orientation but wanted more information and onboarding.

I mean I feel like there was here's your mailbox or here is this but like I remember I didn't know where to get scantrons or you know like a lot of things you just figure out along the way, but even if they now have a little orientation when I started that was not there so I felt like it was a lot of trial and error (chuckles) for what you should be doing Failure to participate in new employee orientations due to being omitted, scheduling conflicts or, opting out NTT faculty acclimate in other ways, this is how informal networks are formed.

\section{Lead Instructors/Program Coordinators}

All three departments used lead instructors/program coordinators to oversee subunits or specific sections of courses with similar content. These individuals are assigned the duty of coordinating courses taught by NTT faculty and have some level of formalized curricular support to the NTTs. The NTTs do not necessarily view the assigned colleagues as supervisory, but more as midlevel managers. Lead instructors/program coordinators are TT faculty that are receiving additional compensation or release time for the time and effort to coordinate the courses and the NTTs that teach them. It is of note that in all three departments the program coordinator/lead instructor had no direct responsibility in evaluating or hiring the NTTs. From this perspective, 
the role of the coordinator/lead instructor appears more curricular in nature than managerial. While this allows the program coordinator/lead instructor to focus on course content it can be confusing for newly hired NTTs to know who to go to for questions.

These individuals are relied upon by administrators to acclimate the NTTs to their positions. This entails providing past syllabi, textbooks, and if applicable required assignments. However, there was no clear training or expectations for the program coordinator/lead instructor and thus the type of support provided varied greatly among and within the departments. Some NTTs spoke of an ongoing connection and bond others however did not experience the same support and mentorship. As mentorship is not a requirement for the program coordinator/lead instructor, feeling welcomed and having a safe person to ask questions of is not guaranteed for all new NTT hires. Jason had assigned colleagues that provided only the basics of information. He shares his story of minimal guidance.

what support did I receive? Um, I had a course coordinator that said here are the here is what you here is your book. Here's what you need to cover, we're going to make a final exam. Here's what the final exams used to look like, prepare the class for it. And that is pretty much the extent of support that I got.

Jason's experience is not one of social connection, mentoring, bonding, or ongoing support. Instead, he demonstrates the procedural aspect of being given baseline tools to complete the task. Contrast Jason's experience with that of Maria.

My very first class at (University) I felt very prepared, but I do believe that was because of the specific lead professor for that course she made an appointment to meet with me and really was there for me all the time. She made sure of that. She was very proud of the course that she developed, and she wanted it to maintain that high standard. So, she 
really went above and beyond to make sure that us as NTTs were able to deliver the course material up to her standards.

Maria mentions that the assigned colleague not only provided materials but also had a meeting to ensure communication was clear and course delivery was consistent among the NTTs. This finding suggests that NTT faculty with assigned support colleagues that meet with them and are available in a mentor role versus a minimal role feel more supported and connected. Lacking consistent formalized support, the NTTs rely on a created network to meet their needs.

\section{"It's NTT Teaching NTT"}

The most often mentioned informal NTT network was NTT teaching each other. NTTs demonstrated a feeling of connection with one another as they can relate to being new in an environment with limited information and communication as to expectations and orientation. Repeatedly stories arose during interviews of NTTs who felt lost and alone and turned to the empathy and understanding of other NTTs to help guide them. The fraternity of NTT faculty goes beyond casual co-workers and is akin to being in the trenches with an ally. It is the connection of people who think in a similar way and understand each other well or can demonstrate empathy for someone's plight because they themselves have 'been there, done that'. This phenomenon has a long history. The researcher's positionality of being an NTT faculty was advantageous when subjects were hesitant to explain a feeling or experience. The researcher would mention understanding a similar experience from their own employment. This affiliation would ease the conversation and allow the NTT participant to relax and share. The kinship of sharing a similar lived experience facilitated disclosure during NTT interviews.

Additionally, for NTTs that have not yet acquired status, there is a vulnerability because they may not receive a contract from semester to semester. With job instability, NTTs are 
cautious about what questions they ask and to whom. The NTT faculty kinship was demonstrated during the interview with Maria. Maria was meeting with a friend who had just been hired into a part-time NTT position when we spoke on the telephone.

Like I just had a girlfriend come over here actually right two hours ago that just got a job as an NTT and I'm sitting there filling in the pieces for her on my free time. But that's what I wanna do. But I feel like it's a lot of us being collaborative on our own, if that makes sense. I mean she is over here for three hours, and I was over at someone's house two years ago consecutively teaching about the job. I think a lot of it is NTT's teaching each other sometimes.

Maria felt prepared to teach due to another NTT faculty member investing their time to ensure information was communicated clearly on expectations, orientations, procedures, and policies. The cycle continues as she is helping a friend and newly hired NTT faculty acclimate. Latoya a Specialist with 29 years of experience as a full-time NTT described the bonds of NTT faculty helping one another.

Well, I think there's sort of an indirect, like I said, mentoring system that goes on. I don't know for sure that, you know, that departments do it, you know, like, officially, but unofficially, non-tenure track have been assisting each other for as long as I've been there. So, we almost find it as part of our job that we help out the new people. Now, like I said, that's not real official.

The connection of NTT faculty helping one another is deep and has been happening for as long as Latoya can remember. Having been employed for 29 years that she explained this as a significant amount of time. She described the support as not official but necessary for acclimation, connection, and information. Juanita's story is just as compelling. Juanita is a 
Freelancer that typically teaches at two or more institutions and has done so for twelve years. She told of a small group of NTTs that work together not only in orientating one another but also in creating a mentorship and support network that continued through their employment. She explained their bond is not only through chemistry (they just like each other) and proximity (their offices were located next to one another), but because they all identified as being NTT faculty.

I can't say that it was anything (the university) did for me. We just had our own little group. And it was a unique group of individuals and that we all help each other. There wasn't anything anyone needed... We also legitimately like each other and spent time with each other outside of work. And yeah, it was more It was a very much a family atmosphere. So, you didn't feel like you're on your own with anything really.

She attributed her acclimation and ongoing support to this unique group of NTTs and not the university. The support of this group of NTTs may not be as unique as Juanita thinks, in every department NTTs shared similar stories. NTT faculty help NTT faculty. That is not to say NTTs do not utilize their TT colleagues or administrative supports as well. It was s just more commonly mentioned in this study that NTT faculty help other NTT faculty. Gretchen felt this was due to the lack of a formal mentor program for NTTs. She explained that NTT faculty support each other in many aspects of their positions such as information on job security, continuous appointments, evaluations, and procedures.

Thankfully we have a lot of NTTS in our department who are vocal and well organized and understand how the process works and so speaking to multiple people in our department and understand how the process works and so speaking to multiple people in 
our department and of course, our school directors have been very helpful and very hands-on and the open door for questions about that.

Gretchen described the NTT network as organized and vocal in helping other NTTs. Information is passed among NTT faculty in departments in a tight network. Gretchen is not referring to an organized labor union, but rather a much more informal arrangement. Once NTTs are aware of a new hire, other NTTs go out of their way to ensure department-specific information is communicated. This information may include negotiated rights such as how to obtain status but may also be as innocuous as how to dress in the office or where the microwave is located. NTTs share information with one another on departmental culture regarding expectations of attendance at meetings and social events as well as information on professional development and evaluations. Maria wishes she was given clear expectations for her position and explains that as a practitioner she would normally have a supervisor and clearly directed job duties.

You know in the profession your supervisor would say this is what you do, and you do it. And then here I like the freedom, but I do think like what is the expectation?

The NTT network and context clues are used to determine the expectation of the position that are not explicitly stated. For example, office hour requirements are not explicitly outlined in any of the departments in this study, NTTs establish these based upon comparing with one another. Isabella has been a full-time Specialist on and off for six years. She was concerned that information communicated through the NTT network may not be accurate or consistent. She said much of the learning about departmental expectations just happens as you do the job but felt orientation and integration for NTTs should be more formalized. She remembered her onboarding process, 
I don't think I ever got anything. It was like hey these three things need to be on your syllabus. Like none of that has ever come to me formally everything has always been an informal conversation with other NTT's (chuckles) so like that, I think could be explained a little bit more.

The researcher found that NTTs seek and provide support to and from one another creating an informal NTT network that serves as a source of onboarding, procedural information, mentorship, connection, and ongoing resources. These supports are desired by the NTTs and currently are not being formally offered by the university. Lacking formal mentorship and onboarding processes the NTTs have created their own methods to fill the gaps.

\section{Seek It Out or Be Lost}

NTTs described having to seek out information independently acclimating and integrating into the environment through observing behaviors, asking questions, or independently exploring sources of information. Maya had been teaching at several institutions but was trying to find information specific to the procedures at this university.

However, specific to (University) I thought I had to do a lot of seeking out of information. At the time when I started teaching [at the university], I was also a doctoral student, and I was also working part-time, so I knew some (emphasis on the word some) things about teaching but a lot of it is program specific uhm or even like department specific so I did feel like it was a lot of me having to seek that out in a lot of it. I sought it out because I knew how it was at other institutions as I was looking for a similar answer to questions I might have.

Maya demonstrated how she was learning from her experiences in teaching at other academic institutions, by seeking similar types of information at this university. In addition, she homed in 
on the differences between departments, acknowledging that learning one specific department culture or expectations may be different than acclimating to the campus as a whole. University culture is conveyed through policies, priorities, and public statements such as the mission statement. Each department also has a unique culture, established procedures for evaluation, attendance at meetings, expectations for social gatherings and a myriad of other procedural and social expectations. Having to seek answers to procedures that could easily be communicated at a departmental orientation or meeting is time-consuming and frustrating. Twelve of the NTTs interviewed referenced having to figure things out on their own. Lacking formalized orientations or mentors for continued support sends a message that the NTT faculty are not valued enough by the department to have ongoing support. At three different points in the conversation, Maria mentions self-discovery.

Because I did, I did kind of had to teach myself that and try to seek out more information on this in the summer, you know do my own research. I felt like that was more me my own research.

Second mention

Ijust do think you have to have motivation and initiative to do things on your own time or you could be really lost.

Third mention

But it's almost like nothing formal. It's kind of just informal conversation. I'll find out about things in meeting people but again it's a lot on my own it's not told to me. I think you could be really lost if you didn't you know seek it out for yourself.

Maria explored both pedagogy and procedure. She sought out individuals and independently researched answers to her questions. Maria felt you could be lost without motivation to seek this 
out on your own. Gretchen used this same term as Maria, "lost" when describing her lack of understanding. She adds in a component of trying to learn the specific pedagogical cultural expectations of the university. Certainly, a more nuanced support need than procedural information.

So sometimes I am a little lost and I have to catch myself up, but it's a learning experience. I think just asking questions and being involved and being cognizant of what you don't understand has been the most helpful. For me, it has been trying to zone in and see what (University) expects and wants of their faculty.

Gretchen shared that her learning her role at the university not only comes from experience but also in decoding the tacit assumptions present. NTTs are attempting to learn procedure, cultural expectation, and curricular information all from informal sources. Jada mentions the curricular process as something she had to learn about on her own. She was not aware of universityapproved syllabi and approved content used for assessment.

But I had no idea (emphasis added in increased volume) about that. I didn't know about the curricular process and how that worked. No one had ever told me about that. You know you can change this this and this, but you can't touch this. I didn't didn't [sic] have a clue and so I felt kind of like seriously wow. Now no one's ever said that. Shouldn't that be something that should be, maybe not somebody who's the first semester, you don't know if they're going to stick around kind of thing, but somebody has been teaching for so long, you would think, I guess. I had thought maybe that something that should be communicated but (long pause) yeah really didn't get much of anything other than here is the course, have at it. 
Jada felt the curricular process should be communicated after NTTs are committed to teaching multiple courses to ensure NTTs are aware of the assessment expectations. Specifically, she was concerned with content that could or could not be changed within an individual course. Jada learned the process and has successfully contributed to her department's curriculum by proposing course changes and new course offerings. Jada and two other NTTs like her were actively involved in the curriculum. Contributing to the curriculum is a way for these NTTs to be included in the department as experts in their profession, adding to their sense of value and connection. The NTTs felt contributing in this way also provided evidence to renew their contracts. Curricular expectations, pedagogy and departmental culture are all things that NTTs seek on their own through the informal NTT network.

\section{"I Have a Friend"}

NTT faculty interviewed explained that they had friends on campus either in their department or in other departments that they could approach for assistance. Assistance ranged from procedural items such as where to make photocopies and get textbooks to pedagogical items such as how individual assignments are developed. Additionally, friends provided insight into departmental expectations, evaluations, professional development, and campus hierarchy. There is a comfort level in asking a potentially obvious or embarrassing question of friends that have an established rapport. The friend may be an acquaintance, an individual with a close relationship, or someone known through social media. The preexisting nature of the relationship was what was important in seeking answers.

Maya is a Freelancer with a background in teaching. She teaches for multiple institutions and in two departments at the university represented in this study. She explained the informal nature of the support she received from her relationships with current university employees. She 
shared that she could lean on the people in her department and often does for information such as curriculum details and individual course expectations. These friends were viewed as allies in discovering the required competencies for the students she teaches. Maya compared her experience to the other institutions she works with and found at this institution she relied heavily on friends for information. She felt she had to do a lot of reaching out to friends rather than having information presented to her by the university.

Similarly, Juanita teaches at least two different institutions as a full-time Freelancer for the past 12 years. In Juanita's opinion, NTT faculty are an economical and flexible way for the university to have a variety of teachers. Juanita was prepared to teach by having a teaching degree, past teaching experience, and an informal network of coworkers. It is her connections that helped her to initially acclimate and continue to grow in her role as a member of the faculty. Juanita had no formal orientation but knew people on campus and could go to them for assistance. Juanita says she just knows who to go to if she needs anything, purely from having friends.

The friendship connection is not unique to Freelancers. Marcus is a Career Ender with a pedagogical degree and experience in the private sector. He started teaching in postsecondary education last semester. He felt most of his integration to postsecondary education came through administrative email and friends he knows in several departments across campus. Marcus mentioned that he had no formal orientation but emphasized his connection to the university through friends. He explained he would go to his friends for support first, then the office staff, and finally the administrator. He has established a hierarchy to meet his support needs starting first with his friends. His words convey his experience, 
I have several friends who work here in the (specific) department in (specific) department and stuff...Uhm probably first professor (the name of a specific friend in another department) then probably (name of another friend) just cause he's been here longer than anybody else I know. I would also check with the (assistant director) or (administrative support). Those those [sic] two people are the key people here beyond friends.

It is the informal knowledge of faculty that have been on campus longer than anyone else Marcus knows that is significant to him in finding information important to performing various aspects of his position. All three of these participants demonstrate that having a friend as a known connection was an important part of their onboarding and integration processes. The friend may be TT or NTT faculty, casual acquaintance, or close relationship. NTTs are not assigned mentors to help answer questions in procedure and pedagogy and as a result, they find connection and comfort in friends. Other NTT faculty find a co-worker they can relate to by means of an unspoken connection.

\section{"It's Kind of Up to The Chemistry"}

There were cases where a friend was not in place in the hiring department or across campus, and the NTT faculty member meets someone who matches their needs, influencing them positively. Having chemistry means a mutual connection or affinity for one another. Jada a full-time Specialist with over 20 years of experience feels fortunate to have the support of a colleague that she says just "clicked" with her. Receiving no formal orientation, this connection was influential in her not only modeling her teaching practices but also in feeling valued. She stated it was this one person who took the time to invest in her and help her and that made all the difference. 
He was just the most incredible uhm resource and support of anybody in my professional life. So that for me I think it's probably being blessed to have worked so closely with him, it's over these years having his encouragement and modeling that has probably taught me more so much more to help me to learn about service opportunities for uhm and you know those kinds of thing.

The human connection of a colleague being encouraging and available to assist with questions was influential to a positive acclimation and integration to campus. Jada was able to get guidance on what departmental committees to serve on and pedagogical methods worked well in her curriculum through observing her colleague. This support provided connection and contributed to her sense of value. Administrator Pat explained connecting with colleagues may be an orientation of chance. Lacking a formal departmental orientation, this department administrator relies on faculty getting along well enough to be willing to help with acclimating NTTs to the campus.

Ifeel like it's kind of up to the chemistry that might evolve between the NTT and other faculty members.

Pat was hoping that the NTTs would connect with someone as a way of integrating into the environment. Many factors determine a positive onboarding experience for new employees at the university. NTTs in this study cite a need for human connections to provide insights into campus culture, policies, procedures, and pedagogy. Those that did not have the support of assigned colleagues through chemistry, seek to fill that gap themselves through other aspects of the NTT network. Another haphazard way NTT faculty met their supports was by having an office in a similar location. 


\section{"So, Where My Office Is Located"}

NTTs receive support not only from coworkers through a connection but also with those that they share space. This aspect of the NTT network is generated by proximity. NTT faculty are in the right place at the right time, rather their offices are. The support is informal and emerges naturally due to proximity or relational connection. Gina, a full-time Aspiring Academic with a pedagogical background felt there was not much guidance at hire. She recalled the location of her office as what was most helpful.

So, where my office was, is located. There's a hallway, all NTT's and I had an officemate, and so she had been teaching at (University) for several years at that point. So, she was kind of my she can help me out navigate that.

Gina relied on an officemate that she met by coincidence. She is not the only one that was fortunate in her office location. At least one NTT faculty in every department studied found support by office location. Gretchen laughed at the fact that her support came from location, saying she could just ask the person across the hall. Gretchen explained that NTTs do not officially have mentors and the fact that she could ask a colleague located in close proximity to her was the most helpful to her onboarding. Her laugh has a tone of sarcasm highlighting the lack of planning on the part of the university to provide NTTs with formal mentors. Gretchen's officemate provides her with information on how the NTT process works on campus including navigating status. Dan also shared office space with another faculty member. However, in Dan's case, the individual had been his instructor when he was an undergraduate student 25 years ago. By way of sitting next to this person, he is more comfortable asking questions and receiving mentoring assistance. Dan finds it a "weird" coincidence. Fortunately, Dan and Gretchen were located near a colleague that was both available and willing to help. However, it begs the 
question of how NTTs that teach in the evening or have offices tucked away from everyone or no office at all are mentored? Some NTT faculty are not lucky enough to have a desk or office located near someone willing to help and must seek information using other aspects of the NTT network.

While all jobs have components that can only be learned with experience, NTT faculty positions at this university are unique in having no standard orientation, no formalized mentorship, no guarantee of continued employment, and often unclear expectations and evaluations. Lacking information, all the NTTs interviewed used some aspect of the NTT network to navigate various aspects of their positions. As much of the information received is through informal sources, NTTs may or may not be garnering accurate information.

\section{Identity: Things Aren't Always What They Seem}

The second major theme was about identity. Identity encapsulated how the NTT faculty participants perceive their role at the university as well as their social identity, or how others perceived them. Role identification is enmeshed in a complex sociopolitical web impacting the experience of being an NTT faculty member. When exploring participant statements that centered around tacit assumptions about workplace expectations, observed social norms, feelings of value, inclusion or exclusion in the workplace, social hierarchy, and other sociopolitical factors it was determined that these types of experiences had impact on position identification, termed by the researcher as identity. The narratives illuminated the sociopolitical factors that demonstrate power dynamics that can be difficult to describe to a casual observer. This is due in part to the multi-layered aspects of social environments. The theme of identity: things aren't always what they seem, included such things as the language of Just NTT, the role of NTTs, the distinction of essential Specialists, alumnus of the university, and representation. This theme 
highlights differences and similarities among the NTTs in all three departments and includes perspectives from faculty developers, NTTs, administrators, and memos from the researcher. The Identity of Less Than: "Just NTT"

The most prominent factor in NTT identity that was discussed by NTTs and administrators alike was the term 'Just NTT'. This term included statements to minimize or devalue the position of NTT faculty as less than their TT counterparts. The language of 'just' had a connotation of subordinate in a university hierarchal system. A cultural stigma exists that regardless of the degree obtained or the status achieved, if a faculty member is not in a TT position, they are less important at the university. Jada is a prime example of this phenomenon. She holds a terminal degree and considers herself a Specialist but voiced frustration that despite her advanced degree she cannot obtain respect for the position of NTT faculty. Jada shared how her colleagues' perceptions of her position impacted her,

Do you know, I just kind got frustrated because a dear friend of mine, who's a colleague, told me you know there's people that I'm working with that will never see me as anything but a non-tenure-track... I had applied for that tenure track position that came up and I wasn't even offered an interview... and you know that that hurt beyond words.

Jada was hurt by those in her department that did not respect her position due to the title. She felt she was overlooked for a TT position because she had been in an NTT role for so long that she was now labeled as less than. Jada shared that she believes the work of the NTT faculty is valuable in contributing to the university but lacks respect from administrators and some TT colleagues. Jada did go on to say that some of her TT colleagues value her position, reflecting that both position types (TT and NTT) are needed and equally important. The NTTs and 
administrators interviewed shared a range of interactions between TT and NTT faculty on a continuum from assistive to exclusionary.

How TT and NTT faculty interact appears to be an individual choice that is influenced by an established university system that reinforces a social hierarchy of separatism. The social hierarchy was established from the origin of NTTs as temporary workers, often equated with having less expertise. Otherwise, they would have been hired as TT faculty (Umbach, 2007). The researcher found that NTTs are no longer used just as temporary workers, the times have changed. Further, given the lack of acclimation and integration support the university system hasn't caught up. But like so many social structures the beliefs and practices remain long after the change has occurred. NTTs in the study repeatedly mention the desire to be viewed as equal colleagues. Maya explains,

I think it would just be like being seen as like an important part of the department...I feel like we're seen as like only NTT...so if it just feels like we're kind of on the cusp of things. We often get our assignments later...so it just feels uhm sometimes like we have like no representation or no say in things. Uhm and so I guess I just I feel like were viewed as important to keep the University afloat, but we are definitely not always treated that way. And so, I I [sic] wish that there was more of a collegial view like even though I'm quote end quote just an NTT ...uhm but I do wish there was just like we were just seen as like also important members of the University community.

Maya provided some insight into the dichotomy of public and hidden transcripts. On the one hand she is being told she is important in a public transcript but experiencing hidden transcripts of having limited power and voice. She is not alone in this dual nature. NTTs in this study often characterized their experiences by saying things like "I love it here, but...". The NTTs would 
explain how much they loved the students and teaching and are grateful for the opportunity to teach and are passionate about the job but were frustrated with the lack of recognition and input, position instability, unclear expectations, and low position of the NTTs. Statements of pleasure and displeasure of the workplace, vacillating or mixed emotions are common in the NTT interviews often within the same thought or sentence. Language is used by the NTTs that demonstrates simultaneously loving the work and being disappointed in a system that devalues their contribution.

The identity of less than does not stem exclusively from interactions with administrators and TT faculty. The NTTs themselves describe their position as 'just' or 'only' implying a less than or simply NTT faculty. The language stems from the title itself of not being tenure-track. However, the term 'just' or 'only' serves as a diminishing adjective. NTTs in all three departments described times they felt they do not have a voice, or their opinion doesn't matter either within their departments or in campus leadership. This lack of input is coupled with a fear of repercussion due to the lack of security in their positions.

NTTs are often a vulnerable group due to the precarious nature of their positions which contributed to a reluctance to push for change (Kezar, 2013a). During the study, several participants voiced concern that information provided in their interviews could somehow be linked back to them. The researcher reassured the participants of the steps that would be taken to ensure confidentiality. Confidentiality concerns were voiced with phrases such as, "when you use the research my name isn't in it "and “...is this anonymous?" These statements were tied with fear of job loss, lack of security, and instability. Participants would follow up with stories of desired supports or concerns that they wanted to be included in the study but did not want to be linked to them personally. This is evidence of the power structure present within the university, 
with NTTs falling at the bottom of the sociopolitical ladder and another component of being 'Just NTT'. One participant shared deep insights into the departmental hierarchy and later contacted the researcher post-interview with concerns of repercussions. For their anonymity, the participant's name will not be included here. The following memo was written by the researcher that day and conveys that the participant is both afraid and reconciled to their situation.

When you get comfortable in an interview and share the hierarchy. The meanness that has happened to you. Being treated poorly for just being you. But then you call back and say, "don't tell anyone I said that It won't change anyway." It sucks to be treated poorly for just doing your job. For just being. How can this ever be valued and important work if it's not valued in its own right?

Several participants were afraid that speaking up would be discovered and impact their ability to have future employment. Evidence of this insecurity was emphasized when the researcher began searching for interview participants. One department produced no respondents until the administrator contacted their NTTs themselves, essentially granting permission to be involved in the study. The fear of repercussion and precariousness of the NTTs position is ever-present in the NTTs identity.

Additionally, the researcher contacted each participant with a summary written from the transcribed interview for the purposes of member checking. Several participants asked for sections of their interviews to be deleted for fear the information could be connected to them. The researcher removed the requested information. However, the fact that participants do not feel they can speak openly should be considered closely along with the labor union's unwillingness to participate in the study. This information leads the researcher to be reminded this is a vulnerable population. It further gives evidence to the importance of this study to give 
voice to the NTTs that lack the power to change their circumstances in an environment that functions in an antiquated hierarchical system that perpetuates that culture and identity of less than.

In addition to a fear of transparency, NTTs in all three departments felt at times being 'Just NTT' meant they lacked prestige, value, or real power to influence decision-making at either the university or departmental level. Gina gives her insights, So, I feel like for the most part, at least in our department, the tenure line has a level of respect for the non-tenure line. But it's very obvious in some cases that they that there's a level we're just non-tenure track. We just have a master's, or we didn't weren't able to get a tenure line position for some who who [sic] do have a Ph.D. And the decisions that are made are ultimately made by the tenure line, not the non-tenure line. We might have a voice but generally speaking, we'll hear you out and then we'll make the decision.

Gina's story demonstrates the label assigned as 'just'. This term can be used interchangeably with only. As Gina astutely points out some of the NTT faculty have terminal degrees, but by not having a current TT position their input is regarded as less relevant. Latoya explains the hierarchy pointing out that NTT faculty realize they are different than TT faculty. She shares that she believes there have been improvements in acknowledging the contributions of the NTTs, but laments

We are always going to be low man on the totem pole and that won't ever change. And so, we are always going to have some parts of our department or university that probably are not going to listen to us and it's just a nature of it because we are at the bottom you know. 
Gina's colorful language doesn't convey an atmosphere of equality, collaboration, or acceptance. The tone during these parts of the interviews is one of acceptance of the situation. They are 'Just NTT' and that is just how it is. The NTTs seem to be reconciled to the reality of the situation. The label of 'Just NTT' has crept into the identity of some NTT faculty, who refer to themselves in this manner. The term appears self-deprecating. For example, Sandra a Specialist with a pedagogical degree and 15 years of part-time NTT teaching experience before teaching full-time NTT for 7 years, uses the term in passing when describing her employment at various universities saying she has only ever been 'Just NTT'. The terms 'only' or 'just' have become a part of the accepted vernacular and culture of the university, they are a part of the hidden transcript that says there is a division of TT faculty and then there is 'Just NTT'. Sandra goes on to explain that she is content in her job at the university and enjoys it so much she almost forgets her place. 'I almost sometimes don't feel like NTT. Even though that's in the back of my mind." She says she does not feel like an NTT faculty member, which begs the question, what does it mean to be an NTT? How should an NTT feel? And why is this always in the back of your mind? NTTs described the experience of being 'Just NTT' as not only having limited influence in decision making but also a lack of inclusion and appreciation as a contributing and valuable team member.

Despite sharing statements acknowledging the feeling they are 'Just NTT' three participants were emphatic that they were valued and belonged. These individuals felt they had a space, a voice, and a contribution that was desired in their departments. Vanessa, a part-time Aspiring Academic with a pedagogical degree, has taught in two departments on campus and shares the importance of having dedicated space, basic orientation, and supplies to aid in her inclusion. She commented, 
It's like, here's your desk and there's a stapler and pencils and tape. You know, like everything was there. They showed me around to the copy machine like they just, it was welcoming, and it felt like oh, okay, I have a job here. Not like I'm this outsider, that feels really awkward all the time. If that makes sense...You can actually be here in this room. Vanessa referenced the simple things one department did to help her feel like she belonged by providing a basic tour of the office and supplies. A stapler and a tour were enough to send messages to Vanessa of belonging and acceptance. Sandra also referenced having dedicated office space as a way of being included. She has taught for other universities finally feels accepted for the first time. She used terms like "important", "worthwhile" and "needed". When asked what made her feel this way compared to her previous employment, she referenced involvement in the department and acceptance by co-workers as well as an overall feeling of being valued. She discussed belonging, They involved me in the faculty governance. They give me an office. They expect me to have office hours...I feel like they are looking at me like 'Oh we really need you' above and beyond just an adjunct person that teaches just a course here and there. Uhm it's like I feel like I am looked at as a subject matter expert. I think through the inclusion in the things we are being included in as well as the way that we are treated by other faculty including our program director. I never had that experience before. It was kinda like I felt like it was kind of dismissive before. I don't feel dismissed at all.

As Sandra explains she is not just invited she is involved. Her feeling of involvement stemmed from having dedicated office space, being regarded as a professional by her colleagues due to her discipline expertise, and being involved in her department through committees and meetings. Involvement in faculty governance through departmental, college, or university level committees 
and meetings is one way faculty are connected to their campuses and become infused with the institution.

The NTTs in this study varied in their experiences with faculty governance. While the labor union contract states that NTT faculty are encouraged to attend departmental and organizational meetings, some NTTs felt they were either not invited or not included in these experiences. Inclusion or exclusion in faculty governance was another reinforcer in the identity of 'Just NTT'. Maya argued that NTTs were insignificant enough in the hierarchy that they were not even included in meeting invitations. She explained,

I would say some things you're not invited to if you're an NTT uhm sometimes I feel like the expectation is pretty low in terms of attending things... I feel like there's very little expectations and I know for a while we weren't even uhm NTTs included on some of the meeting invites. I think that's only uhm in like the last year or two that we've been invited to more. More meetings.

Maya discussed not being invited but also lamented that the expectations for NTTs are low. Contrast this with Sandra (above) that felt included because she was expected to behave as a professional by having content expertise and office hours. Other NTTs were invited but not required to attend which can also be problematic. As Sara pointed out, lack of attendance would mean she missed vital information to job success.

It's just weird things like that at faculty meetings. So, you don't have to go to faculty meetings, right? It's not required. But how are you supposed to know any information? (chuckles) or if I have felt like so if I don't go to faculty meetings but the minutes for the meetings aren't sent out until a month later. So, if I want to know anything that happened I would either be a month behind which then I would probably miss out on things I 
should've been doing and then why wouldn't you want to be involved in faculty meeting? (chuckles)

Sara laughed at the situation. Not being required to attend is an example of a public transcript that has messages of freedom and choice. However, the reality of missing vital information puts her and others in her department in a situation of interpreting the messages as 'you really should attend the meeting.' Unspoken expectations are hidden transcripts. NTTs in Sara's department agreed that there is an unspoken expectation of attendance. Tyler is a faculty developer that also teaches part-time as an NTT educator. He described the messages he received when attending a departmental faculty meeting as exclusionary. Tyler maintained,

I'm a little bit numb to it. But my first faculty meeting in my school, I felt like I didn't belong. And it's like not like an imposter syndrome, I felt like, oh, this is all the messages that you're giving are all for tenure track faculty. So even though I'm here and I'm an NTT and (specific person) is here and she's an academic advisor, this is really just tenure track information. And and [sic] then we're going to pass around a committee signed up, remember, tenure track, you're required to sign up for two of these, but everybody else, you know, sign up only if you're interested. I felt like oh, well, that's not for me also.

The tacit messages received by Tyler attempting to be involved during faculty governance were, you do not belong here, that's not for you, you are 'Just NTT'. These are messages of exclusion. These are hidden transcripts.

NTT faculty want to belong and be fully included. Vanessa shared her feelings of exclusion saying she felt lonely and disconnected using statements like "you're just like there" and "this lonely person who's just doing whatever ". Vanessa explained that she "just never felt like a part of the team." Vanessa recalled that she did not really connect with any of her 
colleagues. She went on to share a story from another NTT faculty member in her department that impacted her.

I think she's been here like 16 years or something. And she told me, it took me till 10 years before I felt comfortable. Like before, I felt like I had a job here. And I thought that's garbage. I mean, 10 years is a long time to get stability.

It was shocking to Vanessa that it could take 10 years to feel stable and accepted in the workplace. To be included as a part of the team, as a contributing member goes beyond an invitation to meetings. All three departments' administrators stated that NTTs were invited even Vanessa's. This finding suggests that perhaps administrators are not aware of the exclusion occurring at this level. The public transcript of being invited does not discount the hidden transcript of not feeling included. Latoya's insight helps navigate the variance in the results. She explained,

I want to kind of mention, it's kind of a delicate balance, because the reason why, you know, like non-tenure track, there are no outside committee obligation. And so, some people read it [as] feel very free from the point of view that they aren't asked to do these things, but that there are opportunities where you can volunteer if you choose to. Latoya's explanation may shed the most light explaining that NTT faculty are not required to attend meetings, which some find liberating and others view as exclusionary messages. This exclusion, however, continues to contribute to the identity of 'Just NTT'.

According to the labor union contract NTTs are encouraged to attend departmental and organizational meetings. Some departments have interpreted this as NTTs are not required to attend meetings. When the public and hidden transcripts provide conflicting messages, this can make it more difficult to navigate new environments. In higher education, there are distinct 
groups of employees with large differences in onboarding procedures and language that daily reinforces these differences in position type. The system is designed to privilege some over others contributing to the identity of 'Just NTT'.

The administrators in all three departments emphasized that NTTs are invited to participate as an option without a requirement. The NTTs in department B wanted to be included in more meetings and expressed a feeling of exclusion. Those in Department $\mathrm{C}$ felt they were invited but had no voice and NTTs in Department A felt an unspoken expectation that attendance and involvement are expected. This finding speaks to the fact that individual departments have their own set of cultural expectations regarding involvement that is not universal across campus and that must be learned navigating the public and hidden transcripts these will be discussed further in examining the role of NTTs.

\section{"I don't really see the categories"}

While administrators and NTTs both acknowledged a power dynamic and hierarchy present on campus, one group, that insisted they do not see differences in the categories of employees was faculty developers. That is not to say faculty developers do not believe the hierarchy exists, quite the contrary, the attempt to not differentiate between NTT and TT faculty comes from a seemingly well-meaning place of an attempt to minimize the impact of the division. One developer, Sue, explains

Well, I don't know that I've ever purposefully divided you know, the folks that I've worked with in terms of AP faculty, NTT, so I don't really see the categories as much when I'm engaging in that kind of work. I have always said that all the options and opportunity should be open to everyone, including graduate students. 
Faculty developers "don't see categories" to provide professional development opportunities for all employees regardless of tenure status. However, the researcher found that NTTs want their identity to be acknowledged by having separate professional development opportunities available to meet the unique needs of NTTs. This desire includes offerings at variant times of the day, days of the week as well as online to meet schedules for Freelancers and Specialists who may be working multiple jobs. Additionally, NTTs want professional development on topics relevant to their identities, such as how to teach developmentally to college students and juggling the NTT faculty lifestyle. Findings suggest that not acknowledging the differences present in NTT and TT appointments may be detrimental to participation in faculty development. NTTs may not realize they can participate in professional development offered by the faculty development center as it is not specifically marketed to NTTs. Gina was unaware she could attend internal professional development. She shared that she would like to attend professional development saying, ...or even feeling more, feeling more confident of attending some of the (faculty development) having more information about the (faculty development office) training that would have done an official like, we get emails, but I didn't know if I was allowed to go like if those were just for the tenure line or if that was for the non-tenure line. I didn't know what I was (chuckle). Until a lot later that I realized, oh, those are open to me too! Gina brings to light that NTTs often receive communication via email or through departmental meetings that is sent to all faculty but pertain only to the TT faculty. Thus, in the case of faculty development, findings suggest clarification should be made to explicitly include NTT faculty. By not seeing a difference in NTT and TT faculty, faculty developers may be missing opportunities to provide training for NTTs that may not be aware of the faculty developer's intent to be inclusive. 


\section{The Role of NTTs}

The researcher found differences in identity based on how the NTT faculty perceived their role. Role was defined by the researcher as the main purpose or function of the position. The way in which the NTT faculty members defined their role at the university was analyzed by department. This included the perspectives of both full and part-time NTT interviewed as well as opinions from administrators and faculty developers. During the study, it became clear that NTT faculty in each department had differences of opinions regarding the purpose of their positions. How the NTT faculty conceived the reason for their employment influenced their perceptions of the level of support, involvement, and expectations.

Those in Department A viewed the role of the NTT faculty as teaching from lived experience. NTT faculty in Department A focused on the fact that they had been practitioners in their specific disciplines prior to working at the university and thus carried content-specific job knowledge and practical work experience into their teaching practices. The NTT faculty in Department A felt important in their departments and that they had a voice as a contributing member of the team. All the NTT faculty in Department A were full-time and all but one had received the negotiated category of status. The NTTs described a departmental environment of collegiality where they could contribute to not only teaching but also to faculty governance, curricular decision making, and matters of importance in their department. They mention support from the strong network of other NTTs as well as TT colleagues that are willing to connect and engage collegially. The NTTs in Department A contributed to decision-making in matters of importance such as policy, procedure, and changes to the curriculum. However, they are very aware this type of inclusion is unique to their department and carry a level of guilt for their preferential treatment compared to their NTT colleagues in other departments. These NTTs are 
also aware this acceptance does not go beyond their department into the campus community. Sara had some strong words to describe this experience.

I don't know I feel. The department is good, the school is good, but I feel like if we were to bubble out into the dean's area that's where I feel like a peon.

Sara's term 'peon' demonstrates the lack of professionalism and prestige the position of NTT holds at the university. She goes on to explain it is no wonder committees at the university level have difficulty getting NTTs to serve. Indeed, why would you volunteer your time for no compensation, no impact on your evaluation, and be treated as a 'peon'? The NTTs in Department A described implied expectations outside the role of teaching, such as attendance at meetings and service on committees. None of these expectations are explicitly stated, however, all of the NTTs interviewed in this department are doing service and or research that is not required of their positions. Isabella explains the culture of expectation

I think it's hard with NTT's because it sometimes feels like we're not necessarily working for promotion so uhm I I [sic] know a ton of NTT's, at least the ones I've been around, do do [sic] things that aren't expected or aren't like yeah expected, but it does kind of still seem like well all the NTT's do it, so it is kind of expectation. Right? Like being part of the department is generally an expectation, but I think it's also enjoyable in my case. Like being part is I feel 100\% part of the department and I participate in department events and University events as if I am a full member of that of (specific department).

Isabella's voice echoes all the interviews from this department, they conveyed stories of feeling included as a part of the department but also feel they must do other uncompensated duties beyond teaching to be viewed by others as contributing to the team. These expectations have become a part of their role identity and a path towards acceptance. Administrator Pat agrees the 
NTT faculty bring a wealth of experiential knowledge and confirms the view that within Department A NTTs are viewed as valuable colleagues, however, within the system of the university there is a gulf between TT and NTT faculty.

Department B NTTs also felt the role was to teach and felt there was a strong connection to practical knowledge as several NTTs are practitioners in the discipline but emphasized their identity as filling in when TT faculty are not available to teach courses. This is consistent with the administrator's description of a department that offers many courses and sections and does not have enough TT faculty; thus, NTT faculty cover those courses. Department B faculty felt more tangential to TT faculty than their NTT faculty counterparts in Departments A or C. Three of the five participants used the term fill-in as a part of their identity.

The NTTs in this department mentioned collaboration with department leads/program coordinators, however, felt they had minimal input that could result in change, sharing they had experiential knowledge that is overlooked. Jake a part-time Specialist NTT that teaches for multiple institutions explains that he thinks NTT faculty could contribute to the conversation. uhm and it would make my time and my experiences feel valued. So, I mean the courses that I teach I kinda teach repeatedly. So, they are the same ones if maybe the lead of that course would reach out especially in their time for revisions to say hey do you have any feedback on this course even knowing that. And some of the adjuncts teaching that course are actually working in the field right now. I think we could provide a lot of uhm on the ground feedback in how students could maybe benefit more from the course so when they go back to redesign it, they can you know keep those pieces into account and I think that would help uhm in ensuring that the NTT fee valued and vested in what they are doing. 
Jake argues that being a practitioner gives him real-life experience that could aid the whole department in informed teaching. The administrator described attempts to invite and include the NTT faculty in the department, creating specific training and orientations to meet their needs. These trainings were valuable to the NTTs that attended them. However, being optional and at times not convenient for the NTTs, only one participant in the study attended. The administrator was protective of the NTT's time by not asking them to serve on committees. However, this was interpreted by the NTTs as being excluded. The administrator appeared unaware of the NTT's perception of exclusion. None of the NTTs in this department had obtained the stability of status and thus would be employed the shortest amount of time of the NTTs in this study. These NTTs also noted a lack of opportunity to become full-time status NTT employees but held out hope of this occurring. This group lacked information on the process to become status NTTs and this contributed to their identity of filling in for others, viewing themselves as more temporary workers with limited powers and input. This limitation coupled with uncertainty in employment will most likely mean these NTTs will not voice desires to the administrator.

The NTTs of Department C felt their role was also to teach, however, they emphasized that NTTs are hired to teach general education courses or courses that TT faculty did not want to teach. This is like Department B in the definition of NTT faculty being supplementary to TT faculty, however, Department C faculty had a sense of permanence with all but one having status and a strong awareness of their subordinate position. This department mentioned power dynamics and characterized their input as having no voice, especially when discussing issues with TT faculty. They felt supported by their chair but explained distinct social classes evident among the faculty. This group disclosed an additional tier beyond the typical TT and NTT faculty hierarchy. Participants described a layer below the NTT faculty on the power hierarchy 
of general education NTT faculty. This observation confirms having expertise knowledge is advantageous to position and power. Administrator Casey confirmed a power dynamic describing separate social events the NTTs had created for connection and belonging.

I recognize there is a power dynamic and while we do try to be inclusive and try to invite them in. I know some not all feel comfortable that thing and so it is, you know, it's all I think that's part of the reason why they sort of develop their own community that way. They remorsefully shared that the NTT faculty will never realize how valued they really are. Casey laments a system that doesn't embrace the NTT faculty. Casey explained there are leadership positions available in the department to NTT faculty to be included but at a smaller percentage of representation than their TT colleagues. Jason confirms that NTTs in Department $\mathrm{C}$ are present at meetings, but he said he felt their opinions are not as valued as the TT faculty. He commented,

It's been my experience that when NTTs and TT get together to decide something the TT, decide and the NTTs can either nod or fume. I hope it has changed. But basically, it's my opinion that the NTT's usually do not influence TT.

Jason epitomizes the feelings described by Department C NTTs that they are present but not truly included. The NTTs can agree with the TT faculty or sit in a meeting, present but not included. The identity of NTTs in Department C is one of teaching separately from their TT colleagues. 


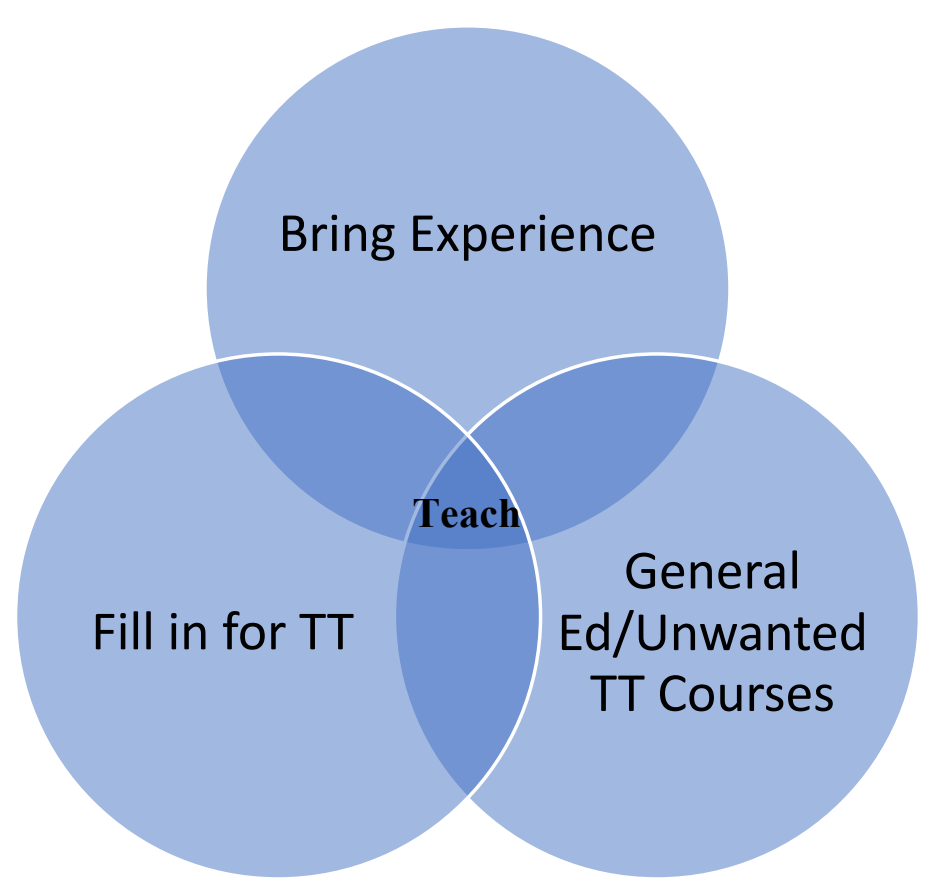

Figure 1 shows the perceived role of NTTs by department. Whereas all three departments agreed the role of the NTTs was to teach, there are differences in the reasons attributed for this teaching. Department A views their role as applying their experience from being practitioners. Department B felt they were to fill in for TT faculty. Finally, Department C faculty communicated teaching general education courses or those that the TT faculty did not have a desire to teach.

The environment in which NTT work has both social and political implications. As discussed by Bolman and Deal (2013) universities have clearly defined power structures regarding faculty governance, policy, and procedure. This was apparent during interviews with all research participants. These findings suggest that power structures present in a hierarchy establish systemic barriers to full inclusion and full acceptance into the professoriate. This is compounded by unclear expectations of work responsibilities, power dynamics, and feelings of value that are unique to each department's culture. This study found that the workplace for NTT holds many assumptions and tacit messages that impact the identity and role of being 'Just NTT'. 
NTTs that were alums of the institution in which they worked held an advantage in navigating sociopolitical and procedural expectations.

\section{Alumni: "Piece of Cake"}

NTTs that are alumni were more connected and acclimated to the university because of having past knowledge of resources both in policy and procedure. Ten of the NTT faculty participants were alumni of the institution in which the study took place. The subtheme of alumni emerged when the researcher noticed a pattern of a large number $(66 \%)$ of the NTTs mentioned not only acquiring a degree from the university but also feeling connected. Part of their personal identities at the university includes feeling a connection to the institution and to others who have also studied in the same space and cheered for the same team. The alumni NTTs had human connections as informal resources based on knowing colleagues who were past instructors of theirs when they were students. Additionally, alumni mentioned familiarity with the basics of institutional culture such as the universities mission and basic orienteering such as where to locate offices. This knowledge helped alumni NTTs to acclimate quickly to their roles. Their previous experiences as students contributed directly to their preparedness. When asked about acclimating to the university and the culture Marcus said he had no difficulties, "Piece of cake. Yeah, I went to school here so, so I feel very comfortable here." There is an advantage to having had years as a student to observe the institution. Those years bring comfort and understanding of the university expectations. Marcus was confident in his identity as an NTT in part because of his identity as an alumnus.

All be it students are most likely looking for different types of information than instructors, there would be some overlaps. For example, Isabella completed her graduate program in the department in which she now teaches. She began her NTT position with 
institutional and procedural knowledge including knowledge of Human Resources, university computer systems, course management systems, voicemail, campus mail, building locations, and campus departments for resources. Her words have an air of confidence as she lists the items that made the transition from her student role to her NTT role smooth,

I knew like a lot of those things. I knew how to use the phones and set up my voicemail and that kind of stuff ...yeah, I think because I went to school here because I want to graduate school here that made it super easy for me to get connected on campus. I am pretty involved. I am on like serve on of a bunch of different committees and like you know just volunteer and stuff across campus, but I think those are ties that I made during my grad work.

Isabella was able to start her NTT position with an understanding of her work environment. But she was also able to integrate and be included in committees and volunteering because she had a preexisting relationship and an insider's identity at the university. She is a model example of an NTT faculty member who was a graduate student and was then hired to teach one or two courses as a part-time NTT educator, eventually turning into full-time NTT faculty. This route to fulltime status is common for the NTTs in the study. Having been a graduate student at the university is a distinct advantage in knowing course management software, telephone systems, campus wayfinding, and connections to the campus culture. Contrast Isabella's onboarding experience with a participant that completed their degree work at an outside institution, such as Gretchen. Gretchen received information from the informal network of NTTs for procedural items and is looking towards context clues to understand cultural expectations.

So, for me, it has been trying to zone in and see what (the University) expects and wants of their faculty. What their goal is for their faculty more than anything. 
Gretchen is looking for cultural information. Maria also completed her graduate work elsewhere but attended the optional acculturation orientation provided by the university as well as a departmental level orientation designed specifically for NTTs onboarding. She stated that she felt more prepared than her peers as a direct result of attending these orientations.

Ifelt like that it really helped me understand. Helped me get the idea of the culture and all that. And if I didn't go to that I would've been lost and I have friends that chose not to go to that, and they seek me out for information that I learned at those orientations.

Maria demonstrates not only NTT teaching NTT as was discussed earlier but also the value of onboarding. The findings suggest that alumni acclimate and integrate into the university procedures and culture more easily than their non-alumni counterparts. This research does not suggest that only alum should be hired as NTTs, but that the acculturalization orientations and departmental level NTT specific onboarding may serve as an effective method in acclimating newly hired NTTs.

In addition to connecting with the culture and having knowledge of some procedures, alumni have the advantage of being known by some of their TT colleagues. Juanita acknowledged she is unique among the NTTs in her department in that her voice matters. She attributed this to her alumni status and connections as friends with TT colleagues. She shared that the connection is invaluable towards feeling accepted.

I do feel that I feel like I feel like I do have a voice and that it does matter in the most appropriate that's not true for everybody. And again, I'm in a unique position because sometimes there can be an understood line between the full PhDs and everyone else, especially NTTS. But I've never felt that way because I'm friends with those people, you know what I mean? They are. Yeah, they have relationships with them, but they're full 
professors, where I've been to their houses for dinners and things like that. So, I don't feel that ...but I think that interactions like that where you do feel like you're part of the community are invaluable, probably for an NTT.... A major part of it like I said is that I got my undergrad (university).

Juanita's opinion matters despite her NTT role and title because of her alumna identity. She is treated differently than the other NTTs. She described incidents where she had a voice and is professionally and socially included because they know her. Interestingly, developing a kinship and connection to the campus like the experience of being alumni occurred with those that attended the acculturation orientation. Another group of NTTs that hold more power are those with certifications.

\section{Essential Specialists}

At the beginning of the study, it was believed that holding a pedagogical degree or teaching in a specific discipline would factor into role identification for NTTs. This assumption was not supported by the data in this study. What did contribute to identity was NTTs with additional certifications or narrow training making them essential specialists. Dan, Gretchen, and Sandra were the three NTTS that felt their expert knowledge provided a level of job security. This went beyond discipline-specific knowledge because in all three cases these individuals also hold a certification or credential that was required to practice in their field. This additional training ensured they could fill a need of specialized knowledge others could not.

Feelings of contributing an essential role were emphasized with those NTTs that considered themselves Specialists as a part of their identity according to the Gappa and Leslie categories (1993) and having additional advanced training. Having been hired for content specific knowledge, and additional essential certifications this information elevated their role and 
provided a sense of value and security in knowing they had information to contribute to their positions as NTT faculty. Dan is a specialist with a content specific degree and a specialized certificate. He has taught in two departments as a part-time NTT faculty member at the University. He stated that he is called in as needed to teach and strives to be skilled in many specialized content areas so the university will call on him more often. He used a sports analogy and referred to his NTT faculty role as "bench" meaning a player that waits on the sidelines and is put in when needed for a specific task. Dan articulated his experience as follows,

It's a specialty type of career. And it's that's also a specific major of the (specific) department. So, they picked me to it was because of my experience, my work experience that they brought me in to do that.

Dan perceives his NTT Specialist identity as elevated with each additional certification he obtains. He discussed a desire to pursue additional specializations in hopes to be called off the 'bench' more often. Gretchen also shared the confidence that comes with having an essential skill set. Gretchen began as a part-time NTT educator and was hired full-time due to her expertise in her field. She identified as an Aspiring Academic, due to enrolling in a doctoral program, but otherwise felt she was a Specialist. She conveyed the role of NTTs as interacting with and teaching students. Gretchen stated she felt prepared to teach from past experiences as a graduate assistant and having taught as an adjunct at a different institution. Gretchen is in a unique position as an NTT faculty member since she has the highest degree possible in her field. She explained she feels valued for her expertise but acknowledges this is not the case with all NTTs.

Because I am (here Gretchen pauses to consider her statement before proceeding) as far as our profession. I am as equally qualified as anyone else, and I think that has always 
been a nice bargaining chip for me because I think our program directors also recognize

that. They understand that moving forward my position is essential in this program.

Gretchen perceives her skill set and qualifications as essential and this gives her a sense of identity in her specific discipline.

Not all who selected the Gappa and Leslie (1993) title Specialists identify as being essential. Another participant, Latoya, was unsure which category fit her as she teaches "only" general education courses, however, she decided Specialist was the best fit due to the content specific knowledge she holds. Her hesitancy stems from teaching general education courses and working alongside TT faculty that teach upper-division coursework. Latoya and other NTTs communicated that the general education courses were not viewed as important or valuable at the university. Latoya described the role of NTTs as teaching. She stated it is a valuable role and cost savings to the university as well as a contribution to allow TT faculty time to research and do service as well as teach major-specific courses. Her identity within her NTT position is entwined with her view of NTTs who teach 'just' general education courses, these courses do not require additional certifications like those needed by Gretchen. LaToya's shared identity serves as a stark contrast to the NTTs that consider themselves essential. Those that had essential credentials or expertise were uniquely positioned in a social standing of increased power within the two-party system. This expertise bolstered the NTTs value and increased their social standing. The identity of NTTs is also impacted by the presence of a union representing these positions on campus.

\section{Representation}

The NTT faculty are represented by a labor union. This subtheme includes mentions of the NTT labor union. Findings suggest that NTTs in this study are either unaware or 
unconcerned about being an active part of the labor union. The environment among the NTTs is apathetic toward the union. Mentions of the union in this study primarily occurred in relation to the term 'status'. Status is a negotiated longevity benefit providing some job security to NTTs. According to the union contract, once the NTT employee has worked eight consecutive semesters the employee receives a pay increase and has additional job security. Security comes in the form of consultation and education if the employee were to receive negative reviews, providing the employee opportunity to improve and retain their position. Essentially, there is an eight-semester probation period for NTTs in which they may be dismissed. This status language may be the reason some NTTs are not hired consistently in back-to-back semesters. Several NTTs interviewed were not aware of the term status, and several were unaware the union existed. Those full-time NTTs that had acquired status had little to say about the union, as was evidenced in a memo written by the researcher in April. At the time of the memo's creation, the researcher had contacted every member of the labor union board and received insufficient response to include quotes from union representative interviews. To help maintain confidentiality, memos and a document review of the labor contract and union meeting minutes were used as data to discuss the sociopolitical implications of the union. The text of the researcher's memo on the labor union follows.

The union really didn't come into play much. It was only mentioned in regard to stability or compliance with evaluations. Which is actually attributed to HR and not the union. NTTs appear to be aware that achieving status (so many semesters consecutive work stipulated by the labor agreement) provides constant contracts. However, the FT NTT don't seem to care. They either have status or view the union as unnecessary. Probably due to the lack of perceived benefits. 
Interestingly the PT NTT want the stability status would provide but seem to be very

unaware/uneducated about the union and what rights it affords.

The researcher found that being represented by the labor union was not an integral part of the NTT faculty identity, nor are the NTTs actively engaged in the union. The union representative described the NTTs as apathetic. They conveyed stories of active recruitment and little involvement bemoaning 455 NTTs on campus and only 87 pay dues with one person doing all the work for the union. While the researcher found the total number of NTTs (full and part-time combined) to be 628 and was unable to find the number that pays dues, the lack of involvement or awareness in the union is clear (University Factbook, 2019). Administrator interviews provided no new insights. NTT participants did not suggest that the union was part of their NTT identity rather the stability afforded through the negotiated right of status was. This right was viewed as more of a procedure from Human Resources than a negotiated benefit.

The identity of an NTT faculty member is as complex as the individuals themselves. Viewing the role of identity beyond the job description of teaching allowed the researcher to explore identity through the lived experience of the NTTs including department-specific sociopolitical implications. Understanding role identification can help to explain the NTT's perception of their experience. The term 'just NTT' demonstrates a subordinate classification in a two-party hierarchal system that favors TT over the NTT faculty. This statement has implications that minimize or devalue the position of NTTs as less than their TT colleagues. Other implications of role identity were discovered including insight into how the NTTs conceptualize the purpose of their positions and the impact of having been alumni of the institution in which they work. Those NTTs that had attended the institution also found favor by being known by their TT colleagues prior to becoming employees and by having some 
knowledge of university processes. The theme of identity was further explored by evaluating implications of Specialists being perceived as essential due to holding certifications and the role of the labor union in identity.

\section{Evaluations: What's the Point?}

Evaluations are typically used in a workplace to determine retention or continued employment as well as provide feedback for improvement. When asked to describe the departmental evaluation process NTT faculty and administrators explained two separate processes that are occurring. One process involves students providing feedback on their instructor for the semester and the other is that of administrators' evaluation of the NTT faculty, typically performed annually unless the employee has status, and then it is conducted at the negotiated rate of every three years. The administrator's feedback was described by the NTTs as perfunctory and characterized as required. Indeed, it is a required process that is implemented by Human Resources and described in the labor union agreement. Thirteen (87\%) of the NTTs interviewed felt the evaluation process lacked substance to aid them in growing in their trade. However, all the NTTs used the student feedback received to inform their teaching. When asked how evaluations are used to determine retention NTTs were either unsure, adamant that they were not used, or dismayed that they had never themselves considered this question. Many of the participants described evaluations as not relevant or did not receive feedback that would assist them in their teaching. They do want more robust evaluations from administrators but do not feel they can request it due to the precarious nature of their positions. The process, motivations, usage, and intent behind NTTs evaluations resulted in the theme of evaluations: what's the point? This theme includes the subthemes of evaluation: process transcends purpose, retention: I 
really don't know, self-development and growth: coaching for improvement, professional development both a means and an end, and student feedback. The point of evaluation is unclear for this vulnerable group. The discussion for this theme will begin by looking at the negotiated process of evaluating NTTs as managed by human resources.

\section{Evaluation: Process Transcends Purpose}

The labor union agreement clearly details an evaluation process that was negotiated between the labor union and the management of the university. Article XVI, Faculty Evaluation Process of the labor union agreement explains the purpose and process (Agreement, 2017). The purpose is two-fold, information for employment decisions and facilitation of self-development and growth. The contract stipulates that both full and part-time NTT probational faculty are to receive annual evaluations. Probation for NTTs is eight consecutive semesters at which time the NTT faculty member would be considered status and receive evaluations at least every third

year. Materials from both spring and fall semesters are to be collected. Written procedures are to be in place for evaluations and the union contract provides general guidelines for materials to be used in the evaluation. Guidelines include having NTTs provide a self-assessment as well as student evaluations and informing the NTT faculty member who will be completing the evaluation if someone other than the chair. The three departments studied for this research project; all followed this process. The union contract outlines that the NTT faculty should be evaluated specific to their primary duties of teaching and thus evaluated based on quality of instructional performance. The artifact encourages six main areas of evaluation of instruction. The evaluation of instruction will consider, but not be limited to, execution of assigned responsibilities; command of the subject matter or discipline; oral English proficiency as mandated by (state) statute; ability to organize, analyze, and present knowledge or 
material; ability to encourage and interest students in the learning process; and in student advisement and direction given for student activities (Agreement, 2017). Self-assessment and student feedback are to be considered as a portion of the evaluation. However, the artifact reminds the reader that student evaluations are only one piece of information and that subject matter and nature of the course also hold importance. Procedurally the NTT faculty is to receive a document informing them of the results of their evaluations. If the resulting evaluation is less than satisfactory, the artifact outlines a coaching procedure in which the chair meets with the NTT faculty member to "clarify and establish expectations and goals for the employee's future work." This information is to be put in writing.

All three administrators explained evaluation processes in their departments very similar to the ones described in the labor union agreement. While the chair of Department A sometimes does evaluations themselves, this has also been delegated to the program coordinators/leads at times. In Departments B and C, the Departmental Faculty Status Committee (DFSC) charged with TT faculty appointment, salary, promotion, and tenure assists with NTT faculty evaluations. The committee is comprised of TT faculty only. Departments B and C provided the researcher documents for review including a template letter that follows the union contract nearly verbatim. Department C provides their NTTs an outline from Human Resources on items to include in a self-assessment. This includes items such as primary duties, English proficiencies, and contributions to the department, it too closely follows the labor agreement.

Department B provided a template email that is used to schedule classroom observations. This email includes a request for a pre-observation meeting. Department B was the only department of the three that met with NTTs prior to observations to discuss the goals of the observation. This department provided a pre-observation form that the evaluator can use to 
determine the NTT's teaching philosophy and professional goals. Department B also provided evaluation forms to guide the observer, one for online courses and a separate document to be used for courses with face-to-face delivery. The evaluation form provided a detailed explanation in each of the four areas to be evaluated that again mirrors the union contractual language. For example, expertise includes command of subject matter as well as the use of real-life anecdotes. This information is rated on a Likert scale ranging from strongly evident to not in evidence. While the forms do not address student advisement, they do address all the contractual evaluation areas regarding teaching.

All three departments had an appointment, salary, promotion, and tenure policy document for TT employees. Department A was the only department that has one such document specific to its NTT faculty. This is a unique document that appears to have been voted on by the NTT faculty implying some level of faculty governance and inclusion of the NTTs in decision making. This document is evidence that the NTTs in Department A have a voice at least at the departmental level and is consistent with interview findings and the differences in departments discussed earlier. The document details the evaluation process of self-assessment into what this department terms a dossier. The dossier includes a curriculum vitae, the establishment of goals and evidence of accomplishment in teaching, and if applicable the areas of service, professional development, awards, and scholarship. Clearly delineated descriptors are identified to evaluate the performance of teaching on a five-level rating scale. The document does contain the caveat that it is not to be used as a checklist stating ...high standards of professionalism and collegiality are prerequisite to all facets of successful faculty performance. 
Department A has high expectations for its NTTs but also tries to include them as colleagues according to this document. This could be why the NTTs in Department A said they feel they have a voice in decision making, but also an unspoken departmental culture of needing to do more than teach. These NTTs felt there are unspoken expectations that require additional service through things such as committees and faculty governance beyond their contractual role of teaching. While these NTTs do not necessarily want to be paid for these tasks, they do want recognition and evaluations that acknowledge this contribution. Sandra and four others in her department discussed expectations of their positions that go beyond teaching. These expectations were not explicitly discussed by their supervisors. However, each person has learned that being involved in committees and projects is strongly encouraged. Sandra explained the role of NTTs is to teach but that she has come to realize NTTs are expected to do more. ... I felt pressured to at least just be involved more than just being a teacher...Because that's the type of feedback I'm getting in the (School) director meeting that that person mentions uhm the things that I have done that are above and beyond that they really really [sic] appreciate. So, I think maybe, now that I think about it and I'm talking about this, I think it's possible that I do some of those things because I don't want my teaching evaluations to be the only thing, I'm evaluated on..... I have come to realize that uhm we are being we are I don't know that I would say expected, but we are asked to do much more than just teach. Such as serve on committees a special project that may be or may not be related to our course the courses that we teach.

While Sandra did not use the word expected she conveyed a sense of requirement and pressure that other NTTs in Department A shared, being asked to do more than teach and having this information be included in their evaluations. Sandra and the others were hesitant to disclose this 
information. But, as Sandra demonstrates, she was learning the expectations of her position by observing and comparing with other NTT faculty in the informal network. As service is also included in Department A's evaluation documentation, praised when it occurs, and openly discussed how colleagues contribute to the department, the implication for NTTs in this department is that it is expected.

Observing teaching is not explicitly stated in the union agreement. The agreement mentions only student evaluations and NTTs self- assessment. Teaching duties are to be evaluated based on a command of subject matter, ability to organize and present material, ability to interest students in the learning process, and student advisement. Additionally, there is a communication stipulation regarding having command of the English language. Departments are using observations as a source of information in addition to the NTT faculty self-assessment and student feedback.

Some of the NTTs interviewed received observations to evaluate their teaching, others had not. Those that had been observed found it to be on an unpredictable schedule with having observations some years and other years not receiving an observation. Others had disjointed experiences, for example, Jake had an observation but never received feedback from the observation. Since observations as an evaluative method are used in some departments and not others, this can be confusing as NTTs share information through the NTT network. NTTs are assuming their teaching should be observed every semester and those that are not described this omission as another area in which they are overlooked. For example, Maya teaches in two departments at the university and has been observed in one department every academic year and has never received an observation in the other department. She concludes one department is doing evaluations properly and the other is not. 
As the research dove into the practical applications of the formal evaluation it was discovered that observations were offered inconsistently and the evaluation process outlined in the union contract was followed regarding the process, but not regarding the described purpose. The purpose of the evaluation is to make employment decisions for continued teaching assignments and to aid the NTTs in self-development and growth. All three departments have documentation on compliance but no examples of feedback or growth for the NTTs' pedagogical development. Since evaluations are compliance-driven summative instruments, NTTs are unsure how these are used to determine retention, this will be discussed next.

\section{Retention: "I Really Don't Know"}

Nine of the NTTs interviewed (60\%) did not know how their evaluations were used to determine retention. Five (33\%) felt evaluations were not used to determine retention or rehire and only one person (6\%), Latoya, said positive evaluations resulted in obtaining status. Those that did not know how their evaluations were used responded to the researcher with uncomfortable concern. Some laughed nervously, others looked away or shuffled in their seats. The NTTs had assumed evaluations were done for some reason but they did not see a connection to course assignments as a direct result of evaluations. Vanessa is a perfect example of the realization that occurred during the interview. She began with a basic statement, "I don't really know how it (here she pauses to think) I mean, I think that they look at it (another pause as her words trail off)" then as the conversation progressed, she tried to connect her evaluation experience with the courses she has been assigned to teach. In this quote, the reader can hear Vanessa walking through what her experience with evaluations has been,

I really don't know. Because I feel like my evaluations have always been great. And I go from this overload of classes, which must, which makes me think like, I am doing a good 
job, and then to like barely any. So, I don't, I don't even think they're using it in that way. If that's what we're ending up with. Mm-hmm (she trails off thinking)

Vanessa reasoned her positive evaluations are the cause of her being assigned an overload of classes. However, she could not reconcile the fact that she also has had semesters in which she teaches only one course. This incongruence was confusing to the NTTs interviewed that assumed doing well on evaluations should result in being assigned more courses. Traditionally in workplaces evaluations are directly linked to continued rewards, recognition, or continued employment. However, on this campus course assignments are determined in an organized sequence dictated by the union contract. The selection of course faculty begins with TT faculty then progresses to NTT faculty with status and finally to NTTs without status. The confusion for Vanessa and NTTs like her comes from a lack of clear expectations and information on evaluations. These NTTs are gathering information on evaluations from the NTT network rather than a formal orientation, mentoring, or evaluation process. Many of the NTTs interviewed were not aware of the language of the labor union contract, the existence of the labor union, or how their evaluations are used to determine retention. Gina is an example of NTTs that are unsure how evaluations impact retention, she explains

Uhm ok my my [sic] answer is I don't know that they really are. I haven't seen that they negatively impact anybody's employment. So, I don't know. I really don't.

The NTTs were uncomfortable with the fact that they do not know what would or would not impact their retention. As Gina reflected, she has seen coworkers receive negative evaluations and continue to teach. Grappling with the fact that this information is unknown is disconcerting and participants even asked the researcher for answers, another evidence that NTTs are seeking 
information from other NTTs that may have gleaned this knowledge. Jada reflected on her own evaluation experience

To me, it seems like it's kind of a big waste of time. What is the meaning behind it because nobody even bothered to follow up? If you spent all this time putting all the evidence together writing all the stuff and nobody even remembered it was like I think it just had to go to HR and stuff and be documented. No one has ever followed up with me on any of that (chuckles) it's been quite some time since my last formal evaluation so (her words trail off).

Jada called the evaluation process a waste of time and felt the department is going through the motions out of compliance with HR. Since no one had discussed the results of her evaluation, it lacked purpose specific to professional development and growth and is being completed as a requirement. NTT's view this as more evidence that they are undervalued.

NTTs that met with administrators to discuss their evaluation results discussed that they lacked substance. Departmental evaluations were described as a formality that is not helpful. Sara said she hasn't received feedback that would aid her in her teaching practice. Her experience follows,

Uhm from the director standpoint I feel like I've never really gotten any feedback of changes you know. I've always just gotten, keep doing what you're doing.

Sara describes her experience as being stuck in a rut. She had a response like many NTTs across departments if they do receive evaluations the content is basic information reflecting the requirement of the evaluation only and did not serve to provide direction towards areas of improvement, specific pedagogical enhancements, or professional development. Dan sarcastically said he just puts his evaluation in his scrapbook as a reminder of how well the year 
went. He explained the form letter he receives typically just affirms the information he provided from his self-evaluation. Jason described an annual routine of updating the numbers and submitting essentially the same materials year after year. In short, administrative evaluations are not being used by NTTs to inform teaching because they typically are not receiving information that would aid in this process of professional development or retention.

Administrators shared their perspectives on the NTTs evaluation process sharing trepidation that the process is not helpful and relying on personal interactions to determine retention. Pat disclosed

I don't know if I did it right and I don't know if I did it well... you know you look at all those materials and try to (chuckles) summarize somehow you know what what's the person does and what you you [sic] know what your sense of of [sic] their strengths and maybe weaknesses might be and communicate that to them in a letter.

Pat's laughter shows that they realize the task of summarizing and affirming what a person does in one letter is daunting. This task is complex and compounded by having many NTTs to evaluate every year. Casey has a large number of NTTs in their department and says many have already reached the negotiated state of status. As a result, the evaluations are not really used to determine retention as their positions just 'roll over'. As was explained earlier, once NTTs receive status their positions are more secure. If classes are needed to be taught, the NTTs will receive contracts the following year and are then only be evaluated every three years. Alex also does not rely on evaluations to determine retention. They share this perspective, I would say, in addition to day to day, kind of my day to day feeling about how the NTTs are doing and, you know, student responses that come directly to me that evaluation form 
I shouldn't say the form that evaluation process is what strongly determines whether we would retain an NTT or not.

Alex does not rely on the evaluation to determine retention, but a sense of their knowledge of how the NTT faculty member is performing. The NTTs that feel the evaluations are not used to determine retention, appear to be correct.

Departments B and C both use the department's DFSC committee to aid in completing evaluations as it is a time-consuming task. For consistency, these departments have form letter templates that are used to communicate summary results to the NTTs. While using a standard format is helpful to manage the task it does fall short in providing individualized substantive and formative feedback. This form letter is an artifact from the departments that is value-laden to NTTs. The NTTs explained the letter is typically a summary of their self-feedback and information from the student evaluations they already have reviewed. Thus, the process is viewed as perfunctory and sends the message that the NTTs are not worth investing time in to provide guidance to develop their craft. This artifact reinforces messages that NTT are 'Just NTT' and not valuable teaching professionals. Other NTTs explained that the evaluator doesn't know enough about the course content or pedagogical practice to provide useful feedback. These NTTs communicated frustration in being evaluated by someone who is not specialized in teaching. Maya shared her perspective on administrator evaluations

He doesn't know anything about (specific topic) so it's always very positive ...uhm but he can't really help me in that regard, does that makes sense?

Maya's perception was that her evaluator was not skilled in being able to provide information to aid her growth and professional development and therefore the evaluation was cursory. Overall, NTTs in all three departments felt their evaluations were not used to determine retention nor 
were they helpful at providing information to grow in their teaching practice either from lack of substance or expertise. Administrator evaluations were perceived as perfunctory. Only two NTT participants had positive things to share about the administrative evaluation experience they will be discussed next.

\section{Development and Growth: Coaching for Improvement}

Two administrators and two NTT faculty mentioned a coaching model of evaluation in which constructive criticism is conveyed with an opportunity to improve and then reassess at a late date. The goal of the evaluation is to make the NTTs aware of specific areas that could be improved. Alex explains they use this procedure for NTTs that have received negative evaluations.

What I will do is, meet with them, talk with them talk about opportunities for growth, and then watch the following semester, see if those have been addressed. You know, if there is a pattern of evaluations that don't lead us to believe that they are providing a solid student experience, we wouldn't bring them back. But I would say that for the vast majority of those kinds of concerns they are addressed with some coaching and then the NTT making a solid effort to grow professionally.

Alex discusses remediation and coaching for NTTs with negative evaluations. Developing a remediation plan is helpful for employees that have received negative evaluations. However, this coaching model was not described for NTTs with positive or neutral evaluation results. Clearly communicated detailed feedback could assist NTT's that have positive evaluations in growing professionally as well as those developing after an unfavorable evaluation. Maria was one of two NTTs that had a coaching experience connected with positive evaluations. She described how she used her evaluation results to select professional development. 
Yes, I was able to use that ...uhm I was able to kind of zone in on me specifically. I looked at OK what do I know that I need to work on one if I've been told that I need to work on and then I really use that over reflecting on my practices. I did my own research my own readings and then I went to some of the workshops that focused on that and then I got evaluated the second time. I kept a written dialogue of kind of like OK how am I going to quote-unquote prove that I can try to improve my practice and then the next year when I did that evaluation, I was able to say OK these are the things I've done to learn from my past evaluation of that make sure to grow from my past evaluation.

Maria was able to incorporate feedback into an action plan including pursuing further academic readings and professional development to grow her teaching practice. Specific pedagogically sound feedback helps NTT faculty identify areas that need improvement. Observations with detailed feedback were also described by Vanessa.

I know, especially my second one, the lady that observed me gave me more tips like maybe you should ask questions in this way, or, you know, have them watched the video before class rather than in class. Just things like that. Which is really helpful to get another person's thoughts.

Vanessa's evaluator was able to note observations from Vanessa's teaching style and provide specific modifications that could be easily implemented such as class sequence and reflective prompts. Vanessa was appreciative, applied these changes to her practice, and saw improved evaluations as a result. This feedback loop was perceived as supportive and valuable not only for individual professional growth but also to elicit connection to her academic department. With only two participants sharing that they received detailed feedback, this practice was more the exception than the norm for the participants in this study. Without direct substantive feedback 
that is directly related to self-development and growth, or employment decisions NTTs use the NTT network to answer their questions of how to receive future employment contracts, how to develop their teaching and how to become a valued contributing member of their department and the university. One of the theories that exist in the NTT world for ensuring continued employment is to make yourself valuable by contributing to the department as is the case with the NTTs in Department A view of expected service discussed earlier. Another method to demonstrate competency and possibly gain favor and thus continued employment is through professional development.

\section{Professional Development: Both A Means and An End}

In this study, the term professional development was defined to encompass both faculty development specific to pedagogy as well as a more holistic offering of resources to expand a faculty member's skill needed in higher education. While Human Resources, departments, colleges, and external agencies all provide professional development opportunities, the term was commonly equated to those services provided by the on-campus faculty development center. Professional development was conceptualized by study participants as both a means to an end and an end itself. Meaning that while professional development was viewed as helpful for growth it was also connected with the perception of necessary for positive evaluations that would ultimately impact NTTs having contracts renewed for continued employment. Therefore, some NTTs certainly participated in professional development to learn, but others were motivated by the potential of job security.

Both full and part-time NTTs felt that faculty development centers helped NTTs to stay current in technological trends such as the course management software. However, NTTs also acknowledge professional development as helpful for items such as learning new methods of 
classroom instruction, incorporation of ideas on globalization or diversity, and quality instruction. Latoya summarized professional development as teaching and technology. While professional development is viewed as beneficial to improving teaching practice, participants mentioned difficulty in accessing programs at times that were convenient for them to attend. This is not surprising considering NTTs often hold jobs outside of campus or teach for multiple departments and thus have schedules that may conflict with offering times of professional development training. Jake suggested that not only a variance in the timing on offerings but also who provides the development sessions vary to increase the NTTs connection to campus professional development. The faculty developers were also aware of this limitation and are moving towards more online offerings of workshops for the greatest flexibility. Interviews for this study took place during the summer of the Covid-19 pandemic and many of the professional development sessions had recently been moved to a fully online environment. Faculty developers shared the plan was to move these trainings to an online environment and the pandemic merely sped up the timeline for this goal. Both NTT faculty and faculty developers agree that this shift will allow flexibility in workshops and increase the possibility of NTTs' attendance.

Professional development is viewed by NTTs as both a means to an end and an end in itself. Maya's words demonstrate her desire to grow as an educator, but also her awareness that professional development may impact evaluations. This is underscored by her anticipation that she will receive stability in her position by being assigned more courses.

I want to grow but I also know that like the better teacher I am the better evaluation I'll get and then hopefully the more classes I'll be assigned.

Maya is not unlike her peers in their view that professional development can serve multiple goals. Maria agreed, explaining that she uses her attendance at professional development 
sessions to inform her teaching and bolster her evaluations explaining that if she had a negative statement from a student evaluation, she could show that she tried to change by attending a workshop on the skill to address the concern. Sara however doesn't feel that attending professional development provides enough of a direct impact on her evaluation to make it worth her time.

uhm but I feel like almost coming from the directors it was kind of like a why bother like this is great but you know we can't give you anything except a pat on the back so if you're going to do this, you're going to do it for you which is great I mean that's something you have to go in knowing... Right, when I had many directors say all I can give you is the pat on the back so if you don't need any more pat on the backs then why are you continuing to do more?

Sara explains the difficulty facing NTTs, attending professional development only serves to improve your own skills, it has no direct link in the current university system to promotion or retention.

It is of note that Department B offers professional development workshops specifically for their NTT faculty. This is a newer program and was mentioned by the administrator in that department, but only discussed by one participant in the study. This same department offers an NTT specific orientation in the fall semester. Again, this is a newer concept and attendance is voluntary, but they are the only department of the three to offer these types of programs. Vanessa attended the new workshop.

So last year, they had they offered for the first time some NTT PD for our department. I wouldn't say it was super helpful. But I know that they're trying, right. Like they're trying to put something together, which is better than the first two years. So, I appreciate that I 
did it. Because again, like, I want to show that I want to be here. And I want to be doing this and please give me classes, because I need a job.

Vanessa acknowledged that this is a new venture, but also demonstrated that she attended not only for the professional development but also to demonstrate her efforts in wanting to have a more permanent position in the department. Another motivation for professional development mentioned was to learn the campus culture and to have a sense of connection or community. NTTs were looking for opportunities to connect with colleagues.

NTTs do attend professional development for its own sake centered on training that pertained to technology and growth in pedagogy. Sandra shared emphatically that the faculty development center was the largest help to her acclimating to her role as an NTT.

I think the greatest help (word emphasized with increased volume and slow annunciation of the word greatest) with trying to improve my teaching has come from the (Faculty Development Center).

Sandra uses faculty development to expand on skills stating that it was a long time ago when she first learned to teach. The NTTs that attended professional development for its own sake were selective in the sessions they chose, looking for topics that would help them to grow professionally. Again, scheduling was mentioned as an issue with NTTs remarking the desired trainings were not available at times they could attend.

The study suggests that the role of faculty development from the perspective of the NTTs is to aid specifically with technology and pedagogy. This finding affirms the espoused belief from both full and part-time NTTs that the function of NTT faculty is to teach. The connection is to grow in a profession focused on the role of teaching, the development would be in the arena of pedagogy. Technology is seen as a method to enhance pedagogy, for example using the 
university course management system grade book. All fifteen NTT faculty participants reiterated that teaching was the primary purpose of their jobs and thus a finding that professional development is intended to bolster teaching and technology skills aligns well with that theme. Additionally, the NTT faculty are aware that despite being hired to teach, attending professional development can be used to bolster their evaluations. The most important aspect of evaluation came from student feedback which will be discussed as the last portion of the NTT evaluation experience.

\section{Student feedback: I Always Read It All}

Students are provided an opportunity to give anonymous feedback on each course at the end of the semester. The NTTs all agreed that they reviewed information from student evaluations every semester. NTTs most value the comments students write and typically look for consistent patterns that reflect areas for improvement. The consensus is that NTTs make changes in their pedagogical practice as a direct result of the student feedback. Dan explained there is both a quantitative and qualitative portion of the student evaluation. Numerically, the NTTs are given information from a standard set of questions to see how they compare in performance to faculty (both TT and NTT) teaching similar courses. Qualitative information is freeform and allows students to share any suggestion on the course or instructor. More insights from Dan follows

I read every comment. And I use it to make a little chart of suggestions from especially in the early going. Again, I could tell you tales, but I've, I've significantly revamped my teaching style, what I do in the classroom as a direct result of what students have commented on in those surveys. 
Dan not only reads all the comments but also takes the time to put them into charts, reflect and modify his practice all from student feedback. The feedback may also be used by the NTTs to confirm what they perceive as working well pedagogically within the course. NTTs felt the students were a more reliable judge of the course as they are aware of the entire semester rather than a one-time observation. All three administrators referenced student evaluations as a contributing factor when formulating the administrative evaluation. The student statements matter. Both NTTs and Administrators rely heavily on student feedback as a source of data for evaluations. This information aids in pinpointing areas of improvement and future professional development. However, NTTs agree that at times student feedback may be arbitrary or a reflection of personal likes/dislikes of the student rather than a reflection on their teaching practice. These types of comments are typically reviewed and ignored, with NTTs saying they try not to take it personally. Gretchen discussed self-reflection

Uhm if I do see any type of common thread in a class, of course, there is always the comments where you get polar opposite comments. I tend to take those with a grain of salt. But if I see sort of a common thread uhm I'll start using those as a way that I can potentially change pieces of the course from year to year. And then as far as my actual evaluations from my peers for teaching, program director, or school director, I don't tend to get that much feedback. Gretchen explained that she will sometimes receive contradictory feedback from students that she reviews with healthy skepticism, however, if there are patterns, she will make changes. Gretchen also said that compared to her administrator feedback, the students provide more substance. This was a common reflection from the NTTs who read all the comments from all their students and consider the criticism in context. 
Evaluations aid employees by providing feedback relevant to position expectations and performance. They are tools that administrators can use to inform their decisions on retention and encourage professional development. This study found that NTTs are lacking in substantive feedback from administrators but are using the feedback from their student evaluations to inform their teaching. NTTs desire more robust evaluations. This and other desired supports will be explored in the next theme.

\section{Value: It's Not All About Money}

Most jobs have aspects that could be improved or areas in which the employees want changes to be made. What is unique about NTTs is their vulnerability paired with often unclear expectations, limited support, and distinct power hierarchy in higher education. This combination creates an environment where NTTs are learning from the NTT network the expectations and evaluation criteria and feel powerless to request changes for fear of losing future employment opportunities. The NTTs interviewed wanted support beyond pedagogy and beyond pay increases resulting in the theme of value, it's not all about money.

References to being underpaid or wanting to increase pay were not absent in interviews with administrators, union representatives, and NTTs alike. While pay rates were not a focus of this study, they were mentioned infrequently as a needed item. Conversely, the union contract has pay rates as the main emphasis of the document. The mentions of pay equity in the interviews with administrators and NTTs were directly connected with references to the amount of work NTTs are providing the institution. This is especially the case in incidents where NTTs felt they are being asked to perform tasks beyond the scope of teaching. For example, Maya explained her desire to continue doing research and felt this brings value but since this is not a part of NTT work, she is not benefitting from this contribution financially. She mentioned having 
higher expectations for NTTs as a positive, but also included the fact that employees should be paid for doing additional work. Sara agreed and voiced frustration that service and committee work is uncompensated. While NTTs seem to understand the pay rate is out of their control, they also internalize this and the lack of recognition and job stability as a sign that they are not important or valued.

The concept of being valued includes holding someone in importance. This theme emerged early in the study and includes support received or desired by NTTs as a measure of their worth or value to the university. The perception held by the NTTS is the university will spend time and resources on the assets that are of value. Therefore, if NTTs are valued, they will receive material supports and the university will invest in them as a group of important individuals to the campus community. Value is evidenced through providing orientations, resources, professional development, mentors, effective feedback, and opportunities for inputs. Very few of these things are currently occurring. The converse is true, when NTTs are excluded from meetings, emails, professional development or professional supports the message received is that they are not valuable to the university. Vanessa was asked what could be done overall to support her work, she concluded, "like just having some internal feeling of value in the department." This was revealed after she mentioned the importance of job stability. She would feel more valued by her department if her position was more stable. Administrator Alex confirms the idea that NTTs may not realize their value to the department saying, "Not sure they always recognize how valued they are." The original interview question "what could be done to better support your work as a teacher?" produced responses that had little direct link to teaching, the main function NTTs identified for their positions. Rather, what was repeatedly discussed were items that would aid NTTs in feeling valued. Using Grounded Theory, the interview 
question was modified slightly to inquire, "what do you think could be done better to support your work as an NTT?” This language change still encompassed teaching but encouraged participants to discuss other aspects of the experience of being NTT faculty. The resulting answers from interviews produced an in-depth picture of the experience of being an NTT faculty member and what changes could be made to be more supportive of this group, resoundingly the answer was value.

Feelings of value can come from multiple sources that were grouped into subthemes; inclusion, stability, recognition and supports in performing the requirements of the job. NTTs often spoke of wanting to be seen as a contributing team member, a desire to be valued, or currently experiencing a feeling of undervalued and wanting recognition. Additionally, stability in both having consistent contracts and in which courses are assigned was desired as a sign of value. The last area entitled 'better at my job' encompasses supports that directly relate to improvements in the role of an NTT faculty member's responsibilities. This included references to professional development, funding for continuing education, onboarding processes, mentorship, check ins, and evaluations. These supports focused on support for the job overall or the experience of being an NTT. The desired supports to aid in a sense of value start with a strong need to belong.

\section{Inclusion: "I Just Never Felt Like a Part of The Team"}

Inclusion was defined as a desire to be included in decision-making or departmental happenings, to feel like a part of the team, or have representation. The researcher found that NTTs have a desire to contribute to the work in their departments not only by being on committees and attending departmental meetings but also by having a say in what occurs within the department regarding decisions that impact the courses they teach. NTTs in Department A 
felt connected and valued by their departments in part by having a voice in departmental and curricular matters. Those in Department A, however, are aware this is not the case for their counterparts across campus. The NTT faculty in Departments B and C want to have a voice. These faculty speak of wanting to have representation on curricular committees and being able to contribute to the conversation in areas in which they have direct experience as practitioners. NTTs also emphasize that contributing to the curriculum would help in feeling valuable to the team. The desire for a voice goes beyond attendance at a meeting. Gina explained that not only should there be more leadership opportunities for NTTs but also an environment of inclusion should be created for NTTs to feel safe in using their voices.

And I think that that needs to, there needs to be something that changes to allow everybody to have a voice without fearing there would be some kind of repercussion, to say, hey, this isn't as fair across the board as what you think it might be.

Gina emphasizes the reality that NTTs are a vulnerable group, aware of their low place in a hierarchical system, and that a desired change would be the ability to contribute without fear of negative ramifications such as loss of work. Having a voice is not just about being included and involved but also about being a contributing member of the group. Contribution is a component of inclusion among colleagues. Administrator Pat agreed that a desired support for NTTs is to be included. They state,

Uhm and I think that the kind of support I don't know if this makes sense but the kind of support that I have heard from NTTs in my role this past year has been more of you know wanting to be included and appreciated being looped in on different opportunities are.

Pat phrases inclusion as being looped in, having information about activities in the department, and belonging. They also mentioned appreciation which is discussed later as a finding termed 
recognition. This administrator is aware that NTTs want to be a part of the group. Vanessa used the word team and this term sums up what was meant when NTTs spoke of wanting to be respected, accepted, and included.

But just having from the beginning of when NTT start, you know, giving them those resources and letting them know like, hey, you are part of this department. You're You know, you're welcome to come to these things and we want your input. I just never felt like a part of the team.

Vanessa pointed out that what would make her feel like a part of the team is being welcomed and being asked to contribute. Team is the term used by NTTs in Department A when they spoke of being included by colleagues. Teams work together for a common goal with each individual being an integral but unique part. NTTs want to be included as a part of the team in the department and the college as well as across campus. Sara explained that while she is included in her department, she does not experience that same inclusion when she has tried to be involved at the college level. Latoya added that decisions are often made that impact the courses she teaches without her input, simply because she is an NTT as opposed to TT faculty. Maya found the lack of input alarming,

uhm, I think the way that some of the tenure-track treat us is like a huge, huge issue kind of elitist hierarchy within the university uhm having been there now for a while is a little bit alarming. Uhm, you know the pay is not great but I think a lot because we're not expected to do service or research, I think it would be nice if that would be included and I know that once you start including the things, you're like knocking on a tenure-tenure track uhm door but just like uhm to see uhm value in what we're doing. 
Maya wants to be included but also treated well for her contribution. She again uses the term valued. Value is more important to her than a high salary, just to be a part of the team and to be valued for her contribution. Value was discussed often in the NTT interviews in connection with inclusion, but also with stability.

\section{Stability: "I Don't Know if I'm Gonna Have a Job"}

NTT faculty face a variety of challenges with stability. The researcher found that NTT faculty would like to know what to expect both in the content of their courses as well as in the number of courses they will be teaching. The nature of the work for part-time NTTs is variable. Part-time NTTs at this university may teach one to three courses a semester on an as-needed basis. The flexibility is what is attractive for the university and some NTTs. The tension is introduced with NTTs being unaware of the number of courses they will be asked to teach consistently and consecutively from one semester to the next. Knowing how many courses you will be teaching in a timely way, longing for stabilization or consistent contracts encompass the subtheme stability. For example, Dan can go for long periods without being asked to teach a course. He recalled a long stretch with no courses, but currently has a full-year contract.

Some of the part-time NTTs shared that they have a desire to become full-time and eventually status NTTs. Maria shared that every semester she hopes to have a full-time appointment. A review of the labor union contract showed one relevant section to changing from part-time to full-time NTT faculty. This section entitled probationary/status (Article XII) outlines that for both full and part-time NTT faculty the probation period is eight consecutive semesters (Agreement, 2017). To account for breaks in employment there is a provision that the eight semesters of employment would fall within eight years. After the probationary period, NTTs receive status. This achievement comes with a negotiated pay increase, change in evaluation 
schedule from annual to every third year, formalized grievance procedure, and prioritization in work assignments. Essentially after four consecutive years of work the NTT faculty member has gained stability in knowing they will be offered a teaching assignment if an NTT faculty position is needed. Maya longed for consistency in knowing how many courses she can be expected to teach. Note she is not necessarily asking to be a full-time employee, she is wanting information to be able to plan her schedule and allow for the opportunity to teach. This information would aid in her feeling more valued by the university. She explained,

I think knowing that we always had employment would help. Uhm being told OK you know we were going to give you all at least one section a semester or however many you know like I think that like consistency would definitely help.

Vanessa reiterated that she needs to know if she is going to have a job to be able to plan financially. She referenced the Gappa and Leslie (1993) categories of Freelancers and Career Enders. She stated these types of NTTs may be able to be more flexible with shorter notice of appointment, but that as a professional courtesy she would like to know what to expect in teaching assignments.

They can't like guarantee us jobs because it's all based on numbers and need and everything but just any sort of, what's the word, about stability. Maybe any sort of stability rather than you know, semester to semester. I don't know if I'm gonna have a job. You know, I don't. I am a mom, I have kids like, I need to work, I need to know that I'm going to have a paycheck next semester. You know, so I think something. I mean, most adults do need that in their life, unless they are the retired or like the freelancer, you know, the different people you mentioned. 
In addition to knowing the number of courses to expect NTTs mentioned consistency in types of courses to be taught as a desire for stability. Maya described bouncing back and forth between courses with different content. She is frustrated that she doesn't have consistency in class assignments to be able to develop her courses. She explained she wants the opportunity to improve in her trade,

uhm, you know really gaining some momentum with that class and then they switch and I know that there are like other people in different sections that wanted to teach because I know you become friends with some of the NTTs and we are like can't we just swap. Like it gets confusing in that regard so I just wish uhm if there was like some consistency with that so that I could develop or kind of hone in my teaching for a specific course.

Teaching professionals strive to improve courses from semester to semester. NTTs that lack stability are not afforded the consideration of a teaching professional to have the opportunity to improve the courses they teach. Maya perceives that if she was valued, she would be given this opportunity. Vanessa also equated stability with value. She says, And that, I mean, for me, it gets into like job stability, it will be really nice to know, like, hey, you're teaching five classes, you're working your butt off for us. But this semester, I'm going to give you four credit hours, like, that's what happened to me last year. And that's terrible. So, like, that's a whole nother story, but like just having some internal feeling of value in the department.

Vanessa explains that receiving consistent and consecutive course assignments is viewed as a type of stability that sends the message of value and competence. Another way to show appreciation or value for work done is through recognition. 


\section{Recognition: "I Just Want to Be Loved"}

When asked what could support their work as educators, interviewees that responded with statements of acknowledgment that they are doing a good job were themed as recognition. This study found that NTTs want to be acknowledged for the work that they do. This includes teaching, but also service involvement on campus and work outside of the classroom with students such as advising clubs. Gretchen included recognition in her desired supports.

I think recognition of the things, all the things that NTT's do. I think obviously not just myself, but a lot of NTTs tend to do service and that's not recognized. And we do it because if we didn't do it, it wouldn't exist for the students.

Gretchen notes that with the large number of NTTs on campus that provide service to students beyond classroom teaching and beyond the NTT position requirements students would miss experiences without the NTTs involvement. Being recognized for tasks outside of the job expectation seem reasonable to Sara. She explained that she often doesn't currently feel valued, and recognition could help.

I just sometimes, I just don't feel valued. So, if you value me, I'm not asking you know for astronomical things you know even if you value me a little bit, I feel like there will be small changes that could be made... I don't know I'd rather someone say, hey look at all these things that you have been doing and the impact that you have made.

Sara argues that small changes are all that is needed to improve in NTTs feelings of importance and appreciation. She went on to explain that she wants recognition not necessarily for teaching, but for the relationships and connections she makes with students. She felt these interactions are crucial for retention and would like to see NTTs receive recognition for the impact they make outside of the classroom. She chuckled and said she sometimes feels like a "life coach" and 
explained it is the little, but time-consuming student interactions that go unnoticed by administration. Sara went on to explain that NTTs service in this realm is uncompensated and not acknowledged. The researcher found the university does offer awards for employees that have an impact on students. NTTs and faculty developers explained that only NTTs that teach general education courses are eligible. The awards referenced are nominated by students for employees that have had a positive influence in their undergraduate program. Receiving recognition was important to a sense of belonging, acceptance, and value for those NTTs that mentioned these awards. It was noted that the awards are currently for general education faculty and thus NTTs that teach upper-division or major-specific courses would not be eligible. The NTTs that requested recognition as a desired support, did not list general education courses within their typical course load. Jada characterizes the recognition she needs to feel valued as love. She released a long hardy laugh and declared loudly,

I just want to be loved (laughter) you know what I mean? I just want to be recognized.

And not me, the NTT role. I think it's just a different type of support for the NTT today. Beyond recognition for her accomplishments, Jada verbalized what others have said about being 'Just NTT' or about not feeling like part of the team. She wants the position of NTT faculty to be acknowledged for its own merits. She wants NTTs to be valued and viewed as equal professionals, not the same as their TT colleagues, but not less than.

\section{Better at My Job}

The last area of supports that would aid in NTTs feeling valuable is those that would assist the NTTs in performing the functions of their jobs. This sub-theme encompasses a desire for supports that directly relate to improvement in the role of an NTT faculty member's position 
responsibilities. Items that were included in this definition were references to acclimation, assessment, and opportunities for growth.

NTTs and administrators alike felt two main things could contribute to the successful acclimation of new NTTs to campus, formal onboarding procedures and assigned mentors. The suggestion of adding a formalized orientation or onboarding process that included clear expectations of responsibilities as well as explanations of procedures and faculty governance at the departmental level was seen as a method to help NTTs acclimate quickly to their environment. This was reinforced by the NTTs in Department B that attended an optional orientation session designed for acclimation purposes. The NTTs that attended demonstrated an understanding of procedures and a connection to the university that aided in their becoming accustomed to the environment. Isabella felt an orientation could include department-specific expectations and explanations of responsibilities that are implied but not currently explicitly stated. She also felt evaluations and the labor union should be discussed.

Yeah, I think like an onboarding experience would be great I think learning about like what you said the governance and opportunities for NTT to serve but with an explanation of what's required and what's not. and not even what's required but what is suggested right.... I think also just letting us know how our evaluations are used and what that what that means because I know that I think the other thing is when I hear about NTT's being evaluated or how easy it like what contract look like for different NTTs like none of that has ever come to me formally.

Isabella is wanting an onboarding process for future NTTs that is purposefully designed to include NTTs in the departments through clear communication of expectations. She adds that none of this information has been formally presented to her, she used various parts of the NTT 
network. The implication is that if NTTs were valued by the institution then formal onboarding would be provided. Formalized onboarding processes were suggested by administrators as well. Administrator, Pat said the onboarding process for NTTs should be like that of the TT faculty and include mentorship.

Formal mentorship was requested as a desired support by NTT faculty as well. This relationship was described as a departmental mentor. A point of contact beyond that of a program lead or program coordinator. The potential mentor was described as a guide to aid in the general inquiry on nuanced sociopolitical departmental questions. As many NTTs also are practitioners, being assigned a mentor is a common practice in their workplaces. Not having a specifically assigned support peer was viewed as strange. Sara also requested the mentorship be formalized as it is for TT faculty at the university.

I wish that NTT had that just so you kind of had a person rather than just kind of collaborating with you know people in the hallway or different people about different issues uhm weekly we're all very open door but sometimes you also don't wanna feel like oh my gosh I have so many questions I feel like I'm bugging people.

Sara explained formalizing the mentorship gives value and importance to the arrangement versus the current NTT informal network of proximity she terms "bugging people in the hallway." Having a peer to guide you was described as significant to navigating the 'pool' rather than being thrown in the deep end alone. Additionally, this removes the feeling of being a burden on others or relying on chemistry or the randomness of proximity. After acclimation, NTTs requested opportunities to assess their performance and contribute to their sense of value.

While NTTs use student feedback as a tool to inform their teaching, many sought more robust evaluation opportunities including both formative and summative evaluations. Formative 
evaluations were suggested in the form of informal check ins. Suggestions included one-on-ones with the director or program lead to have an exchange midsemester to discuss any concerns or challenges that had arisen. Jakes suggested a biweekly 30-minute telephone check in with program leads. He explained his vision this way,

Was there anything you need? How can we support you? Have you had any changes with your syllabus Is there any other support or materials that you need to accomplish the goals of the class? Something like that would be very beneficial, I think.

Jake rattled off a list of questions that could be asked just to check in with NTTs. These included items related to procedural and pedagogical support. Vanessa agreed that having time to touch base and ask questions would be helpful. She mentioned a desire to have summative feedback at five different points in our interview. The frequency of this suggestion would imply its significance to her. One of her statements demonstrates her surprise at the lack of oversight. You know, you just come and go and it's like, people don't really talk to you. But also like, nobody knows if you're here or not.... I'm responsible for this college course and nobody's checking up on me. Okay. You know, that was kind of a surprise.

Vanessa, like so many of her NTT peers, was surprised at how different the university environment is compared to other workplaces. She wanted to be held accountable for the important work she does. NTTs had a desire for substantive summative evaluations. Overall, the NTTs that received evaluations felt they were perfunctory. There was a desire for summative evaluations to provide feedback on how one may grow in their teaching. Again, the implication was a lack of feedback meant they were not valued by the university. Jake described his desire to improve through participation in a feedback process of observation and specific directions for practice change. 
Uhm, I think that that would be a great piece and then really following up with the instructor you know within the week to see you know these are the things that I enjoy that you were doing. This is how you conveyed the information, here are some pieces to work on. Or here are some more things to celebrate and then those can be tied to a formal eval. Because I am one of those people, I feel like you should always want to continue to grow and we are talking to [students-modified to maintain confidentiality] about learning how to keep mastering your craft. It never ends. We should be doing the same in higher ed. So, I think that there should be some type of evaluation schedule that is established and set up because it's the only way to follow through and have some effectiveness within the program.

What Jake described is closing the loop. He wants an evaluation to continue in growth. $\mathrm{He}$ mentioned that having specific items to improve would aid him in selecting professional development. Lastly, he could demonstrate improvement in a future evaluation through documentation of attendance at professional development. These are all items that contribute to professionalism and would contribute to his value in the department.

NTTs desired opportunities to grow in their disciplines through continuing education and on-campus specialized professional development relevant to their unique needs. Continuing education included travel funding for discipline-specific conferences and external professional development opportunities. Gretchen viewed this as an investment in her as an NTT faculty member and felt it was her number one desire for support. Jada agreed and connected professional development opportunities with value. She explains,

Yeah, so I think that is in the decision that the NTT has just as much to offer the students. If they are just as quote-end-quote worthy then they should also be able to receive you 
know that uhm support. Continuing education to improve their teaching or what have you as well. So, I think it should be the same for both for both.

Jada's desire for continuing education support was emphasized with an if then statement. She asserted that if NTTs are valuable, like their TT colleagues, then they too should receive support to continue growing as professionals. The NTTs in Department A do receive funding for professional development, however at a lower rate than their TT peers. NTTs in Departments B and C can apply for funding through a department-level grant. Receiving different amounts of supports is a reminder of the hierarchy and reinforces the stigma of being 'Just NTT'.

Internal professional development specific to NTTs was a desired support to assist with growth. Separate training just for NTTs offered at times that fit their divergent schedules and on topics unique to NTT were desired. This was mentioned not only by the NTT faculty but also by faculty developer, Sue, she says,

But we could offer programming or support that focuses on the special needs, say, instructors who are teaching or working full-time and just teaching one night class a semester, or something like that.... How to balance being a part-time instructor with other responsibilities.

NTTs shared a desire for pedagogical-specific training such as refreshers on educational theory and how to write lesson plans. Additionally, classroom management and baseline information developmental stages of college students. The campus Faculty Development center does offer programs on pedagogy open to both NTT and TT employees. Given what has been discussed regarding the campus culture and hierarchy NTTs may not feel comfortable attending development in shared space with TT colleagues, some of which view NTTs as subordinate. Additionally, NTTs shared that as a first-time NTT faculty they were not aware this on-campus 
faculty development was open to them. Gina shared that she struggled to learn the campus culture in knowing if she could attend workshops provided by the Faculty Development Center, she wanted to feel more confident that she could attend.

...more information about the (Faculty Development Office) training that would have done an official like, we get emails, but I didn't know if I was allowed to go like if those were just for the tenure-line or if that was for the non-tenure line. I didn't know what I was(chuckle). Until a lot (emphasized) later that I realized, oh, those are open to me too. Gina was unclear if the emails marketing workshops from the Faculty Development Office pertained to her. This is not uncommon, since NTTs are often told to ignore parts of emails or information at meetings that are intended for TT only. This finding suggests that it may be beneficial to have professional development specific for NTTs. Isabella felt having NTT specific pedagogical training as a part of the first few weeks of a semester could be helpful to assist with acclimation, understanding, and acceptance at the university.

In this study, NTTs shared desired supports that would not only help them in their current positions but also help newly hired NTTs acclimate and integrate into the university, their department, and all aspects of their positions. These supports focused on being included and valued, having stability and consistency in contracts, being recognized for work done beyond teaching, and the desire for orientations, mentors, and faculty development.

\section{Conclusion}

This chapter has discussed four key themes within the data. The first theme was that of the NTT Network. Lacking formalized support, NTTs created their own methods of acclimating to the university and their roles on campus. These informal networks are forged from necessity and take the form of friendships, proximity, chemistry, self-reliance, and a bond formed from 
being in a similar situation. It is the connection to others that was important in NTT support. The theme of identity: things aren't what they seem demonstrated that NTT faculty filter their professional identities through a complex web of sociopolitical factors. These factors that impact identities the perceived role of NTTs, being an alumni or essential Specialist, and include feelings of value, having a voice, and the power dynamics present which varies by department. The theme of evaluations, or the lack of, produced results demonstrating that student feedback is used heavily, however, NTTs often lack substantial administrative feedback to guide their practice and are unsure how evaluations are used to determine retention. The last theme addressed the support NTTs desire to feel valued by the university. The results, however, are not exclusive to pedagogy but also to items that would aid NTTs in feeling more valuable such as recognition, contributing to the team, onboarding, and professional development. This finding suggests that the teaching role of NTTs requires more than pedagogical support. These four themes interrelate to suggest a need for change in the current university model of NTTs as a subordinate group with an identity of less than operating under unclear expectations and limited contribution and power. The following chapter will relate the findings to the current literature and suggest implications for the findings. 


\section{CHAPTER V: DISCUSSION}

\section{Introduction}

NTTs represent $50-70 \%$ of the professoriate in U.S. higher education and teach up to $54 \%$ of the courses at 4-year public institutions (Bergman, 2011; Haviland, Jacobs, Alleman \& Allen, 2020; Hensely, 2016; Holler, 2014; Kezar, 2014). While NTTs teach the bulk of the undergraduate courses at universities, they are often lacking in areas of support such as orientations, mentor programs, and detailed evaluations (Champlin \& Knoedler, 2017). Current research on NTTs has focused on workplace inequities and the exploration of how the inversion of the professoriate occurred. Discussions on the inversion center around seeking the cause of the increased reliance on NTT faculty concluding benefits to the institution using flexible, specialized, and affordable faculty (Baldwin \& Chronister, 2002; Zhang \& Liu, 2010). Current research regarding workplace inequities focuses on pay deficiencies, lack of resources, exclusion, and poor working conditions for NTTs (Levin \& Shaker, 2011; Ott \& Cisneros, 2015).

There is a growing body of knowledge that demonstrates the lack of institutional and departmental support for NTTs (Davis, 2017; Kezar \& Maxey, 2016; Kezar, 2012). However much of the literature is quantitative and thus is a limited account missing the perspective of the NTTs themselves. Existing qualitative studies are limited to a handful of studies with a specific scope. For example, Levin and Shaker (2011) interviewed full-time NTT faculty in English Departments to explore role identification. Fuller, Brown, and Smith (2017) began to bring NTTs into the conversation by including NTTs as authors through case study contributions. Haviland et al. (2020) expanded what is known about NTTs in a two phases study involving interviews with both NTT and TT faculty and administrators regarding views of collegiality. The present study goes further by providing a holistic view of the experiences of both full and part-time NTT 
faculty and includes perspectives from administrators, union representatives, and faculty developers. The term holistic is used to encapsulate components of the experience of being an NTT faculty member including teaching but also intimately interconnected with the sociopolitical aspects of the NTT position. In the current literature, NTTs are primarily left out of the conversation regarding their roles. The study aimed to give NTTs a voice by answering the following research questions:

RQ 1: How pedagogically prepared did NTT faculty feel when they began teaching at the postsecondary level?

1a. What types of previous experiences or pedagogical training inform NTT perceptions of preparedness?

RQ 2. How do NTT faculty describe the pedagogical support they receive in their teaching roles?

2a. What is the role of professional development in supporting NTT faculty teaching?

2b. How are evaluations used to inform NTT faculty teaching and retention?

2c. What additional supports do NTT faculty desire to improve their teaching? Using Grounded Theory methodology, the present study evaluated artifacts and twenty-three semi-structured interviews including administrators, faculty developers, labor union representatives, and NTTs from three distinct disciplines. This resulted in four major themes: The NTT network, identity things aren't what they seem, evaluations: what's the point, and value: it's not all about money. This chapter contains interpretations of the findings in relation to previous research, implications for practice, and recommendations for future research. Additionally, limitations in the study are addressed. 


\section{Interpretation of the Findings}

While this study began by evaluating NTTs' perception regarding pedagogical preparation, it became apparent during the first few interviews with NTTs that the experience of being an NTT, within the context of the institution studied, is about much more than teaching. The experience of being in an NTT faculty position in U.S. postsecondary education involves not only pedagogical supports but also procedural knowledge and sociopolitical implications. Grounded Theory allows the researcher to develop explanations based on the data gathered and was used in this study to understand the NTT experience at this university (Corbin \& Strauss, 2015). The data from this study revealed that NTTs at this university were lacking formal preparation from the institution regarding procedural and sociopolitical knowledge of their positions and full inclusion as members of the professoriate. The NTTs create an informal 'NTT network' to acclimate and integrate into university life. Further, their identity and roles at the university are complex. While all NTTs in the study related to the 'identity of less than' some, essential Specialists, and alumni, experienced a perception of privilege among their fellow NTTs. The NTTs described the support they received as well as support that was desired to feel valued as a member of the professoriate: being included, having stability, and receiving recognition for their contributions. NTTs also discussed the role of professional development and evaluations in relation to their teaching and the sociopolitical environment. These findings both affirm and furthers the current research on NTTs.

Scott's (1990) terms 'public transcripts' and 'hidden transcripts' serve as an aid in communicating the way dominant and subordinate parties interact in public spaces and private spaces respectfully. These interactions are layered with social nuances that can be difficult for 
outsiders to observe or understand. To explain the 'public transcripts' and 'hidden transcripts' discussed in this study an analogy of carpeting and floorboards will be used. Consider the 'public transcripts' as the carpeting in a room. This is a layer or covering to the wooden floorboards below. The carpet is what is seen by all in the room, even the casual observer. The floorboards then are the 'hidden transcripts.' These floorboards are the true foundation that is supporting the individual standing in the room, not the carpet. The floorboards are the foundational beliefs underneath the carpet. The casual observer would not think to pull back the layer of decoration to view the true structure beneath. It is these floorboards that were exposed in this study through document review and interviews with NTTs, administrators, union representatives, and faculty developers. It is the floorboards that need discussing.

Figure 2: Carpet and Floor Visual Representation Analogy Public and Hidden Transcripts ("Carpet pulled back", n.d.)

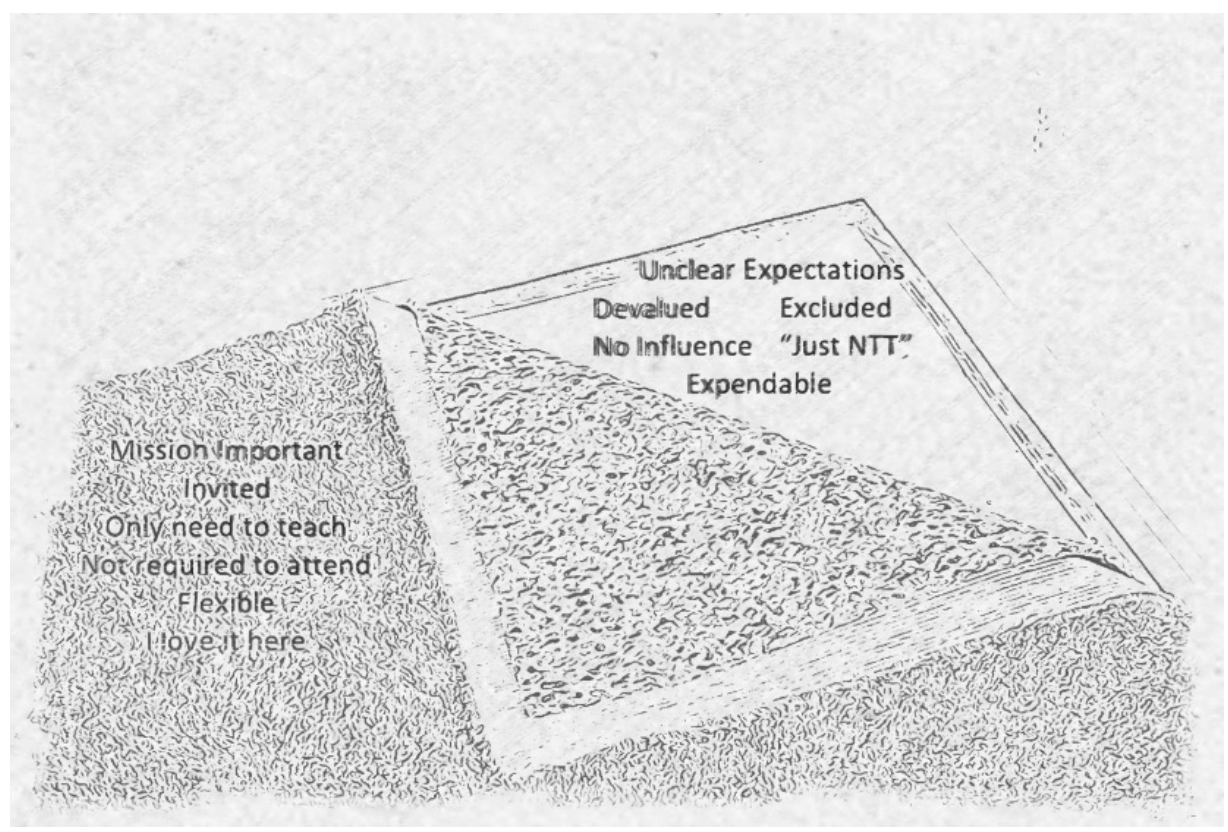


Figure 2 depicts a drawing of a carpet pulled back to reveal the floorboards underneath. This analogy helps to visualize public and hidden transcripts. Publicly NTTs receive messages that they are important to the mission, invited, hired only to teach, not required to attend meetings, and praised for being flexible. Publicly the NTTs in this study discussed openly how much they love their jobs. Pulling back the carpet reveals the hidden transcripts that were shared in confidence. These included unclear expectations, exclusion from departmental decisionmaking and meetings, feelings of being devalued and expendable and having no influence as well as being viewed as 'just an NTT'. This analogy helps to explore the experience and sociopolitical environment of the NTT participants in the study. This study lifted the edge of the carpet to view the floorboards underneath, capturing a glimpse of the NTTs world that includes both public and hidden transcripts. Each theme that emerged is discussed in detail in the following section in relation to the existing research.

\section{The NTT Network}

In answering questions about pedagogical preparedness NTTs in this study discussed support and perceived level of preparation as a whole, rather than limiting perspectives to teaching methods and practice, termed pedagogy. This study's conclusion that NTTs use a network to acclimate to their environment brings a new perspective that has not been discussed in the literature. While previous research shows that NTTs are often not provided resources and adequate pedagogical or procedural support to succeed in their roles such as onboarding, mentoring, professional development, and substantive evaluations (Davis, 2017; Fuller, Brown and Smith, 2017; Hensely, 2016; Hoeller, 2014), none of the researchers have asked how then the NTTs acclimated and integrated to the campus environment. This study adds new information that begins to fill a significant gap in the research by revealing that lacking 
formalized orientations, mentoring and ongoing resources, NTTs create an informal network to fulfill their needs. This network is forged from relationships and connections created from preexisting friendships, proximity, self-reliance, as well as NTTs teaching other NTTs.

This study confirmed what others have found, that NTTs often do not receive formalized orientation and are often lacking resources to complete procedural tasks (Hart, 2011; Kezar \& Sam, 2010; Ott \& Cisneros, 2015). However, this study furthers the research by discovering the methods NTTs are using to compensate despite the lack of formalized orientations. While the literature is clear that NTTs lack onboarding, none of the literature reviewed discussed how NTTs acquire the required skills to succeed in their positions. All NTTs in this study shared stories of using an informal network to acclimate and integrate. The phenomenon of NTTs teaching NTTs is of particular interest because it demonstrates the comradery, empathy, and understanding that NTTs share in having experienced a lack of support themselves. NTTs want to help one another. Forming a network to support one another is similar to what Putman and Kriner (2017) describe as a community of practice. The community of practice is essentially a group of NTT faculty that met in a doctoral seminar course and acknowledged the need for mentorship due to the isolation that is often felt among part-time NTTs. Fuller, Brown, and Smith (2017) advocated for the use of peer mentoring and community building among NTTs to avoid isolation. Haviland et al. (2020) concluded that collegiality is essential for NTTs and a healthy future of postsecondary education. The concern found in this study is that, without formal onboarding and mentoring the information passed down from one NTT faculty member to another may not be accurate or may pertain to one department policy but not in another in which a friend works. Further, the current method of the NTT network relies on chance to ensure acclimation to the institution. This method neglects the NTTs that lack a connection with a friend 
or do not have an office with a helpful colleague. In this study, the NTTs that participated in the optional university and departmental orientations voiced confidence in understanding policy, procedure, and sociopolitical influences. Further, the NTTs that participated in the acculturation orientation had a deeper connection with the institution and its culture. Additionally, failing to have consistent and formalized onboarding and mentoring reiterates to NTTs that their role at the university is insignificant to the institution. These findings suggest that formalizing the informal NTT network through a peer mentor program and consistent orientations would aid in building a much-needed community of support for NTTs as well as provide opportunities to clearly communicate expectations such as evaluations and procedures for rehire. Bringing attention to how NTTs are currently using the network provides the groundwork for administrators and faculty developers to initiate change. If the university wishes to invest in the future of the professoriate, it should invest in NTTs as they are being relied on to provide the bulk of undergraduate education and succeeding despite having inadequate support.

\section{Identity}

The emphasis on identity in this study is situated in the work of Gappa and Leslie (1993). Gappa and Leslie coined the terms Aspiring Academic, Freelancer, Specialist, and Career Ender to classify NTTs as a heterogeneous group with varying motivations. Much of the early literature treated this group as homogenous and was deficit-based in the sense that the researchers viewed the presence of NTTs as harming postsecondary education (Umbach, 2007; Kezar \& Sam, 2010). Additionally, the practice of classifying NTTs by title has been confusing in the research as so many unique terms are used to label NTTs (Berry, 2005; Kezar, 2012). Having NTTs selfidentify in one of the Gappa and Leslie categories framed the exploration into NTT faculty identity within the present study. Understanding identity can aid in choosing appropriate 
supports, rather than assuming all NTTs in every department want the same things. Muncaster (2011) found that having an awareness of the faculty at all levels can be useful in planning how to support them. This study concluded that the NTTs did fit within the framework of Gappa and Leslie. However, the identity of NTTs held a deeper, nuanced meaning for participants. All the participants in this study agreed the role of NTTs was to teach. One interesting difference is in the way individuals characterized their roles.

For example, NTTs in Department A felt their role stemmed from their specialized knowledge as practitioners; they teach from experience. However, those in Department B identified as filling in when TT faculty are not available to teach courses. Finally, Department C NTTs perceived their reason for hire is to teach general education courses or courses that TT faculty did not want to teach. This is consistent with what Baldwin and Chronister (2001) found with NTTs teaching primarily introductory courses with large numbers of students. Scott and Danley-Scott (2015) affirmed that NTTs are teaching the bulk of undergraduate general education courses. Further Kezar and Gehrke (2014) found that hiring NTTs frees up TT faculty to focus on research or other projects. Indeed, this study found NTTs that perceived their role as relief workers for TT faculty and found NTTs that viewed the NTT role as a substitute for TT. What is interesting is the NTT's perception of their role was consistent with the administrators' view of the NTTs in the respective departments. Administrators communicate messages to NTTs that establish a culture in their departments pertinent to the purpose of NTTs. Further, the NTTs in Department A had an identity of belonging and value, stating that they were hired as content experts that bring experience to their teaching. They are involved in curriculum decisions and department meetings and are often tasked with service responsibilities. Being included in their 
department on this level reinforces their identity, however, as Sarah stated this prestige does not carry over to the broader context of the university where she feels like a 'peon'.

Using Scott's (1990) hidden and public transcripts, this study emphasized the importance of both in understanding the sociopolitical environment. Triangulation with administrators, labor union representatives, and faculty developers aided in the insight that NTTs hold an 'identity of less than'. This certainly is not a new finding, several authors affirm the lower status of NTTs in the hierarchal system of the professoriate (Kezar \& Maxey, 2016; Levin \& Shaker, 2011). There is a hierarchy inherent in higher education due to the definitions that distinguish TT and NTT faculty (Kezar \&Maxey, 2013). The language was originally designed to describe the various roles of the faculty, it now holds a cultural implication of dominant and subordinate. The university system has not changed its terminology and structures of separateness have helped to perpetuate the myth that NTTs are less than TTs positions (Ott \& Cisneros, 2015). Levin and Shaker (2011) explored full-time NTT English faculty experiences and found that NTTs had conflicted identities. They concluded that NTTs are not regarded in the same class as their TT colleagues. Certainly, all NTTs want to be valued as professionals, rather than be marginalized to a less than category. Ott and Cisneros (2015) found that full-time NTTs desired a more professionalized work environment consistent with their continuous employment status. Conversely, in this study, NTTs that teach general education courses and do not teach courses in a specific major conveyed stories of being treated more negatively than their NTT colleagues that teach major-only courses. This suggests that the hierarchy present within higher education may have additional layers beyond the two-tiered system of TT and NTT faculty. Levin and Shaker also discussed negative associations with the "nomenclature of the non-tenure track" (p.48). Davis (2017) concluded the use of language serves to perpetuate concepts of NTTs as 
separate and unstable. This is consistent with the finding in this study that NTTs were described by themselves and others as 'Just NTTs'. In the hierarchal system NTTs face reminders that by not having a TT position, they hold less power, prestige, and stability. Every NTT participant interviewed shared experiences of exclusion, marginalization, or disregard for their professionalism.

However, they also shared times of inclusion and it is this information that is unique to this study. Finding practices that do make NTTs feel an accepted part of the professoriate is significant in that this information could be duplicated. For example, this study discovered two identities of NTTs that provide additional insight into perceptions of value and inclusion. These are alumni and essential Specialists. Alumni and essential Specialists are regarded more highly than other NTTs, adding a layer to the already existent hierarchy in the professoriate. The alumni and essential Specialists' preferential treatment are new information not currently found in the literature.

Alumni, who have a connection to the university add to their identity of NTT faculty by also being an alum of the institution in which they now work. The result is these NTTs are both more confident in their abilities to navigate the university procedures and sociopolitical environment due to experience as students. They are often held in higher esteem by their colleagues. Juanita, for example, shared that she was accepted as a colleague to the level of becoming friends and having dinner at the homes of TT colleagues. Other NTTs in her department are excluded from these diner invites because they do not have a preexisting relationship as an alum. Her having been a student, brings her privilege. Haviland et al. (2020) emphasized the significance of inclusion in social and personal interactions for NTTs to feel connected and build trust among colleagues. They found that even simple conversations about 
the weather held importance. Imagine the significance of being invited to someone's home or the awareness that your NTT coworker was invited but you were not. The connection of being an alum is an avenue to develop camaraderie, trust, and a relationship in a department that may otherwise be exclusionary. Ten of the fifteen NTTs interviewed were alumni and explained that navigating campus systems and procedures was easier as a result. This is not surprising as alumni would have some knowledge of course management systems at least from the student viewpoint. Additionally, there appears to be a bond in working for your alma mater.

The other privileged group among NTTs is that of Specialists with expert knowledge from certifications and specialty training beyond the basics of their academic degrees. These NTTs are viewed as professionals on par with their TT colleagues due to their highly skilled knowledge. Using the Gappa and Leslie (1993) categories, seven (47\%) participants identified as Specialists defined as NTTs with discipline-specific expert knowledge. Three NTTs revealed an essential level of training beyond that of the expert knowledge of Specialists. Additional training or certifications brought a sense of job security and prestige to their identity that others in the study did not voice. For example, Gretchen confidentially stated her position is essential for the continuation of the academic program. In short, essential Specialist NTTs know they have additional credentials that are required for accreditation or teaching knowledge in their specific programs. While this additional certification is not in the form of an advanced degree meriting a TT position, it is still valued above NTTs without such credentials. This finding was significant in providing depth of understanding of the NTT identity. Fuller, Brown, and Smith (2017) call researchers to further define distinctions in the Gappa and Leslie (1993) categories and the discovery of alumni and essential Specialist is new information that adds depth to what is currently known about NTT identities. 
The NTTs in this study are represented by a labor union, which is typically viewed as a protection of rights and assuring job security. However, the NTTs on this campus are either ambivalent or unaware of the labor union and the contractual agreement with language specific to retention and evaluation. The labor union agreement helped interpret the negotiated intent of evaluations. That intent is stated as developing NTT faculty as professionals. However, the stability gained through the contract and the guidelines surrounding the evaluation procedures are not being attributed to the labor union by the NTTs, but rather to Human Resources. The confusion seems to stem from Human Resources implementing and managing evaluation procedures. Since many of the NTTs were unaware of the union contract that stipulates evaluation processes and course assignment criteria used in rehire, NTTs are relying on the NTT network to determine what behaviors are most adventitious in seeking continued employment. Certainly, not all NTTs seek to be full-time, Career Enders, for example, are retirees, however, having clear information on the number of courses that will be assigned and if they will have a position from one semester to the next provides stability. While the labor union was not found to be influential in the identity of NTTs, gaining the negotiated longevity benefit of status was as it provided this stability.

Status is essentially an eight-semester probation period for NTTs. This is a negotiated benefit available at the university in this study. Having obtained status had a large impact on NTT's stability as it essentially serves as a method to have automatic contract renewal from year to year. Ironically, many part-time NTTs who wished to be hired full-time were not aware of the union or this negotiated benefit. This is consistent with Fuller, Brown, and Smith's (2017) discussion of organized labor and NTTs, that while the conversation around unionization is growing, the actual numbers and participation in labor unions is waning. Unfortunately, there 
was insufficient participation from the labor union representation to discuss further insights in this regard.

\section{Evaluations}

Evaluations are a tool to be used for informing teaching practice, but also as a form of assessment for retention and rehire. Despite their importance, research shows few NTT faculty receive substantive feedback or any evaluations (Bland et al, 2006; Kezar, 2013b). Evaluations at this institution are a compilation of two separate processes: administrative and student feedback. The first type of evaluation of NTTs stems from anonymous feedback from students gathered at the end of each semester. The NTTs in the study overwhelmingly agreed that they read all this information, sift for patterns, and change their course delivery and teaching methods as a direct result. Modifying practice from feedback demonstrates that not only do the NTTs want feedback but that they will use it to inform their practice. Further, NTTs in this study assumed that positive student evaluations would influence administrative evaluations and rehire decisions.

The second type of evaluation was administrative. The results of this study demonstrate that administrative evaluations for NTTs were compliance-driven. Administrators are conforming to negotiated policies and rules when implementing evaluations but have largely missed the purpose of evaluations to foster NTTs' development and growth. Consistent with the findings in this study, Kezar (2013b) conveys stories of administrators going through the motions by providing critiques to meet the required assessment for NTTs without substance, resulting in evaluations in name only. Thirteen of the 15 NTTs interviewed said that administrative evaluations were perfunctory. NTTs shared stories of form letters, submitting duplicate materials from previous years, receiving no substantive feedback, and lack of follow-through after observations. Additionally, $60 \%$ of the NTTs interviewed did not know how evaluations 
were used to determine retention. $33 \%$ of NTTs interviewed felt evaluations were not used for retention and only one person had a positive view of the process and perceived a direct link between evaluations and retention. These numbers are staggering and are evidence that while the process of evaluation is occurring the purpose is not clear to the NTTs. The reality is at this institution, evaluations are not being used for development or hiring decisions. Administrators confirmed that hiring decisions were primarily based on need and personal interactions with the NTTs. Administrators relied on student feedback, hallway interactions, and overall feel for an NTTs demeanor when dispensing reappointments.

While the process of evaluation is occurring, the purpose is unclear to the NTTs. Being unsure of the evaluation criteria to ensure continued employment, NTTs perform tasks beyond the scope of teaching in hopes that this will be seen in a positive light and earn them favor with the administration. For some NTTs this is serving on committees, advising clubs, or accepting last-minute teaching contracts. For others, this included attending professional development. Professional development was seen as both a means and an end, with some attending to grow professionally and others attending in hopes it will be noticed. However, as one NTT, Sara, bemoaned there was no point in attending professional development aside from personal growth as it had no impact on recognition or evaluation making it difficult to be motivated to attend. NTTs at this university may not be hired back from one semester to the next due to poor performance or low enrollments, in either case, the individual NTTs may be unaware of the cause.

\section{Value}

The deficits discussed above in onboarding, mentorships, evaluations, and role identity connect closely with the items that NTTs felt could aid them in feeling more valued and 
supported in performing the functions of their positions. In previous literature, Kezar (2012) recommended providing supports through improved working conditions, being valued for teaching contributions, and having input into policies through faculty governance to include NTTs throughout campus culture. There are similarities between these recommendations and the items requested by the NTTs, administrators, union representatives, and faculty developers in this study. Much of what was requested did not involve pay increases but rather emphasized being included, valued, having stability in work contracts, receiving recognition for contributions and supports that would aid the NTTs at being better equipped to do their jobs.

NTTs in two of the three departments wanted to be included in departmental meetings, curricular matters that impact the courses they teach, and faculty governance. This inclusion goes beyond being present at meetings into being a contributing member of the team, consistent with Baldwin and Chronister (2001). Kezar (2012) recommends NTTs be involved in faculty governance at multiple levels. This act legitimizes the NTTs as professionals that can contribute to the work in higher education, beyond substitute teachers. NTT faculty may have a seat at the table but not feel welcome to sit down. Haviland (2016) found that NTTs felt limited agency and respect in the workplace and ultimately want to have a voice in campus decisions at multiple levels. The outlier in this study was the NTTs in Department A who did say they felt included at this level within their department, but not outside their department at the dean or university level. Additionally, these NTTs were aware of the uniqueness of their inclusion and involvement and felt at times the expectations of service were unspoken and uncompensated. This suggests that while NTTs want to be included, participating beyond the expectations of the teaching role should be clearly communicated and recognized in some manner. Kezar (2012) also noted the need for NTTs to have clearly defined job expectations. 
NTTs held a sense of being unimportant or devalued as was noted in the earlier theme 'Just NTT.' This 'identity of less than' was so prominent that every NTT interviewed referenced it in some way, as did the administrators, faculty developers, and union representatives. It is of no surprise that the desired support of being valued would be the most often stated need from the NTTs. The perception is that value will come through having stable employment contracts, recognition, and support such as orientations, mentors, and professional development. Davis (2017) discussed the dualistic orientations of NTTs as prestigious experts in the classroom and feeling diminished in their professional capacity among colleagues. This speaks to a desire to be valued. Davis' work further advocated for material equity, described as not only pay but also position security as a means for workplace improvement. These suggestions would aid in NTTs being considered important, worthwhile professionals who are valued colleagues. Haviland et al. (2020) concluded that NTTs currently lack and have a strong need for collegial experiences and environments that include trust, respect, and recognition of professional expertise.

Further, previous researchers agree with the finding that this vulnerable group longs for stability. As Kezar (2014) noted, NTTs are hired and not continued based on enrollment. This allows the administrators to make last-minute flexible hiring decisions but creates instability among the NTTs wanting information on what topics to prepare to teach and if they need to seek employment elsewhere. This study revealed stories of NTTs asked to teach a course within one day of the start of the semester. There needs to be a balance in being flexible, but also allowing adequate time to complete the needed tasks of the profession. Thompson (2003) discussed that this vulnerable group can face nonappointment without justification. This partners with the desire NTTs have for perceived value through stability, clear expectations, and substantive evaluation. Stability is a desire from a practical view of planning for life. Davis (2017) explains 
that NTTs do not need to be fired, they can simply not be re-hired. He further calls rehire the most important part of job insecurity. This study reaffirms Davis' findings that stability is a desired support that contributes to perceptions of value.

NTTs and administrators alike mentioned formal onboarding, mentors, and professional development as methods to communicate inclusion, value, and support to NTTs. Orientations are currently available at the university level but are optional and often NTTs are unaware of the option to attend. This speaks to a disconnect in communicating this needed information. Additionally, orientations are only available in the fall semester, making it impossible for spring semester hires to attend. The recommendation is to formalize orientations specifically for NTTs at the university level into electronic or virtual formats that would allow NTTs to attend. This is the same request that was voiced by NTTs regarding professional development. Several authors found that professional development may be available to NTTs but may not be offered at times that are conducive to their schedules (Baldwin \& Chronister; 2001, Keezar, 2012). For example, an NTT faculty that is a Specialist holding another full-time job outside the university or the NTT Freelancer working for multiple institutions could find it challenging to fit the orientation into their schedule. In addition to variant times of day for onboarding and faculty development, the NTTs in this study mentioned a desire for professional development separate from their TT colleagues. The faculty developers interviewed emphasized that NTTs and TT are treated alike and used statements demonstrating that they do not distinguish between the two. However, in an attempt to be fair to NTTs, the faculty developers may in fact be missing an opportunity to provide training specific to NTT needs. Tapp and McCourt (2017) state that faculty developers and administrators lack "audience awareness" when it comes to understanding the needs of NTTs (p.96). They go on to explain that professional development for NTTs needs to consider 
the unique circumstances such as overwhelming course loads, time management, and lack of funding for professional development. The NTTs in this study requested faculty development to meet their specific needs including opportunities to connect with NTT colleagues. Levin and Shaker (2011) found that professional development not only aids in developing skills, but also contributes to a sense of belonging, professionalism, and value. Additionally, Kezar (2012) suggested orientations and ongoing opportunities for professional development as best practices in acclimating NTT faculty to their positions in postsecondary education.

One of the goals of the study was to make recommendations for potential future supports and affirm those methods which are currently perceived as beneficial by the NTT faculty. Recommendations will be discussed in the next section of implications for practice.

\section{Implications for Practice}

The infrastructure of postsecondary education was designed for TT faculty. Positions that were viewed as temporary (NTTs) did not require permanent language or support systems from the institution. However, as evidenced by the rapid growth in the use of NTT faculty, these positions are no longer a temporary solution and thus the infrastructure needs to be modified to include resources to develop NTTs as professionals. Steady employment of NTT positions both part and full-time shows no signs of decreasing. As the profession of NTT faculty has developed, the onboarding, evaluation, and professional development processes have not grown at universities to include this group as a priority. Additionally, NTTs are in a vulnerable position of uncertainty in continued employment contracts contingent upon course demand. This combination creates an environment composed of a large group of employees wanting support and feeling insecure to request assistance. Add to this the sociopolitical nature of a hierarchal 
system of TTs and NTTs where the latter are marginalized as the subordinate group of 'just NTT' and hold an 'identity of less than.'

This research contributes to the body of work on what is known about NTTs and strives to add a qualitative perspective as well as make suggestions for practical application. The results of this study suggest a change in campus culture and practices to embrace the NTT faculty as contributing and not tangential higher education professionals. This concept echoes the work of Kezar and Maxey (2016) who advocate for a realignment of faculty models to ones of scholarly educators. They acknowledge that there is not a one size fits all model, but a move towards professionalism embracing faculty for their various strengths in a broader sense than what is currently occurring in higher education.

To minimize systemic exclusion the sociopolitical barriers must first be acknowledged and then addressed. During this study, NTTs spoke of supports that were helpful with acclimation and inclusion. These best practices will be shared here and should be duplicated across the institution. In this manner, the burden of creating new systems to infuse NTTs into the campus culture can be lightened by embracing those methods that individual departments are already doing well. Four areas will be highlighted to begin the hard work of campus climate change: acceptance and inclusion, onboarding and professional development, expectations and evaluations, and lastly mentorship. Improvements in any of these areas will assist the university in embracing and developing the professionalism of the NTT faculty. If NTTs are to remain in positions teaching at universities, then adequate pedagogical and procedural supports must be provided as is done for TT faculty. 


\section{Acceptance and Inclusion}

Universities hire NTTs as a part of the faculty to deliver the number of undergraduate courses in demand at their institutions, however, they do not fully include NTTs into the university culture. Acceptance is defined as being admitted to the group. Inclusion, however, was defined by the participants in this study as having access to resources, opportunities to contribute to the group, and being valued by their coworkers as participating members of the community. The current hierarchal system establishes NTTs as subordinate; thus, contribution is limited and reinforces the 'identity of less than.' It takes time to change a culture and promote community integration. There is no one size fits all solution to systemic exclusion of NTTs. Overall universities must invest resources in exploring the specific needs unique to NTTs at their campuses by having focus groups to gather information and enlisting advocates to change the segregated environment. An NTT Resource Office needs to be developed to allow NTTs a safe place to request assistance, lodge concerns, and collaborate with other NTTs. Due to the diverse needs of NTTs, the following recommendations should be considered in the sociopolitical context of individual departments and include perspectives from NTT faculty.

Attitudes and language Attitudinal barriers are inaccurate beliefs or perceptions about a person's ability often based on stereotypes. While attitudes are not changed with policies, those in power can influence attitudes as role models and advocates in what they promote and what they permit. Administrators must promote an environment of respect for all faculty regardless of rank. One way attitudes are expressed is through the use of language. The use of inclusive language can begin to reframe how NTTs are viewed by their TT colleagues. For example, NTTs need to be introduced as the specialists they are. Their accomplishments and departmental contributions need to be included in public spaces such as faculty meetings, newsletters, and 
bulletin boards alongside their TT peers. Additionally, the terms colleagues, faculty, and professoriate must be used in an inclusive sense to mean both TT and NTT workers. Administrators must have an intolerance for the use of terms that marginalize or imply a less than quality, for example 'just NTT' or 'that NTT'. At a minimum introduce people by their names. One NTT faculty member in this study was deeply hurt that coworkers did not even know their name. As advocates and allies, administrators, and TT colleagues in positions of power and influence can support NTTs by affirming their equity and speaking up on their behalf. Administrators can consider adopting language that describes what the faculty member does, rather than what they lack (tenure status). Terms such as Freelancer and Specialist would promote the use of accurate language that presents NTTs as the professionals they are and conveys an environment of collegiality and respect.

Invite Another way to include NTTs is through an invitation to both work-related and social activities. It needs to be clearly communicated to NTTs when they are included and when they are not, explaining what is optional and what is expected. Giving NTTs a choice is the best option when considering involvement. The researcher recommends inviting NTTs, explaining the involvement and level of commitment of an activity explicitly stating if the involvement is expected. This transparency helps avoid misunderstanding and implications of additional work for no pay. It also avoids feelings of exclusion or confusion. NTTs must be invited to faculty meetings and committees that impact the courses they teach. Changes should not be decided for NTTs without the direct participation of members of the group affected by the changes. NTTs in Department A appreciated the inclusion in faculty meetings and felt more connected to their department and colleagues as a result. Further, if there are items that pertain to TT faculty only a 
separate meeting needs to be held to cover these items. Talking around NTTs is exclusionary and akin to having a party and ignoring half of the guests.

Basic resources NTTs must be given dedicated office space and have access to conference rooms, computers, and photocopy equipment to perform the tasks of their jobs. Physical access to required materials and spaces is a basic need for employees. NTTs in this study mentioned that they would enter a room and be unsure if they were allowed to even be in the space. Clearly identified office space with a name placard on the door and inclusion on the departmental website are outward symbols of connection and belonging to the group as well as professionalism. Similarly, if business cards, name tags, and logo clothing are provided for newly hired TT faculty as welcome gifts, these items need to be available to NTTs as well. Having tangible items that label the NTTs as a member of the group conveys belonging, comradery, and shared meaning. These small gestures will aid the NTTs in feeling comfortable and not segregated in a diminished faculty category.

Involvement Include NTTs as a part of the educational community by involving NTTs in decisions that impact their work in real and meaningful ways as contributing professionals. For example, three NTTs in this study shared frustrations that the courses they have taught for many years had curriculum changes without their input. While the NTTs were invited to the meetings, they had no real power to impact change and thus were not truly involved. This resulted in the NTTs incorporating a new curriculum that they neither supported nor did they feel connected or invested in. Excluding the NTTs in these decisions, resulted in resentment towards the TT faculty and the curriculum itself. Conversely, three other NTTs specifically mentioned contributing significant information towards curriculum changes and shared perceptions of value that their expertise was sought. This experience contributed to their integration and acceptance 
into the academic community. Seeing as the NTTs teach a majority of the undergraduate courses, their involvement in the curriculum must be valued and will provide distinct insights that are currently being ignored. NTTs need to be encouraged to provide input into curriculum, syllabi content, selection of textbooks, assignments, and assessments of the courses they teach. Changes should not be decided for NTTs without their direct participation. While NTTs with variant schedules may not be able to attend meetings at traditional times, involvement needs to be solicited through surveys, focus groups, or virtual means.

Another way to begin to correct the deficit culture in postsecondary education is by involving NTTs on committees or in projects that have influence and power in their departments and allow the NTT faculty to showcase their expertise. This positions the NTT faculty member as a content expert and reframes the idea of what NTTs can contribute to the educational environment as more than substitute teaching. Again, as this is additional work for the NTTs, appropriate recognition, reward, or compensation needs to be provided for this work. NTTs in key leadership positions can be granted release time for their additional efforts. Alternatively, NTTs can be contributors by being guest speakers in TT colleagues' courses or being a contributor during a faculty meeting. For example, during departmental meetings, NTTs need to be invited to add insights and have input in the same manner as the TT faculty. These steps of involvement not only esteem the NTTs but also add to a culture of inclusive professionalism by giving opportunities for NTTs to demonstrate their contributions and value. Public recognition of NTTs serves to send messages that NTTs are valued as key members of the university.

True inclusion can be a self-perpetuating method to change the sociopolitical environment. NTTs that contribute by being asked for their input and being listened to are more likely to contribute in the future, thereby demonstrating their value to the group. TT faculty that 
has positive interactions with NTTs as contributing coworkers are more likely to view NTTs as members of the educational community and develop a culture of acceptance. A vital part of inclusion is being a contributing member of the department and being recognized for contribution. Haviland et al. (2020) found that departments that nurtured an environment of trust and respect and acknowledged professional expertise were the ones in which TT and NTT faculty felt included and sense of collegiality.

Inclusion best practice example This campus could learn from the NTTs in Department A that stated they could contribute to decision making, curriculum, and department-level service opportunities. Overall, these NTTs felt included. While the NTTs in Department A are a good model for being included through contribution, it was not without tensions. The difficulty lies in the fact that these contributions are not acknowledged with a reward system nor are they always clearly communicated in position expectations. Therefore, those in this department viewed them as additional uncompensated work. To truly be included, NTTs must be encouraged to contribute and be valued as a member of the team as well as be acknowledged or compensated in some way for their contributions. The researcher acknowledges that inclusion is a lofty goal in a system with a historical perspective of NTTs being separate and not equal. Broad systemic changes can be time-consuming, but providing resources and respect are a starting point to gaining professionalism for this vulnerable and needed group.

\section{Onboarding and Professional Development}

Orientations are a method to introduce the expectations and culture of a community. Omitting NTTs from departmental and campus orientations contributes to the isolation, exclusion, and confusion NTTs shared as part of their 'identity of less than'. Onboarding and training NTTs will establish consistent campus and departmental expectations, provide accurate 
information and resources, and contribute to defining NTTs as professionals. Similarly, professional development unique to NTTs will serve to fill any gaps in knowledge or misinformation because of the NTT network functioning as an informal onboarding method. Providing professional development opportunities broadens skills and encourages educators to expand their craft. To grow in a profession focused on the role of teaching, development needs to be provided in the arena of pedagogy. The Faculty Development Center on this campus is already offering many services to equip faculty in their careers, however, as NTTs in this study noted, it was not clear if NTTs could attend. Further, training was often offered at times NTTs were unable to attend. Faculty developers must explicitly market services to NTTs and include training at flexible times of the day and in virtual and prerecorded formats to accommodate variant schedules. Additionally, the university must reevaluate the methods of communication among departments to ensure the department and university-level orientations and professional development are available and communicated to all NTTs each semester.

What alumni NTTs teach us Discovering that alumni felt more connected and acclimated to the university was unexpected. However, this information provides valuable insight to the university. What made alumni feel more connected was the comfort of knowing campus procedures and culture. This suggests that participating in campus and department-level orientations along with a cultural orientation can be beneficial for all NTTs. Alternatively, to streamline, information regarding campus culture can be included at the departmental level orientation which appeared more relevant to NTTs than the university-wide orientation. Participants that attended the optional university acculturation orientation stated they had a clear understanding of the university's mission, campus services available and felt more connected to the institution. This is like the experience of alumni who have an established sense of belonging. 
The information contained in the acculturation orientation included strong messaging about the individual's value to the whole. The orientation communicates not only what the university believes but also how an individual can be a part of the mission, vision, and culture. The messages communicated at this orientation are ones of inclusion and belonging. By infusing culture into orientations specifically for NTTs, these faculty can be included in the organizational beliefs and values and be more connected as members of the educational community.

Mentorship Another area of professional growth involves ongoing guidance and learning from a colleague. The NTTs are currently mentoring one another from a sense of empathy. Having been new themselves and lacking a mentor, they are willing to mentor other NTTs. By taking advantage of the phenomenon of the informal NTT network, a formal mentor program must be developed that encourages acclimation and inclusion. Mentors must be trained to ensure accurate information is conveyed among NTTs. Full-time status NTTs would be excellent choices for mentors. These individuals have been at the university for an extended period and understand the unique sociopolitical environments of their departments. However, individual departments need to assess the most appropriate NTTs to be leaders.

Program coordinators/course leads who are providing various levels of guidance to NTTs must also be trained and tasked with some level of mentorship. These positions have leadership in their programs and are often approached to provide additional procedural or pedagogical support. The recommendation is for training and formalizing the mentor relationship between the coordinator/course lead and NTT faculty. Training coordinator/course leads can address issues of inconsistency within and across departments. Program coordinators/course leads serve as important connections by building community with NTTs. This can be done with once a 
semester check in. Additionally, recognition or compensation needs to be provided for this responsibility.

Formal mentorship programs are common as a part of new TT faculty hires onboarding programs and include stipends for both the mentee and the mentor. NTT faculty must be compensated similarly for the work they are already doing. Mentor tasks can include providing information about campus resources, advice on grappling with difficul classroom management and pedagogical questions, and encouragement for professional growth. Adding mentoring to the NTTs onboarding process can add to the professionalism of being an NTT faculty member and contribute to the development of a university culture of inclusion. Mirroring the TT onboarding and mentoring processes for the NTTs further communicates a sense of value to not only the NTTs but also other employees at the institution.

Onboarding best practice example Department B has successfully offered an NTTspecific, department-level orientation for new hires. This should be duplicated by other departments as well as made available at variant times of day/evening or in flexible formats to ensure all NTTs have access. This training goes beyond a tour of the department and provides information on the department priorities, procedures, and information on-campus resources. In addition to providing much-needed information, this orientation communicates to NTTs that their position is important in the department and is worthy of assimilation. Further, this orientation provides evidence that NTTs are a needed part of the department. This type of messaging is needed to begin to change the culture of less than. This orientation can be expanded to provide opportunities for NTTs to socialize and form networks with one another and their TT colleagues. 
Professional development best practice example Department B has also provided professional development for NTTs that was positively received. This type of professional development should be offered university-wide and provide not only content knowledge but also community opportunity for NTTs to collaborate. Department A provides travel funds for NTTs to continue discipline-specific development. While this may not be financially feasible for all departments, marketing the free opportunities available through the Faculty Development Center will not only improve NTT's knowledge through continuing education but also aid in creating an environment with clear messages of inclusion where NTTs are positioned as valuable enough to the university to invest resources. While the current literature focuses on faculty development provided by centers, department-level training can be catered to the unique needs that arise in an individual department. Departments are also uniquely positioned to understand the needs of their faculty as well as their schedules. For example, following classroom observations for evaluation a trend may appear in needing training on a specific pedagogical technique. Of course, department administrators can also stay abreast of the faculty development opportunities on campus and recommend these.

Formal onboarding and professional development can minimize the need for the NTT network to acclimate NTTs to the university, thus providing means for accurate information and adding to the professionalism and inclusion of NTTs through the investment of time and resources.

\section{Expectations and Evaluations}

Evaluations provide crucial information to inform teaching practice and determine reappointment. However, on this campus, administrative evaluations were found to be perfunctory in nature. Having unclear expectations and limited feedback creates an insecure 
work environment for NTTs. The presence and quality of evaluations varied widely among departments.

NTTs must receive regular scheduled administrative evaluations inclusive of teaching observations with specific feedback on how to improve their trade. It also needs to be communicated that items such as professional development, on this campus, only help the individual to grow and do not directly relate to continued contracts. Evaluators must receive training on pedagogy or consider enlisting the assistance of professionals in the Department of Education to have clear observational protocols. A coaching model can be implemented for feedback, allowing collaboration between the NTT faculty member and the evaluator. Form letters were viewed as impersonal and need to be avoided. Feedback needs to include connections to professional development opportunities on campus to encourage growth. For example, evaluators can discuss the benefits of class formats of lecture versus the inclusion of hands-on activities and suggest pedagogical workshops that can aid in developing the necessary skill to teach using these methods. Maria discussed the importance of her evaluation in that was able to incorporate feedback into an action plan including pursuing further academic readings and professional development to grow her teaching practice.

Explicit communication Another item that must be communicated at department-level orientations to NTTs are clear expectations of duties, such as what will be reviewed during evaluations and which items are factors when considering reappointment. NTTs may be unsure of expectations of their positions outside of teaching. Again, this is in part due to the lack of formalized onboarding. In addition, the criteria to be used in the evaluation and how these evaluations are used to determine retention aids in minimizing the need for the NTT network and can lead to an improved sense of stability for NTTs. While NTTs may hope their attendance at 
professional development or service on committees sways their departments to rehire, at this campus, the reality is appointment is primarily based on need. This information may not be clearly communicated to NTTs. Without clear information, NTTs experiment with theories shared amongst each other as to what is expected. One of the theories that exist in the NTT world for ensuring continued employment is to make yourself valuable by contributing to the department through service as is the case with the NTTs in Department A discussed earlier. Another theory developed by NTTs to demonstrate competency and possibly gain favor toward continued employment is through attendance at professional development. Communication must be transparent with NTTs about position expectations, evaluation purposes, and the process for rehire. As administrators in this study mentioned, they use their general feeling of the NTT to determine to rehire, it is difficult to tell the extent which participating in non-teaching duties impacts rehire.

For full-time NTTs on this campus, acquiring status provides security of reappointment and is based on longevity. There must be explicitly communicated policies outlining reappointment procedures for NTTs. This information needs to be available in multiple formats, through the website, and at orientations. The department can provide statistics on the conversion of appointments from part-time to full-time and from full-time to status appointments. The Gappa and Leslie (1993) categories can be utilized to identify the types of NTTs and their desired level of appointment. Career Enders for example may be content teaching one or two courses to allow for flexible schedules. Conversely, Aspiring Academics by nature are seeking TT appointments. Availability of these opportunities, or lack of, should be clear. Transparency and open communication are recommended to develop an educational community of respect. 
Expectations and evaluations best practice example As an example of best practice, Department A has an appointment, salary, promotion, and tenure policy document for NTTs clearly outlining the evaluation expectations. This document was voted upon by the NTTs employed at the time of its inception, however, due to the transient nature of NTT faculty, the document needs to be reviewed and provided to new hires during orientation. Notably, the NTTs in Department A did not mention this document, it was provided by the administrator. As a result, the researcher could not determine if the NTTs in Department A were aware of the document. Each department needs to develop a similar document to outline expectations for service, meeting attendance, and information on how evaluations are used to determine retention.

Further evaluations that include observations and substantive feedback with opportunities for improvements add to the professionalism of NTTs. Department B currently has a procedure for NTT evaluations that includes a pre-observation form that the evaluator can use to determine the NTT's teaching philosophy and professional goals. Frontloading the evaluation includes the NTT faculty as an active member in their evaluation and allows the opportunity for selfreflection on pedagogy. Department B also meets with NTTs to discuss results and encourage professional development as a method for informed teaching. While this reflective process was not used consistently with the NTTs in Department B, it is a starting point to developing more substantive evaluations that NTTs can then use to inform teaching and grow as professionals. Other departments need to consider adopting evaluations that include both observations and reflective opportunities for growth.

The practical implications of this study demonstrate there is much work to be done to stop the systemic exclusion and marginalization of NTT faculty in postsecondary education. The 
next section provides suggestions for further study that can contribute to understanding this diverse group.

\section{Recommendations for Future Research}

Little research has been done that center on the voices of NTTs. Follow-up research should explore the NTT faculty perspective in many areas of their experience. One such area that was mentioned in this study was that some full-time NTTs are conducting research and performing service duties for the university. These tasks are often uncompensated, which begs the question: why? Why would individuals that are often viewed in low esteem and that have large course loads consider giving even more time to the university to serve on committees and to develop research? NTTs in this study were of unaware how their evaluations were used to determine retention. As a follow up it should be determined if this was unique to this campus or is a phenomenon in a larger sense. A study to determine the motivations behind actions that are not a required aspect of the position such as service, research, and curriculum development could add perspective to what is currently known about NTTs. While an interesting topic, it was beyond the scope of this current project and is being left for others to explore.

The current study should be expanded to include NTT faculty from other universities of similar size and focus to provide more data for comparison. The Gappa and Leslie (1993) categories would prove beneficial in comparison on a national level and adding more to the cases would increase transferability. Further research into the identities of NTTs could evaluate the hierarchy within the NTTs themselves. Additionally, the two identities unearthed in this study, alumni and essential Specialists, should be evaluated to determine if these can deepen the Gappa and Leslie (1993) categories and further bring understanding to this heterogeneous group. The 'NTT network' should be dissected to discover how NTTs learn to connect and what occurs if 
NTTs do not avail themselves of these informal resources. Perhaps they no longer continue teaching and will be an evasive group to explore.

The apathy towards the labor union and lack of involvement by the NTTs on this campus is curious considering the push for organized labor nationwide. A study exploring NTTs' perceptions and involvements in labor unions locally and nationally would add a unique perspective to the growing body of literature. A comparison of NTTs' perceptions on power and privilege from those who work at institutions with vibrant and involved organized labor groups and those who do not would serve to provide practical information to the role of unionization. This aspect was impossible to include in the current study due to limited participation from the labor union leadership.

A study to compare NTT and TT faculty attitudes toward evaluation, professional development, student, and administrative evaluations would prove interesting and expand what is currently known about the two groups. Are the two groups similar in perceptions and attitudes about these topics, or are there differences that stem from the unique professional roles? Lastly, future work that examines the nexus of perceptions of NTT and TT in terms of value and contribution to begin to change the culture is necessary to end the marginalization of this valuable group.

\section{Limitations}

Every study has limitations. This study's limitations include scope, timing, positionality, and sample. This study was limited in scope. While the NTT participants in the study came from three diverse departments, they all came from the same institution and thus would be influenced by culture and expectations common to that region. Additionally, the study did not include any R1 or private institutions. Each institution has different working conditions and attempting to 
generalize or make assumptions that all NTTs have the same experience would be too simplistic. It would be interesting to duplicate the study in a broader scope and compare NTTs by institution type. The timing of the study is also a limitation as the interviews took place during the Covid19 world pandemic. This limitation impacted the interview format. One interview was face to face and the remainder took place over telephone or zoom. Much can be communicated through body language and thus this was lost in the telephone interviews and difficult to interpret via virtual meetings. Additionally, the searcher did request that participants consider procedural and pedagogical support needed pre-pandemic, but considering the interviews took place in the middle of a pandemic, answers were most likely influenced by this situation. A probe was added to the NTT interviews to inquire if the pandemic impacted the types and level of supports needed for NTTs. No new information emerged because of the question. A limitation in positionality is noted in that the researcher is currently a full-time NTT faculty member and while guarded against bias, cannot fully remove their own experience from the research.

The sample also had limitations. The researcher interviewed only one administrator in each department. The administrator in Department A was interim and while they had worked as faculty and administration in Department A prior to becoming a director they had limited experience in the evaluation of NTTs. Further, broadening the scope of interviews to include TT faculty and additional levels of administration could have brought deeper knowledge and perspectives. Only one member of the labor union agreed to an interview making it difficult to include perspectives from this important group. The researcher relied on labor union artifacts and the one interview but acknowledges this is a very limited perspective. Regarding faculty development, the researcher became aware that there are faculty development offerings at the dean's level in some of the departments. Since none of the NTTs mentioned these development 
opportunities these faculty developers were not contacted. Lastly, while the NTT faculty sample was sufficient to reach saturation it was not evenly balanced in full or part-time status. NTTs in the study included 13 (86\%) full-time and three (14\%) part-time. Thus, the full-time perspective had a higher likelihood of influencing this study.

\section{Conclusion}

Through semi-structured interviews and evaluation of artifacts, this study was able to add to the growing body of research on NTT faculty. This study was undertaken to give voice to a vulnerable group. Using Grounded Theory methodology, the researcher developed explanations based on the data gathered. This dissertation has discussed four key themes within the data. These four themes interrelate to suggest a need for change in the current university model of NTTs as a subordinate group with an 'identity of less than' operating under unclear expectations and limited contribution and power. NTTs are complex with identities that go beyond the marginalized view of 'just NTT'. The hidden transcripts of 'just NTT' are mixed with department-specific sociopolitical implications. The current university system privileges some over others. Clear expectations with training, mentorship, and quality evaluations are needed to allow the individuals in these positions to be judged for their merits and not for being 'just NTT'. Having high expectations for the role is possible. For change to occur it will not only take a structural modification but also a cultural shift in how NTT work is perceived. The hidden transcripts must be made known. The words of administrator Alex helps to summarize this study, many, many, many of our core sections are taught by non-tenure track faculty members. So, to ignore their professional growth, to ignore their professionalism, to ignore their connectedness to us, would be to kind of abandon our mission because they're just many 
of them and they they [sic] need to be a part of us to make sure they're carrying forward with our, our mission.

As Alex explained the university cannot continue to exclude NTT faculty and ignore their professionalism.

This study has implications for administrators, faculty developers, and NTTs. Implications for administrators through gaining knowledge of how NTTs felt at hire, the supports they received, and what they see lacking, stakeholders can create a support scaffold to better meet the needs of this heterogeneous group to perform the tasks required in their jobs. Faculty developers can use this study to gain a better understanding of the unique needs of this new majority and turn to offer professional development in flexible formats and at variant times. Additionally, topics can focus on building community among NTTs and topics that meet their unique needs. For example, training on time management, large course loads and class sizes, and information on campus-specific policies and procedures. NTTs themselves will benefit from this study only if those in power make the suggested changes. Mehta's (2013) work on power dynamics explains that how problems are framed impacts not only the solutions pursued but also the power distribution of who has a voice in solving the problem. Historically the problem has been viewed as too many NTTs are working in higher education. Perhaps the problem is that the system of higher education has not changed to keep up with the needs of the new faculty majority.

Universities must consider a new model to support the changed professoriate, one with collaboration and support for and with both professional NTT and TT faculty. The professoriate has changed from a hierarchical system of TT and NTT faculty to one inclusive of a variety of employees hired to meet the employment needs of the university. Some are hired for research, 
others service, and still others to teach. Whereas some faculty are expected to perform all three roles, others are not. This does not make their work less significant. Nor does it mean they are not as qualified to do their work or unworthy of adequate support systems. NTTs have persisted on university campuses despite a separatist system of marginalization that insists on reducing their profession to 'just NTT'. This research suggests a need for a community collaboration model of distinct positions, each defined, valued, and supported for their own merits collaborating to provide excellence in postsecondary education. A change in the faculty model requires a change in campus culture to foster an environment of inclusion. Gradually the professoriate has inverted to an NTT dominant faculty, it's time the university support systems catch up. 


\section{REFERENCES}

Adams, K., (2002). What colleges and universities want in new faculty: Preparing future faculty occasional paper series (7). Washington, D.C.: Association of American Colleges and Universities. Retrieved from https://eric.ed.gov/?id=ED472499

Ahn, R. (2018) Balancing the equation: Does a Ph.D. equal expertise in teaching? International Journal of Teacher Leadership 1 Volume 9, Number 2. Retrieved from https://eric.ed.gov/?id=EJ1202346

Agee, J. (2009). Developing qualitative research questions: a reflective process, International Journal of Qualitative Studies in Education, 22:4, 431-447, doi: $10.1080 / 09518390902736512$

Alleman, N., \& Haviland, D. (2017). "I expect to be engaged as an equal”: collegiality expectations of full-time, non-tenure-track faculty members. Higher Education 74(3), 527-542. doi:10.1007/s10734-016-0062-4

Amundsen, C., Abrami, P., McAlpine, L., Weston, C., Krbavac, M., Mundy, A., \& Wilson, M. (2005). Proceedings from Annual Meeting of the American Educational Research Association. The what and why of faculty development in higher education: An in-depth Review of the Literature. Montreal, Canada.

Ary, D., Cheser Jacobs, L., Sorenson, C., \& Razavieh, A. (2010). Introduction to research in education ( $8^{\text {th }}$ edition). Wadsworth Cengage Learning, Belmont, CA.

Baldwin, R. G., \& Chronister, J. L. (2001). Teaching without tenure: Policies and practices for a new era. Baltimore, MD: Johns Hopkins University Press.

Baldwin, R. G., \& Chronister, J. L. (2002). What happened to the tenure track? In R. P. Chait (Ed.), The questions of tenure (pp. 125-159). Cambridge, MA: Harvard University Press. 
Baldwin, R. G., \& Wawrzynski, M. R. (2011). Contingent faculty as teachers: What we know what we need to know. American Behavioral Scientist, 55, 1485-1509. doi:10.1177/0002764211409194

Banasik, M. \& Dean, J. (2015). Non-tenure track faculty and learning communities: Bridging the divide to enhance teaching quality. Innovative Higher Education, 41, 333-342. doi: 10.1007/s10755-015-9351-6.

Berger, R. (2015). Now I see it, now I don't: Researcher's position and reflexivity in qualitative Research. Qualitative Research, 15, 219-234. doi: 10.1177/1468794112468475

Bergman, D. (2011). A Study of adjunct faculty. (Unpublished doctoral dissertation). Montana State University.

Berry, J. (2005). Reclaiming the ivory tower: Organizing adjuncts to change higher education. New York, NY: Monthly Review Press.

Bland, C., Center, B., Finstad, D., Risbey, K., \& Staples, J. (2006). The Impact of appointment type on the productivity and commitment of full-time faculty in research and doctoral institutions. Journal of Higher Education, 77, 89-123. doi: 10.1353/jhe.200.0002

Bolman, L., and Deal, T. (2013). Reframing organizations: Artistry, choice, and leadership. John Wiley \& Sons, Incorporated. Retrieved from https://ebookcentral.proquest.com

Brown Barnes, C. (2017). Contingent workforce size, characteristics, compensation, and work experiences of adjunct and other non-tenure-track faculty. (Report No. GAO-18-49). Retrieved from https://www.gao.gov/products/GAO-18-49

Bowen, G. (2008). Naturalistic Inquiry and the saturation concept: a research note, Qualitative Research, 8; 137 doi: 10.1177/1468794107085301 
Cahn, S. (2008). From student to scholar: A candid guide to becoming a professor. New York: Columbia University Press.

Carpet Pulled Back [Image]. (n.d.). Modified from https://www.shutterstock.com/imagephoto/pulled-back-carpet-padding-room-house-491386840

Center for Community College Student Engagement (2014). Contingent commitments: Bringing part- time faculty into focus (A special report from the Center for Community College Student Engagement). Austin, TX: The University of Texas at Austin, Program in Higher Education Leadership. Champlin, D. P., \& Knoedler, J. (2017). Contingent labor and higher education. Review of Political Economy, 29, 232-248. doi:

10.1080/09538259.2017.1316054

Conrad, L. Y., \& Tucker, V. M. (2019). Making it tangible: hybrid card sorting within qualitative interviews. Journal of Documentation, 75(2), 397-416. doi: 10.1108/JD-06-2018-0091

Corbin. J., \& Strauss, A. (2015). Basics of qualitative research: Techniques and procedures for developing grounded theory. Thousand Oaks, CA: Sage.

Creswell, J. and Creswell, J. (2018). Research design: Qualitative, quantitative, and mixed methods approaches. Thousand Oaks, CA: Sage Publication Inc.

Cross \& Goldenberg (2009). Off-track profs: Nontenured teachers in higher education. Cambridge, Mass.: MIT Press.

Davis, D. (2017). Contingent academic labor: Evaluating conditions to improve student outcomes. Sterling, Va: Stylus Publishing. 
de Saxe Zerden, L., Ilinitch, T. L., Carlston, R., Knutson, D., Blesdoe, B. E., \& Howard, M. O. (2015). Social work faculty development: An exploratory study of Non-Tenure-Track women faculty. Journal of Social Work Education, 51, 738-753. doi:

$10.1080 / 10437797.2015 .1076284$

Eagan Jr., M. K., Jaeger, A. J., \& Grantham, A. (2015). Supporting the academic majority: Policies and practices related to part-time faculty's job satisfaction. Journal of Higher Education, 86(3), 448-483. doi: 10.1080/00221546.2015.11777371

Feldman, D., \& Turnley, W. (2001). A field study of adjunct faculty the impact of career stage on reactions to Non-Tenure-Track jobs. Journal of Career Development, 28, 1-16. https://doi.org/10.1023/A:1011193713662

Finkelstein, M., \& Schuster, J. (2011). A new higher education: The next model takes shape. New York, NY: TIAA-CREF Research Institute.

Fuller, R., Brown, M., \& Smith, K., (Eds.) (2017) Adjunct faculty voices: Cultivating professional development and community at the front lines of higher education. Sterling, Va: Stylus Publishing.

Gappa, J., Leslie, D. (1993) The invisible faculty: Improving the status of part-timers in higher education San Francisco, CA: Jossey-Bass.

Gehrke, S., \& Kezar, A. (2015). Supporting Non-Tenure-Track faculty at 4-year colleges and universities: A national study of deans' values and decisions. Educational Policy, 29, 926-960. doi: 10.1177/0895904814531651

Goulding, C. (1998). Grounded Theory: The missing methodology on the interpretivist agenda. Qualitative Market Research: An International Journal, 1(1), 50-57. doi.10.1108/13522759810197587 
Hallberg, L. (2006) The "core category" of grounded theory: Making constant comparisons. International Journal of Qualitative Studies on Health and Well-being, 1(3), 141-148. doi: $10.1080 / 17482620600858399$

Hansen, K. (2013). College instructors' preparedness to teach students with learning disabilities. (Unpublished doctoral dissertation). The University of Western Ontario.

Hart, J. (2011). Non-tenure track women faculty: Opening the door. The Journal of the Professoriate, 4(1), 96-124. Retrieved from https://search-ebscohostcom.libproxy.lib.ilstu.edu/login.aspx?direct=true $\& \mathrm{db}=\mathrm{a} 9 \mathrm{~h} \& A N=69886786 \&$ site $=$ edslive \&scope $=$ site

Hativa, N., Barak, R. and Simhi, E. (2001). Exemplary university teachers: Knowledge and beliefs regarding effective teaching. The Journal of Higher Education, 72, 699-729. doi: $10.2307 / 2672900$

Haviland, D., Jacobs, J., Alleman, N. and Allen, C. (2020). Inclusive Collegiality and Nontenuretrack Faculty. Sterling, Va: Stylus Publishing.

Hensley, B. (2016). Adjunct faculty in a Neoliberal Age: The power of critical stories. (Doctoral dissertation). Retrieved from Proquest LD2347 .Z98 no.1916eb.

History of the AAUP (n.d.). Retrieved from https://www.aaup.org/about/history-aaup Holler, K. (2014). Equality for contingent faculty: Overcoming the two-tier system. Nashville, Tennessee: Vanderbilt University Press.

Hussein, M. E., Hirst, S., Salyers, V., \& Osuji, J. (2014). Using Grounded Theory as a Method of Inquiry: Advantages and Disadvantages. The Qualitative Report, 19(27), 1-15. Retrieved from https://nsuworks.nova.edu/tqr/vol19/iss27/3 
Jones, W., Hutchens, N., Hulbert, A., Lewis, W., \& Brown, D. (2017). Shared Governance among the new Majority: Non-Tenure Track Faculty Eligibility for Election to University Faculty Senates. Innovative Higher Education, 42(5/6), 505-519. doi.org/10.1007/s10755-017-9402-2

Knapp, L. G., Kelly-Reid, J. E., \& Ginder, S. A. (2010). Employees in postsecondary institutions, fall 2009, and salaries of full-time instructional staff, 2009-10 (NCES 2011150). U.S. Department of Education. Washington, DC: National Center for Education Statistics. Retrieved from http://nces.ed.gov/pubsearch/pubsinfo.asp?pubid=2011150

Kezar, A., \& Holcombe, E. (2015). The professoriate reconsidered. Academe, 101. Retrieved from https://www.aaup.org/article/professoriate-reconsidered\#.W0OlhthKii4

Kezar, A. (2012). Improving contingent faculty relations: Changing campuses for the new faculty majority. New York: Routledge.

Kezar, A. (2014). Changing faculty workforce models. New York, NY: TIAA-CREF Research Institute.

Kezar, A. (2013a). Departmental cultures and non-tenure track faculty: willingness, capacity, and opportunity to perform at four-year institutions. The Journal of Higher Education, 84(2), $153-188$.

Kezar, A. (2013b). Examining non-tenure track faculty perceptions of how departmental policies and practices shape their performance and ability to create student learning at four-year institutions. Research in Higher Education. 54(5), 571-598. doi10.1007/s11162-0139288-5

Kezar, A. (Ed.). (2012). Embracing non-tenure track faculty: Changing campuses for the new faculty majority. New York, NY: Routledge. 
Kezar, A., \& Maxey, D., (2016). Envisioning the faculty for the 21st century: Moving to a mission-oriented and learner-centered faculty model. New Brunswick, NJ: Rutgers University Press.

Kezar, A., \& Maxey, D., (2015). Adapting by design: Creating faculty roles and defining faculty work to ensure an intentional future for college and universities. New Brunswick, NJ: Rutgers University Press.

Kezar, A., \& Maxey, D. (2012). Missing from the institutional data picture: Non-Tenure-Track faculty. In J. X. Yonghong (Ed.), Refining the focus on faculty diversity in postsecondary institutions: New Directions for Institutional Research (pp. 47-65). San Francisco: Jossey-Bass.

Kezar, A., \& Maxey, D. (2013). The changing academic workforce. Trusteeship, 21 (3): 15-21. Retrieved from https://agb.org/trusteeship-article/the-changing-academic-workforce/

Kezar, A., \& Sam, C. (2010). Understanding the new majority of Non-Tenure-Track faculty in higher education: Demographics, experiences, and plans of action. Association for the Study in Higher Education Report, 36(4). San Francisco, CA: Jossey-Bass.

McClenny, K., \& Arnsparger, A. (2014). Contingent commitments: Bringing part-time faculty into focus (A special report from the Center for Community College Student Engagement). Austin, TX: The University of Texas at Austin, Program in Higher Education Leadership. Retrieved from https://www.ccsse.org/docs/PTF_Special_Report.pdf

McCormick, A. C. (2001). The Carnegie classification of institutions of higher education. Menlo Park, CA: The Carnegie Foundation for the Advancement of Teaching. 
McKee, C.W. \& Tew, W. M., (2013). Setting the stage for teaching and learning in American higher education: Making the case for faculty development. New Directions for Teaching and Learning, 133, 3-14. doi: 10.1002/t1.20041

Mehta, J. (2012). The allure of order: High hopes, dashed expectations, and the troubled quest. New York, NY: Oxford University Press.

Merriam, S.; Tisdell, E. (2016) Qualitative research: a guide to design and implementation. San Francisco, CA: Jossey-Bass.

Michel, J. O., Chadi, D., Jimenez, M., \& Campbell, C. M. (2018). Ignis fatuus effect of faculty category: Is the tenure versus non-tenure debate meaningful to students' course experiences? Innovative Higher Education, 43(3), 201-216. doi: 10.1007/s10755-0179420-0

Muncaster, K. (2011). Supporting adjunct faculty within the academy: From road scholars to retired sages, one size does not fit all (Doctoral dissertation) retrieved from http://hdl.handle.net/2345/2426.

National Center for Education Statistics (2018). NCES handbook of survey methods. Retrieved from https://nces.ed.gov/statprog/handbook/pdf/nsopf.pdf

National Center for Education Statistics. (2013). Integrated Postsecondary Education Data System. Washington, D.C.: United States Department of Education.

Oleson, A., \& Hora, M., (2014). Teaching the way they were taught? Revisiting the sources of teaching knowledge and the role of prior experience in shaping faculty teaching practices. High Education 68: 29. https://doi.org/10.1007/s10734-013-9678-9 
Olson, G. (2009) Exactly what is 'shared governance'? The Chronicle of Higher Education. Retrieved from https://www.chronicle.com/article/Exactly-What-IsShared/47065\#comments-anchor.

Ott, M., \& Cisneros, J. (2015) Understanding the changing faculty workforce in higher education: A comparison of full-time non-tenure track and tenure line experiences. Education Policy Analysis Archives, 23(90). doi:10.14507/epaa.v23.1934

Pace, D. (2017). The decoding disciplines paradigm: Seven steps to increased student learning. Bloomington, IN: Indiana University Press.

Piccanio, A. G., \& Spring, J. (2013). The great American education-industrial complex. Ideology, technology, and profit. New York, NY: Taylor and Francis.

Planning, Research and Policy Analysis Illinois State University. (2019). University Factbook 2019. Illinois State University. https://prpa.illinoisstate.edu/downloads/university/2019_Factbook-200214-final.pdf

Poch, R. K. (1993). Academic freedom in American higher education: Rights, responsibilities and limitations. ASHE-ERIC Higher Education Report No. 4. Retrieved from https://search.ebscohost.com/login.aspx?direct=true \&db=eric\&AN=ED366263\&site=eds -live\&scope $=$ site

Putman, P. \& Kriner, B. (2017). Using a Community of Practice to Enhance the Adjunct Experience. In Fuller, R., Brown, M., \& Smith, K., (Eds.) Adjunct faculty voices: Cultivating professional development and community at the front lines of higher education (pp. 62-69). Sterling, Va: Stylus Publishing. 
Ran, F. X., \& Xu, D. (2019). Does contractual form matter? The impact of different types of non-tenure-track faculty on college students' academic outcomes. Journal of Human Resources, 54(4), 1081-1120. doi 10.3368/jhr.54.4.0117.8505R

Reichman, H., \& Scott, J. W. (2019). The Future of Academic Freedom. Baltimore: Johns Hopkins University Press. Retrieved from https://search.ebscohost.com/login.aspx?direct=true \&db=nlebk\&AN=1916486\&site=edslive $\&$ scope $=$ site

Rios, R. (2018). Teacher agency for equity. New York, NY: Routledge.

Schein, E. H. (2016). Organizational culture and leadership, 4th ed. San Francisco, Jossey Bass

Schmid, M., Gillian-Daniel, D., Kraemer, S., \& Kueppers, M. (2016). Promoting student academic achievement through faculty development about inclusive teaching. The Magazine of Higher Learning, (48)5, 16-25. doi:10.1080/00091383.2016.1227672

Schmidt, P. (2013). University adjuncts are often denied a share of shared governance, study finds. The Chronicle of Higher Education. Retrieved from https://advance-lexiscom.libproxy.lib.ilstu.edu/api/document?collection=news\&id=urn:contentItem:59W5H591-DYTH-919W-00000-00\&context=1516831.

Schunk, D. H. (2016). Learning theories: An educational perspective. Boston: Pearson.

Shaw, V. (2004). Career making in postmodern academia. Maryland: Hamilton Books.

Skinner, B. F. (1956). A case history in scientific method. American Psychologist, 11 (5), 221233. https://doi.org/10.1037/h0047662

Sorcinelli, M. D. (2007). Faculty development: The challenge going forward. Peer Review, 9, 48. Retrieved from https://www.aacu.org/publications-research/periodicals/facultydevelopment-challenge-going-forward 
Scott, J. C. (1990). Domination and the arts of resistance. Hidden transcripts. New Haven, CT: Yale University Press.

Scott, G., \& Danley-Scott, J. (2015). Two loops that need closing: Contingent faculty perceptions of outcome assessment. The Journal of General Education: A Curricular Commons of the Humanities and Sciences, 64, 30-55. doi: 138.87.237.178

Stolzenberg, E., Eagan, K., Zimmerman, H., Berdan Lozano, J., Cesar-Davis, N., Aragon, M., \& Rios-Aguliar, C. (2017). Undergraduate teaching faculty: The HERI faculty survey 20162017. Retrieved from https://heri.ucla.edu/monographs/HERI-FAC2017-monograph.pdf Tapp, S. \& McCourt, A. (2017). Creating Faculty Development Opportunities for Adjuncts. In Fuller, R., Brown, M., \& Smith, K., (Eds.) Adjunct faculty voices: Cultivating professional development and community at the front lines of higher education (pp. 9096). Sterling, Va: Stylus Publishing.

The Board of Trustees of Illinois State University and ISUEA/IEA-NEA (2017). Agreement. https://hr.illinoisstate.edu/downloads/Labor_Contract_NTT.pdf

Thelin, J. R. (2011). A history of American higher education. Baltimore, MD: The John Hopkins University Press.

Thompson, K. (2003) Contingent faculty and student learning: Welcome to the strativersity. New Directions for Higher Education 003(123), 41. https://doi.org/10.1002/he.119

Tuckman, H. P. (1978). Who is part-time in academe? AAUP Bulletin, 64(4), 305-315.

Umbach, P. D. (2007). How effective are they? Exploring the impact of contingent faculty on undergraduate education. Review of Higher Education, 30, 91-123. doi:10.1353/rhe.2006.0080 
Waltman, J., Bergom, I., Hollenshead, C., Miller, J., \& August, L. (2012). Factors contributing to job satisfaction and dissatisfaction among Non-Tenure-Track faculty. The Journal of Higher Education 83(3), 411-434. doi:10.1353/jhe.2012.0014.

Weimer, M. (2004). How faculty learn to teach better. The Teaching Professor. Retrieved from https://www.facultyfocus.com/free-reports/effective-group-work-strategies-collegeclassroom/

Zhang, L., \& Liu, X. (2010). Faculty employment at 4-year colleges and universities. Economics of Education Review, 29, 543-552. doi: 10.3386/w21827 


\section{APPENDIX A: INTERVIEW QUESTIONS NTT FACULTY}

\section{Descriptive Information:}

1. When did you first begin teaching in higher education?

2. How many courses are you teaching this term (include all institutions at which you teach)?

a. How many places do you currently work?

b. How many different courses have you taught? In which disciplines?

3. Given the following categories, how would you classify yourself?

a. Career ender: retiree teaching courses to fill in not intended on working full-time.

b. Specialist: NTT with discipline-specific expert knowledge. These individuals may teach full-time or have a career in a related profession and teach contingently.

c. Freelancer: choose to teach part-time for flexible schedules.

d. Aspiring academic: are in search of full-time academic appointments. These NTT may hold terminal degrees and may teach courses at multiple institutions to piece together a wage-earning job.

\section{Understanding the experience:}

1. Thinking of the first time you taught at this institution, how prepared did you feel to teach? (Not at all prepared, a little prepared, prepared, well prepared, very well prepared)

a. What previous experiences or training did you have that impacted this feeling?

2. What support did you receive to help you in your teaching role at the university?

a. Who provided this support?

b. What support would be helpful to you to improve your teaching?

3. Have you- participated in organized professional development opportunities provided by this institution?

a. Can you tell me about your experience? For example, who provided the development, what was the nature of it, and what impact did it have on your position as an NTT faculty member?

b. In your opinion, what is the role of professional development in supporting NTT faculty teaching?

4. Can you tell me about your departmental evaluation process? For example, how often are you evaluated, by whom, and in what ways?

a. How are evaluations used in your department to determine retention?

b. Do you use the results of your evaluation? If so in what ways?

5. Overall, what could be done to better support your work as a teacher?

6. Is there anything else that you would like to add to help me to understand your experience teaching at the postsecondary education?

Follow-up with probing questions related to procedural, pedagogical, or sociopolitical environment impacting the NTT faculty member's ability to integrate into post-secondary education. Request artifacts that would document this experience. (Documents may include evaluation, orientation, promotion, or other) 


\section{APPENDIX B: INTERVIEW QUESTIONS UNIVERSITY EMPLOYEES}

Semi-structured interview questions for university employees that are charged with the task of selection, evaluation, or working closely with NTT faculty at the departmental level

\section{Descriptive Information:}

1. What is your title and role in working with NTT faculty in your department/school? How long have you worked in this capacity?

2. Given the following categories, how would you classify the NTT faculty that work in your department/school?

a. Career ender: retiree teaching courses to fill in not intended on working full-time.

b. Specialist: NTT with discipline-specific expert knowledge. These individuals may teach full-time or have a career in a related profession and teach contingently.

c. Freelancer: choose to teach part-time for flexible schedules.

d. Aspiring academic: are in search of full-time academic appointments. These NTT may hold terminal degrees and may teach courses at multiple institutions to piece together a wage-earning job.

\section{Understanding the experience:}

Thinking of your current department/school in relation to NTT faculty both full and part-time, but not graduate students:

1. What do you look for in an NTT faculty hire? How do you identify those you would like to interview or consider for a teaching role?

2. What types of previous experiences or pedagogical training are preferred for NTT faculty to possess at hire?

3. When NTT are initially hired do they receive a formal campus-wide orientation? If yes, please explain the components. If no, why not?

4. What types of pedagogical support are provided for NTT?

a. Follow-up probes may include: What resources/support are provided to help them improve their teaching?

b. What funds, if any, are available for NTT professional development?

c. Is formal mentoring offered? If so, how is this implemented?

5. What types of support are requested by NTT?

a. Follow-up probes may include procedural, pedagogical, sociopolitical

6. Can you tell me about departmental evaluation process for NTT? For example, how often are NTT evaluated, by whom, and in what ways?

a. How are evaluations used in your department to determine retention?

b. How are NTT faculty informed of their evaluation results?

7. Overall, how could departments better support NTT faculty?

8. Is there anything else that would like to add to help me to understand the NTT faculty experience teaching in your department? 
Follow-up with probing questions related to procedural, pedagogical, or sociopolitical environment impacting the NTT faculty member's ability to integrate into post-secondary education.

Request artifacts that would document this experience. (Documents may include evaluation, orientation, promotion, or other) 


\section{APPENDIX C: INTERVIEW QUESTIONS FACULTY DEVELOPERS}

\section{Descriptive Information:}

1. What is your title and role in working with NTT faculty? How long have you worked in this capacity?

2. Given the following categories, how would you classify the NTT faculty that you work with?

a. Career ender: retiree teaching courses to fill in not intended on working full-time.

b. Specialist: NTT with discipline-specific expert knowledge. These individuals may teach full-time or have a career in a related profession and teach contingently.

c. Freelancer: choose to teach part-time for flexible schedules.

d. Aspiring academic: are in search of full-time academic appointments. These NTT may hold terminal degrees and may teach courses at multiple institutions to piece together a wage-earning job.

\section{Understanding the experience:}

Thinking of your role as a faculty developer in relation to NTT faculty both full and part-time, but not graduate students:

1. When NTT faculty are initially hired do they receive a formal campus-wide orientation?

a. What is covered in the orientation?

2. What types of pedagogical support are provided for NTT?

a. Follow-up probes may include: What resources/support are provided to help them improve their teaching?

b. What formats and times are these offered? (i.e., online, weekend)

c. What funds, if any, are available for NTT professional development?

d. How many NTT typically attend workshops? (Part and full-time breakdown)

3. What types of support are requested by NTT?

a. Follow-up probes may include procedural, pedagogical, sociopolitical

4. Overall, how could faculty development be improved to support NTT faculty?

a. How many NTT's do you typically work with?

5. Is there anything else that would like to add to help me to understand the NTT faculty experience related to professional development?

Follow-up with probing questions related to procedural, pedagogical, or sociopolitical environment impacting the NTT faculty member's ability to integrate into post-secondary education. Probes may include 'can you tell me more about that?' or 'please explain'.

Request artifacts that would document this experience. (Documents may include evaluation, orientation, promotion, or other) 


\section{APPENDIX D: LABOR UNION REPRESENTATIVE QUESTIONS}

\section{Descriptive Information:}

1. What is your title and role in working with NTT faculty? How long have you worked in this capacity?

2. Given the following categories, how would you classify the NTT faculty that are represented by the union?

a. Career ender: retiree teaching courses to fill in not intended on working full-time.

b. Specialist: NTT with discipline-specific expert knowledge. These individuals may teach full-time or have a career in a related profession and teach contingently.

c. Freelancer: choose to teach part-time for flexible schedules.

d. Aspiring academic: are in search of full-time academic appointments. These NTT may hold terminal degrees and may teach courses at multiple institutions to piece together a wage-earning job.

Understanding the experience:

Thinking of your role in relation to NTT faculty both full and part-time, but not graduate students:

1. When NTT faculty are initially hired do they receive a formal campus-wide orientation? If yes, please explain the components. If no, why not?

2. What types of pedagogical support are provided for NTT?

a. Follow-up probes may include: What resources/support are provided to help them improve their teaching?

b. What funds, if any, are available for NTT professional development?

c. Is formal mentoring offered? If so, how is this implemented?

3. What types of support are requested by NTT?

a. Follow-up probes may include procedural, pedagogical, sociopolitical

4. Can you tell me about evaluation processes for NTT? For example, how often are NTT evaluated, by whom, and in what ways?

a. How are evaluations used to determine retention?

b. How are NTT faculty informed of their evaluation results?

5. Overall, how could departments better support NTT faculty?

6. Is there anything else that would like to add to help me to understand the NTT faculty experience?

Follow-up with probing questions related to procedural, pedagogical, or sociopolitical environment impacting the NTT faculty member's ability to integrate into post-secondary education. Request artifacts that would document this experience. (Documents may include evaluation, orientation, promotion, or other) 


\title{
APPENDIX E: INFORMED CONSENT
}

\author{
Informed Consent Form
}

You are being asked to participate in a research study conducted by Rachel Smith in the Department of Teaching and Learning at Illinois State University to evaluate non-tenure track faculty perception regarding teaching in post-secondary education. You must be a current full or part-time non-tenure (NTT) track employee of Illinois State University, university employees that is charged with the task of selection, evaluation, or working closely with NTT faculty at the departmental level, faculty Developer, or NTT labor union representative. If your title is graduate assistant, you are ineligible to participate. You are ineligible to participate if you are currently within the European Economic Area.

\section{Why are you being asked?}

You have been asked to participate because you are either an NTT employee or have import in interacting with NTT employees. Your participation in this study is voluntary. You will not be penalized if you choose to skip parts of the study, not participate, or withdraw from the study at any time. You can also skip interview questions you do not feel like answering.

\section{What would you do?}

If you choose to take part in this research study you will be asked to answer a series of semistructured interview questions by Rachel Smith. The interview will be recorded and transcribed. The interview will last approximately 30 minutes. You will be contacted to complete a follow-up interview. This second interview will be to verify the information collected during the first interview. 


\section{Are any risks expected?}

The primary risks associated with this study are threats to confidentiality and risk to employability or reputation should a breach of confidentiality occur. Please know that all notes and voice recordings used for this study will be kept confidential. Your identity will never be revealed as part of sharing outcomes from the study. To reduce these risks, participants may choose to skip any interview question they do not want to answer.

\section{Will your information be protected?}

All reasonable effort will be made to keep any provided personal information confidential. All information provided will remain confidential and will only be reported as group data, when possible, with no identifying information. Instead, Rachel Smith may use a pseudonym (made up name) to protect your identity. All data collected will be stored in a locked office, under password protected computer file. However, when required by law or university policy, identifying information (including your signed consent form) may be seen or copied by authorized individuals.

\section{Could your responses be used for other research?}

Your identifiable information will not be used in future research, but your deidentified information could be used for future research without additional consent from you.

\section{Who will benefit from this study?}


This study hopes to better understand the NTT experience in relation to pedagogical support from the perception of the NTT employee. As this study focuses on identifying best practices related to NTT faculty, a variety of stakeholders might find results from this study to be useful, including professional/faculty developers, faculty leaders from labor unions, administrators in higher education who are involved in hiring and retaining NTT faculty, and NTT professionals themselves.

\section{Are there incentives?}

Your participation in the study begins with an interview and concludes with a follow-up interview to verify the data collected. Upon completion of the follow-up interview you will receive one \$20 Amazon gift card.

The IRS may consider these payments to be taxable compensation. Recipients of a research participant incentive payment may want to consult with their personal tax advisor for advice regarding the participant's situation. Any participant also has the opportunity to participate in the study without accepting the research incentive payment.

\section{Whom do you contact if you have any questions?}

If you have any questions about the research or wish to withdraw from the study, contact Rachel Smith at 309-438-3668 or resmit1@ilstu.edu. This study is being overseen by Dr. Erin Mikulec emikule@ilstu.edu. 
If you have any questions about your rights as a participant, or if you feel you have been placed at risk, contact the Illinois State University Research Ethics \& Compliance Office at (309) 4385527 or IRB@ilstu.edu.

\section{Documentation of Consent in Person Interviews}

Please sign below if you are willing to participate in this study.

Signature

Date

You will be given a copy of this consent form for your records.

\section{Documentation of Consent Zoom/Telephone Interviews}

Verbal consent to the question: Would you like to participate in this study? 


\section{APPENDIX F: CODE BOOK}

Name

\section{Desire support for value}

Recognition I just want to be

loved

Release Time

Stability I don't know if I'm gonna

have a job

Value It's not all about money

Autonomy

Better at my job

Acclimate

Formal mentor program

Onboarding process

Assess

Nobody really checks in

Real Evaluations

Growth

Continuing Ed and Travel

'better investment'

\section{Description}

References to items that would increase feelings of support. These include recognition and predictable contracts. Inclusion and appreciation as a contributing team member. Acknowledgment of doing a good job. Compensation, awards, or verbal praise.

Time off from coursework for additional duties

Knowing how many courses you will be teaching in a timely way. Longing for stabilization or consistent experiences.

Supports NTT request to feel more supported or valued in their positions.

Feelings of independence and trust in performing the requirements of the job.

A desire for supports that directly relate to improvement in the role of an NTT faculty member's responsibilities.

Onboarding, orientation and mentoring as suggestions for supports that would help new NTT to acclimate to their work environments and responsibilities

Assigned coworker to assist with questions

The suggestion of adding a formalized orientation or onboarding process

Formative and summative evaluations from supervisors

Meetings with supervisors to see how the NTT is doing

Wanting formative evaluations with specific behavioral observations to aid in growth

Desire for Professional learning opportunities.

Travel monies and/or funding for professional development 
Specialized PD 'Oh, those Separate trainings just for NTT. Offered at times that fit their divergent schedules and on topics unique to NTT

are open to me too'

\section{Connection}

Inclusion I want to be a part

of the team

NTT Community building

Value

We Wish We Could Pay Them

More

\section{Evaluations, what's the point}

Above and beyond

Coaching for improvement

Specific helpful feedback

Day to day feelings

Evaluation process transcends

purpose

I really don't know

Low Expectations

NOT

Roll over
Mention of supports that would connect the NTT to their departments or each other

A desire to be included in decision making or departmental happenings. To feel like a part of the team or have representation

Opportunities for NTT to gather for common discussions, connection, or meetings

Being held in high regard for work contributions.

References to being underpaid or wanting to increase pay.
The process, motivations, usage, and intent behind NTT evaluations

Evaluations that mention expectations beyond teaching

Remediation plans or improvement plans as a result of negative evaluations

Information that was viewed as helpful to improve teaching provided through the evaluation process

References to informal evaluations through observing performance unofficially on a day-to-day basis.

Information on how evaluations are administered. This is typically compliance driven

Statements of confusion, concern, or uncertainty about how evaluations are used to determine contracts and retention.

The feeling that NTT are not expected to do much because of their lowly status

Statements that the evaluations aren't being used to make decisions

NTT have status and their contracts roll over. Mentions of evaluations having no impact once the security of status is reached 


\section{Name}

Student Feedback

There is not really substance

there

They don't know what I do

Identity

Alumni

Don't tell

Faculty Governance

I didn't really have to go

Input not wanted

Invited to meetings

You're not invited

Hierarchy

I don't see status

There is a power dynamic

I love it here, but

Just NTT

Labor Union

\section{Description}

Student feedback on teaching formally in evaluations or informal from student comments

Comments about not getting specific feedback that is wanted to improve or inform teaching. Never hearing anything back or perfunctory.

Feeling that the person evaluating lacks the skill to provide feedback

Tacit assumptions, social norms, inclusion in workplace, hierarchy, espoused beliefs, values, that impact the NTT experience on identity

Connections to campus through past experience being a student at this institution

Voiced concern that information provided in the interview could somehow be linked back to the participant. Concerns for confidentiality. This is tied with fear of job loss, lack of security and stability.

Inclusion in meetings and committees

NTT were informed of meetings but not required or encouraged to participate

Inclusion by physical integration only not full participation in decision making. Lacking social integration and/or input in faculty governance.

Statements of physical inclusion in faculty meetings and governance.

NTT feeling excluded from departmental, college or university meetings

Mentions of the tiered system ranking $\pi T$ faculty above NTT

Statements that all people are equal regardless of classification.

NTT feeling of not having input or influence on matters of importance. Statements of a division or hierarchy between NTT and TT faculty

Statements of pleasure and displeasure of the workplace. Vacillating or mixed emotions.

statements to minimize or devalue the position of NTT as less than. Often self-deprecating

Mentions of the NTT labor union 
Role of NTT

NTT Types

Aspiring Academic

Career Ender

Freelancer

Specialist

Essential Specialists

Tacit Assumptions

That's not for you

\section{NTT Network}

Formal supports

Assigned Support colleagues

Orientation

learn as you go

I have a friend

It's chemistry

It's NTT teaching NTT
What is the role of the NTT on campus? Main purpose or function. Why the university has NTT employees

Based on Leslie and Gappa categories

These NTT may hold terminal degrees and may teach courses at multiple institutions to piece together a wageearning job.

NTT faculty who are retired and teaching courses to fill in on a temporary basis. These NTT do not intend on working fulltime.

NTT faculty that chooses to teach part-time for the benefit of having a flexible schedule.

NTT with discipline specific expert knowledge. These individuals may teach full-time or have a career in a related profession and teach contingently.

Specialist that holds additional credentials beyond specialty degree. For example, certifications.

implied or assumed norms based on core beliefs and values

exclusion by statements or artifacts

Informal sources of support that were sought from friends, past connections, or other NTT

University designed supports through orientations or assigned colleagues responsibilities

Program directors, teaching leads or others assigned as support roles. Individuals viewed as more experienced with formal responsibility in supporting NTT. Explicit relationship defined.

orientation to the department, university, onboarding process to prepare NTT for their jobs

statements of NTT figuring out procedural information through on-the-job observation, trial, and error

Having previous connections to the department. Possibly alumni status or close friends

NTT connecting with one another from an affinity or common bond

Having felt 'lost' themselves as new NTT a network is formed to welcome and orient new NTT. This is informal and not a required part of the job. 


\section{Name}

Procedural Knowledge

administrator or clerical

assistant

email

Seek it out or be lost

So, where my office was located.

\section{Description}

Nuts and bolts within a department. i.e., copies, text selection, departmental policies, office hours.

Individuals who answer questions about procedural items

emails to communicate procedural information such as syllabi, copies, parking etc.

Acclimating to the environment through observing others' behaviors, asking questions or independently exploring sources of information
So, where my office was located. NT
Preparation at hire
Confident previous work
experiences

Nervous

Feelings of nervousness. NTT was prepared but nervous

Not prepared

People who did not feel prepared to teach

Participant in my own education

References to having been a student or teaching in the way they were taught. Modeling behaviors

Taught as a GA Having experience teaching as a graduate assistant

Professional Development

Formal training

Ends

References to Professional development for its own sake

Formal Mentor Program

University or Dept. Designed mentor program for support in pedagogy

funding for PD

monies made available to pursue professional development.

Learn and connect to the culture

Means

References to meeting others, connecting to one another, the culture of the university or belonging as a result of PD references to PD as a method or way to some other end. Possibly better evaluations or being hired to teach. 